6,5668630

\title{
Coordinated Location-based Downlink Scheduling in a Cellular CDMA Network with Partitioned Cells
}

by

\section{GANG WU}

(MEng, Beijing University of Aeronautics and Astronautics, China, 1995) (BSc, Beijing University of Aeronautics and Astronautics, China, 1991)

A thesis

presented to Ryerson University

in partial fulfillment of the

requirement for the degree of

Master of Applied Science

in the Program of

PADPERTY OF

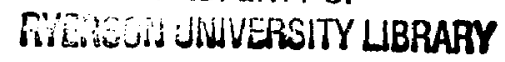

Electrical and Computer Engineering

Toronto, Ontario, Canada, 2004

(C)ANG WU 2004 


\section{UMI Number: EC53018}

All rights reserved

INFORMATION TO USERS

The quality of this reproduction is dependent upon the quality of the copy submitted. Broken or indistinct print, colored or poor quality illustrations and photographs, print bleed-through, substandard margins, and improper alignment can adversely affect reproduction.

In the unlikely event that the author did not send a complete manuscript and there are missing pages, these will be noted. Also, if unauthorized copyright material had to be removed, a note will indicate the deletion.

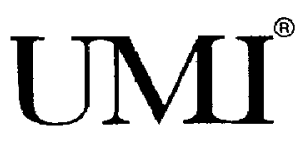

UMI Microform EC53018

Copyright 2008 by ProQuest LLC

All rights reserved. This microform edition is protected against unauthorized copying under Title 17, United States Code.

ProQuest LLC

789 East Eisenhower Parkway

P.O. Box 1346

Ann Arbor, MI 48106-1346 


\section{AUTHOR'S DECLARATION}

I hereby declare that I am the sole author of this thesis.

I authorize Ryerson University to lend this thesis to other institutions or individuals for the purpose of scholarly research.

Signature:

I further authorize Ryerson University to reproduce this thesis by photocopy or by other means, in total or in part, at the request of other institutions or individuals for the purpose of scholarly research.

Signature 


\section{Borrower's Page}

Ryerson University requires the signatures of all persons using or photocopying this thesis. Please sign below, and give address and date.

\begin{tabular}{|c|c|c|c|}
\hline Name & Signature & Address & Date \\
\hline & & & \\
\hline & & & \\
\hline & & & \\
\hline & & & \\
\hline & & & \\
\hline & & & \\
\hline & & & \\
\hline & & & \\
\hline & & & \\
\hline & & & \\
\hline & & & \\
\hline & & & \\
\hline & & & \\
\hline & & & \\
\hline & & & \\
\hline & & & \\
\hline & & & \\
\hline & & & \\
\hline & & & \\
\hline & & & \\
\hline & & & \\
\hline & & & \\
\hline & & & \\
\hline & & & \\
\hline & & & \\
\hline & & & \\
\hline & & & \\
\hline
\end{tabular}




\title{
Coordinated Location-based Downlink Scheduling in a Cellular CDMA Network with Partitioned Cells
}

\author{
Master of Applied Science 2004 \\ Gang Wu \\ Electrical and Computer Engineering \\ Ryerson University
}

Abstract

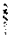

A downlink scheduling scheme, called coordinated location dependent downlink scheduling scheme (CLDSS), that combines the intra-cell power allocation and inter-cell transmission coordination is proposed to be used in TD-CDMA networks. In the proposed scheme, each cell in the cellular network is partitioned into co-centric areas based on the load

- distribution in the cell. The transmissions from base stations are controlled based on the intra-cell load as well as coordinated to minimize inter-cell interference.

The average throughput employing the CLDSS is analyzed for a 2-(square) cell system in shadow fading environment with 2 (square) partitioned areas for each cell. Simulation study is also done to validate the numerical results obtained from the analytical study. It is shown that CLDSS scheme can provide soft throughput, i.e., the average throughput remains relatively invariant with the number of users and also provide good performance even in the non-uniform user distribution within a cell. The CLDSS scheme can also improve the fairness in terms of achievable throughput to users anywhere in the cell. 


\section{Acknowledgment}

I appreciate the support and encouragement from Dr. Alagan Anpalagan, my supervisor. His expertise in wireless communications is very helpful for this work.

I would like to thank all the committee members for their participation in my thesis defense.

I would like to thank the Electrical and Computer Engineering Department at Ryerson University for the financial support of my study and research.

I would like to thank all staff of the Electrical and Computer Engineering at Ryerson University for their kind help.

I wish to convey the warmest thanks to my family for their endless support. 


\section{Contents}

1 Introduction 1

2 Characteristics of TDD-CDMA Systems $\quad \mathbf{8}$

2.1 Elements of a Wireless Communication System . . . . . . . . . . 9

2.2 Features of TDD Transmission $\ldots \ldots \ldots \ldots \ldots$

2.3 Downlink of a CDMA System . . . . . . . . . . . . . . 11

2.4 System Model of TDD-CDMA . . . . . . . . . . . . . . 13

2.5 Summary . . . . . . . . . . . . . . . . . . 14

3 Previous Scheduling Schemes in a HDR-like Wireless Communication $\begin{array}{ll}\text { System } & 16\end{array}$

3.1 Algorithm 1: Best Channel First (BCF) . . . . . . . . . . . . 16

3.2 Algorithm 2: HDR-as proposed originally by Qualcomm . . . . . . . . 17

3.3 Algorithm 3: Hybrid Automatic Repeat Request (H-ARQ) in the HDR System . . . . . . . . . . . . . . . . . . . . 18

3.4 Algorithm 4: Power Allocation Scheme in the HDR System . . . . . . . 19

3.5 Algorithm 5: Inter-Cell Coordination Schemes . . . . . . . . . . . . . . 21

3.6 Algorithm 6: A Coordination Downlink Scheduling in Linear Data-only Networks . . . . . . . . . . . . . . . . . . . . . . 22

3.7 Algorithm 7: Dynamic Channel-Sensitive Scheduling Algorithm . . . . . . 24

3.8 Other Scheduling Algorithms _. . . . . . . . . . . . . 2 25

3.9 Summary . . . . . . . . . . . . . . . . . . 26

4 Coordinated Location-based Downlink Scheduling Scheme (CLDSS) with Partitioned Cells $\quad 27$

4.1 System Model Description . . . . . . . . . . . . . . . . . . . . . 29

4.2 Intra Cell Scheduling: HDR-like Scheduling . . . . . . . . . . . . . . 31

4.3 Inter Cell Scheduling: Proposed Scheduling Scheme (CLDSS) . . . . . . . . 33

4.4 Summary . . . . . . . . . . . . . . . . . . . . 35

$5 \quad$ Analysis of the CLDSS $\quad 37$

5.1 Analysis of Average Throughput of a System with the CLDSS . . . . . . 37

5.2 Performance Evaluation - Numerical Results . . . . . . . . . . . . . . . 42 
5.2.1 Results with Uniform User Distribution . . . . . . . . . . . . 45

5.2 .2 Results for Non-Uniform User Distribution . . . . . . . . . . . . 47

5.2 .3 Effect of User Density . . . . . . . . . . . . . . . . 49

5.2 .4 Modification of the Proposed Scheme . . . . . . . . . . . 53

5.2 .5 Effect of the Number of Users in the System . . . . . . . . . . 56

5.3 Summary ............................... 58

6 Simulation and Results $\quad 59$

6.1 Comparison of the CLDSS I Scheme with the ULISS scheme . . . . . . 63

6.1.1 Effect of the Normalized Distance on the Throughput of System with Uniform User Distribution ... . . . . . . . . . . . . . 63

6.1.2 Effect of the Normalized Distance on the Throughput of System with Non-Uniform User Distribution . . . . . . . . . . . 65

6.1.3 Effect of the User Density on the Throughput . . . . . . . . . 66

6.1 .4 Modification of the CLDSS . . . . . . . . . . . . . 66 66

6.1 .5 Effect of the Number of Users on the Throughput . . . . . . . . 67

6.2 Comparison of CLDSS I Scheme with CLDSS II Scheme . . . . . . . . 67

6.2.1 Results with Uniform User Distribution . . . . . . . . . . . . . 69

6.2.2 Results for Non-Uniform User Distribution . . . . . . . . . . . . 70

6.2.3 Effect of User Density on the Throughput . . . . . . . . . . 71

6.2.4 Effect of User Number on the Throughput . . . . . . . . . 73

6.3 Summary ........................... 73

$\begin{array}{lll}7 & \text { Summary } & \mathbf{7 5}\end{array}$

7.1 Conclusion . . . . . . . . . . . . . . . . 75

7.2 Future Work . . . . . . . . . . . . . . . . . 78 


\section{List of Figures}

1.1 A Cellular System. . . . . . . . . . . . . . . . . . 2

1.2 TDMA Frame Structure. . . . . . . . . . . . . . . . . 4

1.3 3-D Resources in CDMA Scheme. . . . . . . . . . . . . . . 4

1.4 Various Upgrade Paths for $2 \mathrm{G}$ Technologies $[1] \ldots \ldots \ldots$

2.1 A Wireless Communication System. . . . . . . . . . . . . . 9

2.2 The TDD Transmission. . . . . . . . . . . . . . . . . 10

2.3 Signal and Interference Power before and after Despreading in a CDMA System. . . . . . . . . . . . . . . . . . . . 12

2.4 Downlink Allocation Geometry. . . . . . . . . . . . . . . . 13

2.5 TDD-CDMA Scheme. . . . . . . . . . . . . . . . . 14

3.1 Frame Format in Time Coordinated Scheduling. . . . . . . . . . . . 21

3.2 ON/OFF States of Cell $E_{0}$ 's Neighboring Base Stations during its L-time, R-time, 0 -time and 2 -time. . . . . . . . . . . . . 23

4.1 TD-CDMA Frame Structure. . . . . . . . . . . . . . . . . . . . 29

4.2 A Two-cell System Model. . . . . . . . . . . . . . . . . . . . . . 30

4.3 Scheduling and Power Allocation in CLDSS. . . . . . . . . . . . . 35

5.1 The Density Function of User Distribution in a Cell. . . . . . . . . . . . 38

5.2 Curve-fitting of Average Throughput of CLDSS in a 2-cell System with Normalized Distance $d^{*}$. . . . . . . . . . . . . . . . . . . 42

5.3 Comparison of ULISS, CLISS and CLDSS Schemes. . . . . . . . . . 44

5.4 Throughput with Uniform User Distribution. . . . . . . . . . . . . . . . 45

5.5 Throughput with the Non-uniform Distribution of Users. . . . . . . . . . 48

5.6 Relationship between Key Point and the User Density of the Inner Area . . 50

5.7 Throughput Affected by User Density. . . . . . . . . . . . . . 51

5.8 Throughput Affected by User Percentage. . . . . . . . . . . . . . . 52

5.9 Throughput of Modified CLDSS. . . . . . . . . . . . . . . 54

5.10 Throughput Impacted by Fairness Adjustment Parameter $(K)$. . . . . . 55

5.11 Throughput Affected by the Number of Users $(N) \ldots \ldots \ldots 7$

6.1 Parameters Used in the Simulation. . . . . . . . . . . . . 60 
6.2 Flowchart of the Procedure in the Simulation. . . . . . . . . . . . . 62

6.3 Throughput Affected by the Normalized Distance $\left(d^{*}\right)$ with Uniform User Distribution. . . . . . . . . . . . . . . . . . . . . 64

6.4 Throughput Affected by the Normalized Distance with Uniform User Distribution. $0.3<d^{*}<0.8 \ldots \ldots \ldots \ldots$. . . . . . . . . . 64

6.5 Throughput with Non-uniform User Distribution. . . . . . . . . . . . 65

6.6 Throughput Affected by User Density. . . . . . . . . . . . . . . . . 67

6.7 Throughput Affected by User Percentage. . . . . . . . . . . . . . . . . . 68

6.8 Throughput Affected by the Number of the Users in a Cell. . . . . . . . . . 68

6.9 Throughput with Uniform User Distribution. . . . . . . . . . . . . . . 69

6.10 Throughput with Non-uniform User Distribution. . . . . . . . . . . . 71

6.11 The Effect of User Density on the Throughput. . . . . . . . . . . . . . 72

6.12 The Effect of User Number on the Throughput with the Non-uniform User Distribution. . . . . . . . . . . . . . . . . 72

6.13 The Relationship between the Key Points, Normalized Distance $\left(d^{*}\right)$ and the Density of Users in the Inner Area $\left(\rho_{a}\right) \ldots \ldots . \ldots 74$

7.1 The Model for the Future Work. . . . . . . . . . . . . . . . 78 


\section{Chapter 1}

\section{Introduction}

TN recent years, high data rate (HDR) wireless data service has become increasingly velopment of the Internet and rapid growth and nearly universal coverage of wireless communication, the requirement to increase the transmission rate of wireless data service continues to rise.

Internet services almost exist every where. People can get the news, documents, music, movie, game and other kinds of information via Internet anytime. Internet also works as a very efficient tool for the individuals in business and the professionals. Currently, the wireline communication system can provide high speed Internet service which can normally meet the requirement of users, such as digital subscriber line and cable can provide the Internet service with data rates above $2 \mathrm{Mbps}$. Meanwhile, the subscribers of a wireless communication system also want to access the Internet with high data rate. When users absent from their office (in transit or professional meetings), it is essential for them to retrieve e-mail or report, order airline tickets, browse web and so on through the Internet. Generally, they demand the same data service available in their home base. Hence, how to improve the data rate of wireless communication system is a key topic.

For the purpose of understanding the concepts and the developing trends of wireless communication, we recall a brief history of the evolution of wireless communication. The cellular concept was first developed in 1970s. Since 1990, the cellular communication 
industry has grown explosively. In a cellular system (see Fig. 1.1), there are many low power transmitters each of them covers a small portion of the service area - which is called "cell". Each base station in a cell is assigned a portion of the total channels allocated to the entire system. The neighbor base stations can be allocated different groups of channels in order to reduce the co-channel interference between the base stations and the mobile users. When two cells are separated from each other and the distance is large enough to keep the interference levels within tolerable limits, the base station in the two cells can be allocated the same group of channels, this concept is called "frequency reuse". By means of frequency reuse, we can improve the capacity of the cellular system. In Fig. 1.1, A, $\mathrm{B}, \mathrm{C}, \mathrm{D}, \mathrm{E}, \mathrm{F}, \mathrm{G}$ represent the groups of channels allocated to the cells respectively. If two cells have the same letter, it means that the two cells are allocated the same group of channels, which are commonly called "co-channel" cells .

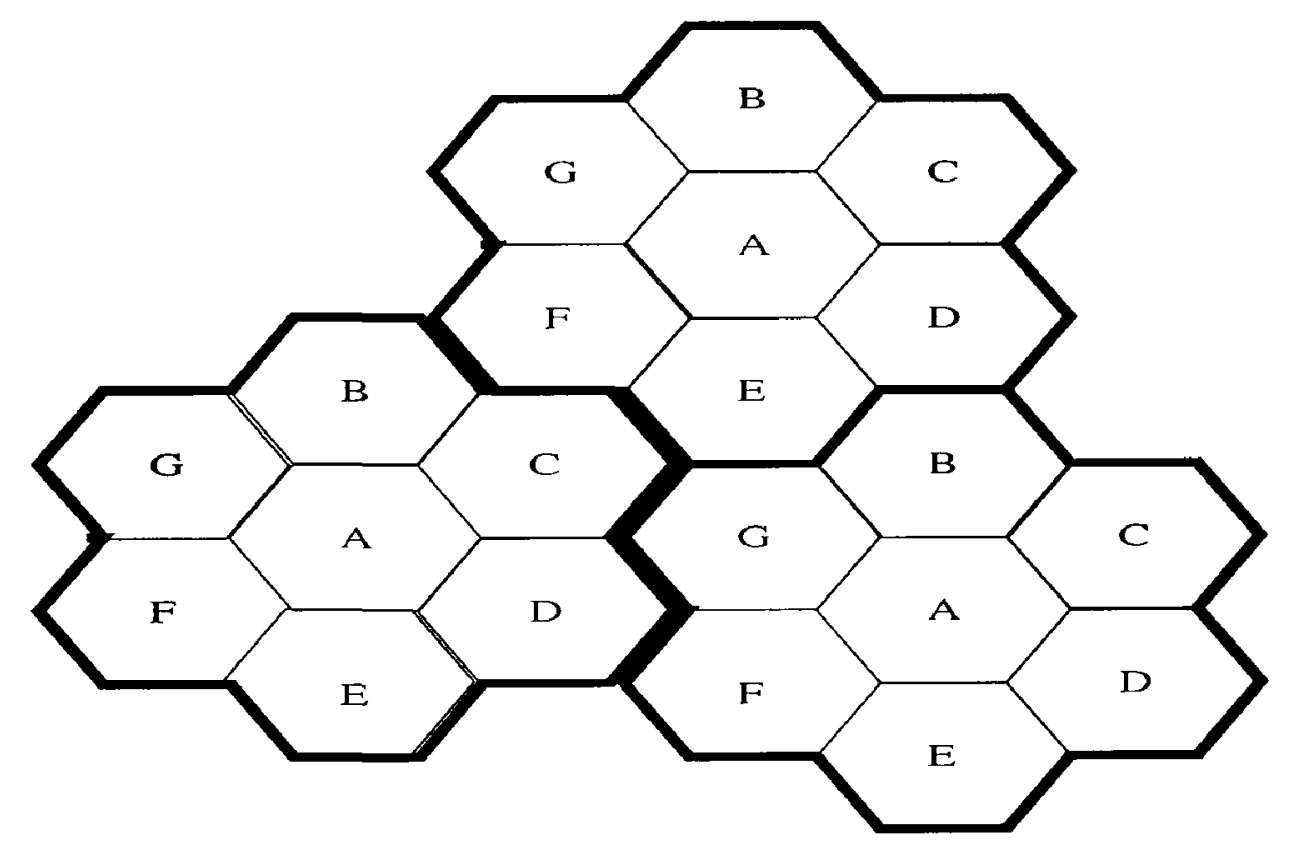

Figure 1.1: A Cellular System.

To support multiple access, there are several techniques in use for wireless communications. Frequency Division Multiple Access (FDMA), Time Division Multiple Access (TDMA) and Code Division Multiple Access (CDMA) are the major and popular schemes [1]. 
- FDMA is the division of the frequency band allocated for wireless cellular communication into many channels, each of which can carry a voice conversation or, with digital service, carry digital data. FDMA is a basic technology in the analog Advanced Mobile Phone Service (AMPS), the most widely installed cellular telephone system in North America. With FDMA, each channel can be assigned to only one user at a time in a cell.

- TDMA is digital transmission technology that allows a number of users to access a single radio-frequency $(\mathrm{RF})$ channel without interference by the allocation of unique time slots to each user within each channel. The current TDMA standard for cellular divides a single channel into six time slots, with each signal (or user) using two slots, providing up to 3-fold capacity gain over advanced mobile-phone service (AMPS). TDMA system uses a buffer-and-burst method to transmit data to the mobile users, as a result, the transmission is non-continuous. The TDMA frame structure is shown as Fig. 1.2, where a frame is comprised of $\mathrm{N}$ time slots. The frame is cyclically repeated over time. The users in TDMA system interlace the transmission with each other.

- CDMA is a digital cellular technology that uses spread-spectrum techniques. Unlike competing systems, such as "Global System for Mobile Communication" (GSM), that use TDMA, CDMA does not assign a specific frequency to each user. Instead, every channel uses the full available spectrum. Individual conversations are encoded with a pseudo-random digital sequence. Each channel in CDMA system is assigned a unique pseudo-noise (PN) code which is (approximately) orthogonal to PN codes used by other users as shown in Fig. 1.3.

Today, some worldwide standards have been developed. If we focus on the current cellular networks which are commonly called second generation or $2 \mathrm{G}$ technologies, we can note that the most popular standards include three TDMA standards and one CDMA standard: (1) Global System Mobile (GSM), (2) Interim Standard 136 (IS-136), (3) Pacific 


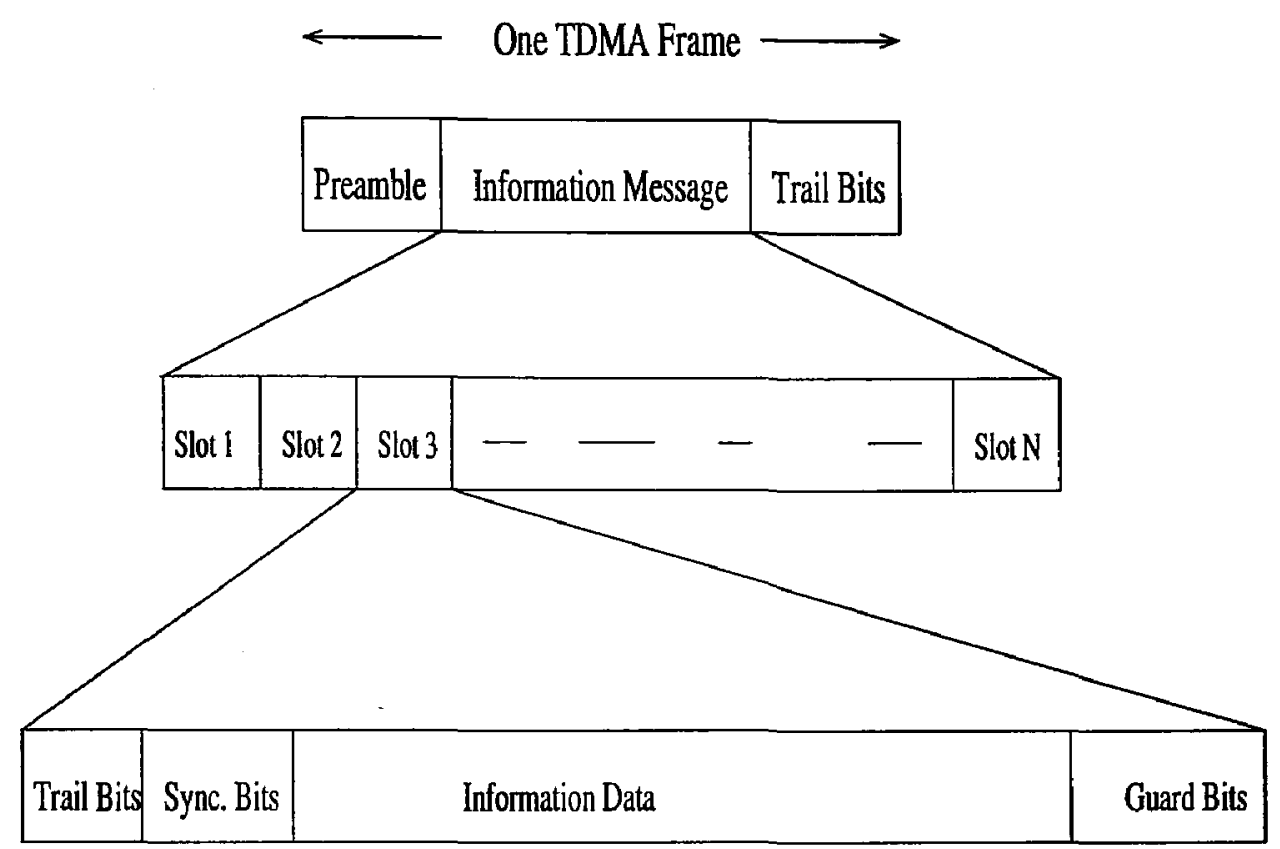

Figure 1.2: TDMA Frame Structure.

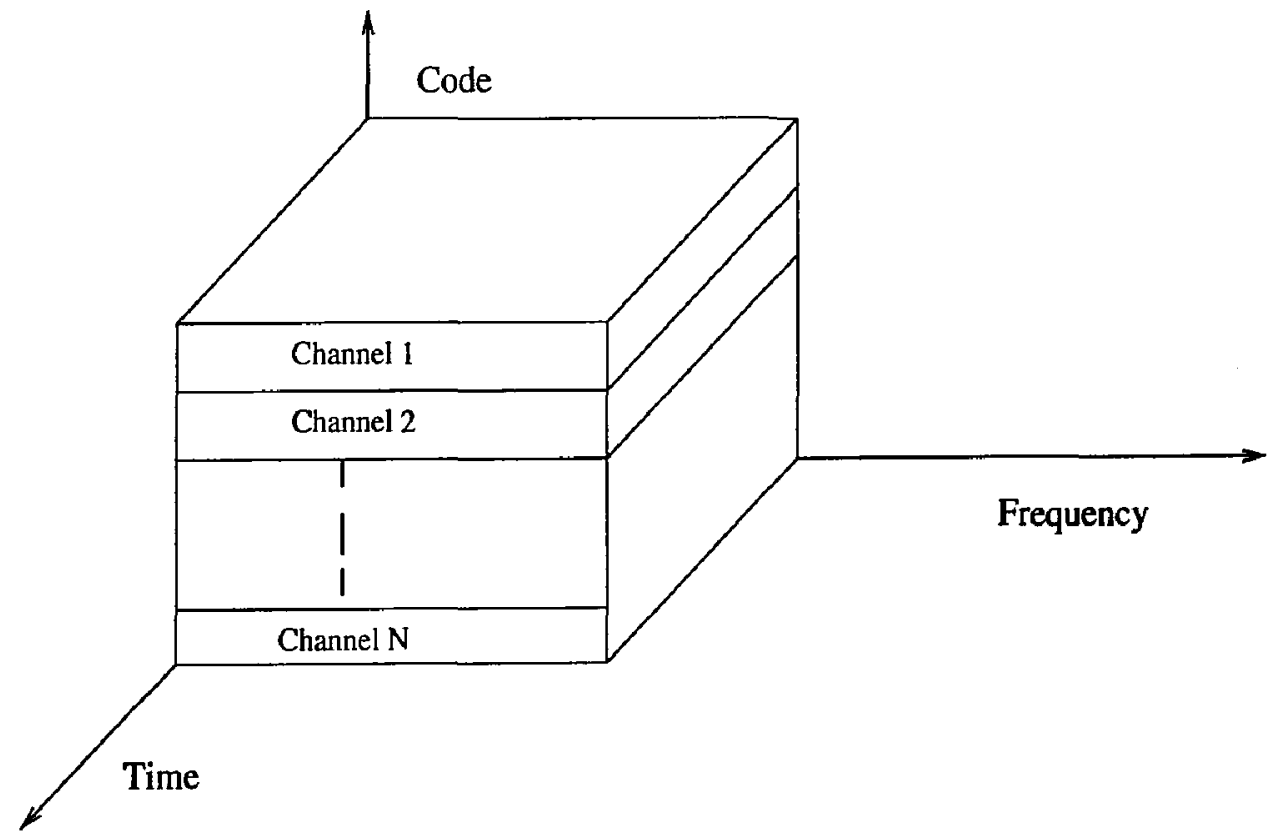

Figure 1.3: 3-D Resources in CDMA Scheme. 
Digital Cellular (PDC), (4) The popular 2G CDMA standard Interim Standard 95 Code Division Multiple Access (IS-95), also know as cdmaOne.

Because of the advantages of CDMA which will be discussed in detail in the next chapter, CDMA is widely deployed by carriers in North America. With the widespread use of Internet, the $2 \mathrm{G}$ standards are modified or retrofit for compatibility; as a result, the 2.5G (IS-95B) and 3G (CDMA2000) standards are proposed as shown in Fig. 1.4.

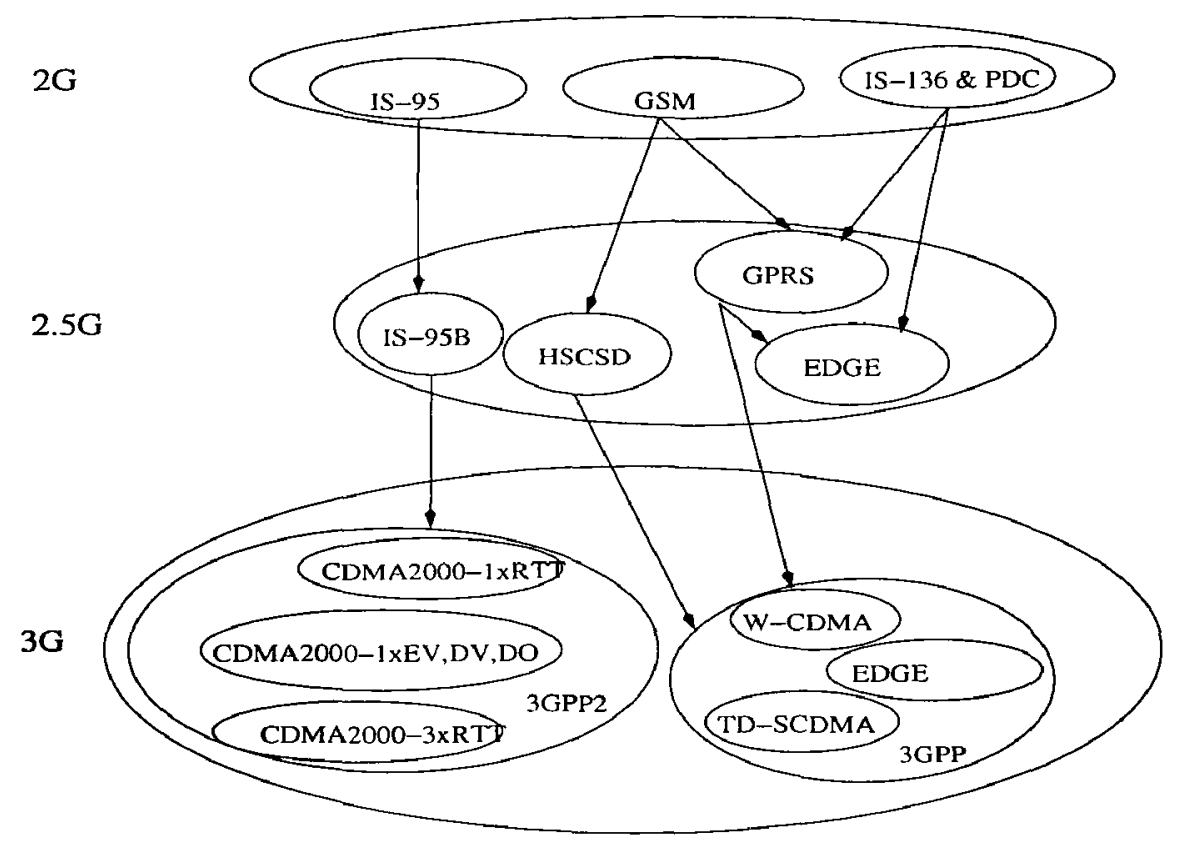

Figure 1.4: Various Upgrade Paths for 2G Technologies [1].

3G systems promise several advantages, such as, multi-magabit Internet access, communication using Voice over Internet Protocol (VolP), increased network capacity [2]. In the 3G wireless communication system, the characteristics of high speed data communication are fundamentally different from voice communication, including traffic asymmetry and tolerance to latency. For high speed data communication, such as downloading from the Internet, the throughput demanded by downlink (transmission from base station to mobile users) is likely to be several times greater than the uplink (transmission from mobile users to base station). In the meantime, two-way conversational speech requires strict adherence to symmetry.

On the other hand, interference suppression is very critical to increase the throughput 
of the system based on CDMA technology.

Based on these new features of 3G systems, we propose a scheduling scheme - Coordinated Location-based Downlink Scheduling Scheme (CLDSS) Partitioned Cells. The motivation of this proposed scheme is to improve the downlink throughput of the system by reducing the interference coming from neighbor cells. In general, when a user is located near the border of a cell, the user is far away from the home base station, therefore the base station has to transmit signal with high power. But in a CDMA system, this power also produces co-channel interference to the neighbor cell's users; especially, this affects the users located near this border. The proposed scheme coordinates the transmission of different cells based on the location of the mobile users. We partition a cell into flexible co-centric areas and allocate time slots to the mobile users based on the distribution of the users in the two areas. We assign different levels of radio signal power to the users based on their location. If the distance between the user and base station is short, the base station transmits data signal with low power; otherwise, the radio signal power is high. In the meantime, the cells coordinate their transmissions with each other.

The contribution of the thesis is that: in this research work,

1. We propose a downlink scheduling scheme, called coordinated location dependent downlink scheduling scheme (CLDSS), that combined the intra-cell power allocation and inter-cell transmission coordination proposed to be used in TD-CDMA networks.

2. We analyze the average throughput employing the CLDSS for a 2-cell system in shadow fading environment with 2 partitioned areas for each cell.

3. We perform a simulation study to validate the numerical results obtained from the analytical study.

4. We show that the proposed scheme can (a) provide soft throughput, (b) perform better even with the non-uniform distribution of users, (c) improve the fairness in terms of achievable throughput to users anywhere in the cell. 
5. We compute the appropriate size of the partitioned areas by analysis and also confirmed by the simulation - useful in the design of the network.

The thesis is organized in the following way. Next chapter introduces the characteristic of a TD-CDMA system. Chapter 3 discusses previous scheduling schemes in HDR-like system. We propose a new scheduling scheme (CLDSS) for downlink of a CDMA system in Chapter 4. Chapter 5 analyzes the performance of the CLDSS scheme. In chapter 6 , we provide the results of the simulation studies based on the proposed scheme. We summarize the thesis in Chapter 7 with the conclusion of the thesis and future work. 


\section{Chapter 2}

\section{Characteristics of TDD-CDMA Systems}

$7 \mathrm{HE}$ TDD-CDMA technique is a time division duple (TDD) mode of code division

1 multiple access (CDMA) technology for wireless communications [3]. It is the combination of the TDMA technology and CDMA technology. One of the motivation for using the TDD-CDMA technology is to take advantage of the time slots of the TDMA. We can involve some scheduling algorithms in the time slots in order to control the data transmission. The other motivation is to take advantage of the characteristics of the CDMA in order to limit interference from other users and other base stations. The TDD-CDMA technology can be used to improve the performance of the wireless communications for the high speed data services. The TDD-CDMA technology has been employed in $3 \mathrm{G}$ systems. For instance, two TDD-CDMA standards have already been adopted in the standards of 3G communication: (1) time-division/code-division multiple access (TD-CDMA) is part of the 3GPP standards [2] (see Fig. 1.4) and (2) the time-division/synchronous codedivision multiple access (TD-SCDMA) [2]. For the purpose of clearly understanding the characteristics and the advantages of the TDD-CDMA, we introduce some related concepts next. 


\subsection{Elements of a Wireless Communication System}

When we design and analyze a wireless communication system, one of the most important and difficult parts is the characteristics of the physical channels through which the signals are transmitted. In the wireless communication, not only the channels change with the movement of the mobile users, but also they are effected by the environment which includes path loss, multi-path and fading effects. At present, some technologies have been developed to mitigate these effects, i.e., power control, exploiting multi-path signal arrival and antenna diversity which are shown in blocks in Fig. 2.1 [3] [4].

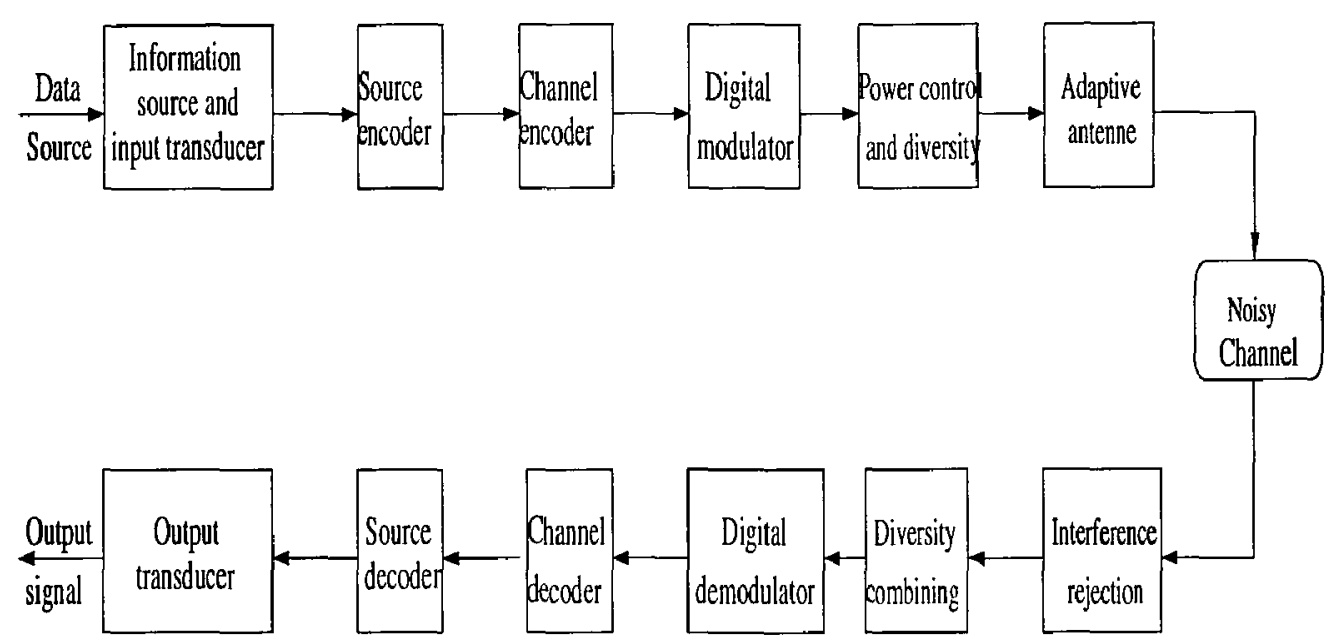

Figure 2.1: A Wireless Communication System.

The information source may be voice or video which can be converted into a digital stream by a source encoder, or may be a data stream, such as a TCP/IP source. Then, the source is modulated in a CDMA system-the narrow signal bandwidth is spread over a large frequency spectrum. Mobile communication channels are generally characterized by multi-path signal arrival and fading. Power control (or allocation) and transmission diversity are necessary in order to compensate for the effects of channel fading. The TDDCDMA technology can play a very important role in the power control (or allocation), diversity transmission (and reception), and adaptive antenna functions. 


\subsection{Features of TDD Transmission}

There are several methods to achieve the duplex transmission of information between users and base stations. The most common methods are FDD and TDD transmission. FDD technology uses separate frequency channels for duplex transmission (uplink and downlink directions). TDD technology uses the same frequency band for transmitting and receiving alternately as shown in Fig. 2.2.

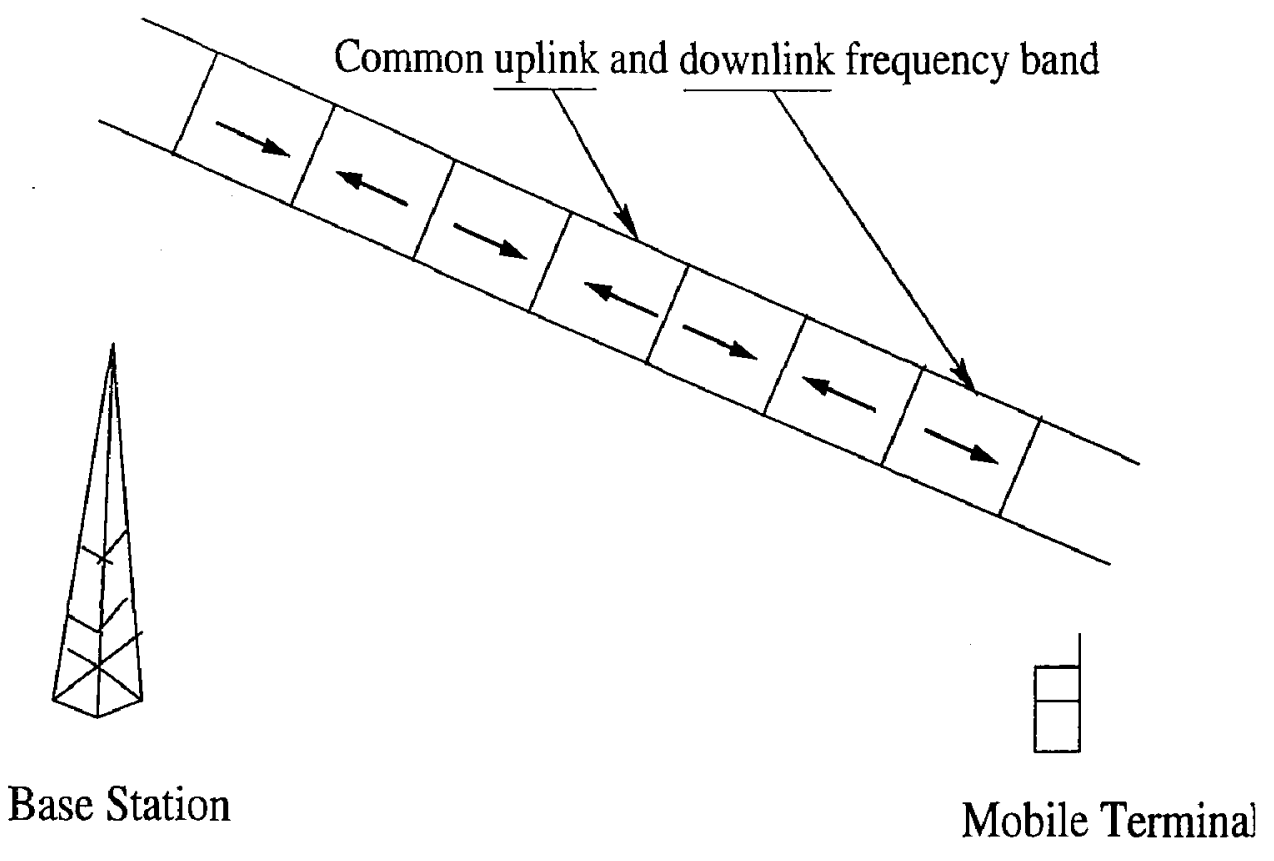

Figure 2.2: The TDD Transmission.

In a TDD system, uplink and downlink transmit in the same frequency band. The TDD system accomplishes the duplex transmission by ways of allocating certain time slots for each transmission and switching alternately by the uplink and downlink. The lengths of time slots can be unequal and the number of the time slots for the uplink and downlink can be flexible which are based on the requirements of users. For example, because of traffic asymmetry of Internet service, we can allocate most of the time slots for the downlink transmission in order to improve the transmission rate. In a multi-user system, we can assign different time slots to different users which realize the multiple access services. As well, we can allocate more time slots to a certain user to improve the 
performance based on the channel condition or the service priority. On the other hand, based the characteristics of the high speed data services (discussed in the next chapter), the communication system demands the base station to transmit data information to one user at a time, and the system has the capacity to provide multiple access. The TDD technology gives us the opportunity to involve some scheduling schemes to achieve this goal.

There are some advantages of TDD technology which make the performance of TDD to be better than FDD technology:

- TDD technology can efficiently utilize bandwidth. Since the uplink and downlink transmission share the same frequency bandwidth in a TDD system, TDD technology can be realized in non-paired and continuous bands.

- Since both uplink and downlink transmission use the same frequency channel in TDD system, the channel characteristics are reciprocal for the two direction. The channel reciprocity is very important, which can be used for power control (or allocation) and transmission diversity responding to the time-varying mobile channel.

- The design of TDD system is simple. Since there is only one set of electronics (i.e filters, oscillators) required at both mobile users and base stations for both uplink and downlink transmission.

\subsection{Downlink of a CDMA System}

In a 'CDMA system, as discussed in the previous chapter, the narrow-band information signal is multiplied by a very large bandwidth signal called the spreading signal. All users share the same frequency bandwidth and can transmit simultaneously. Each user is assigned a unique code (pseudo-random codeword) to encode the information signal. The receiver knowing the code sequences of the user can decode the received signal after reception and recover the original data (as shown in Fig. 2.3). 

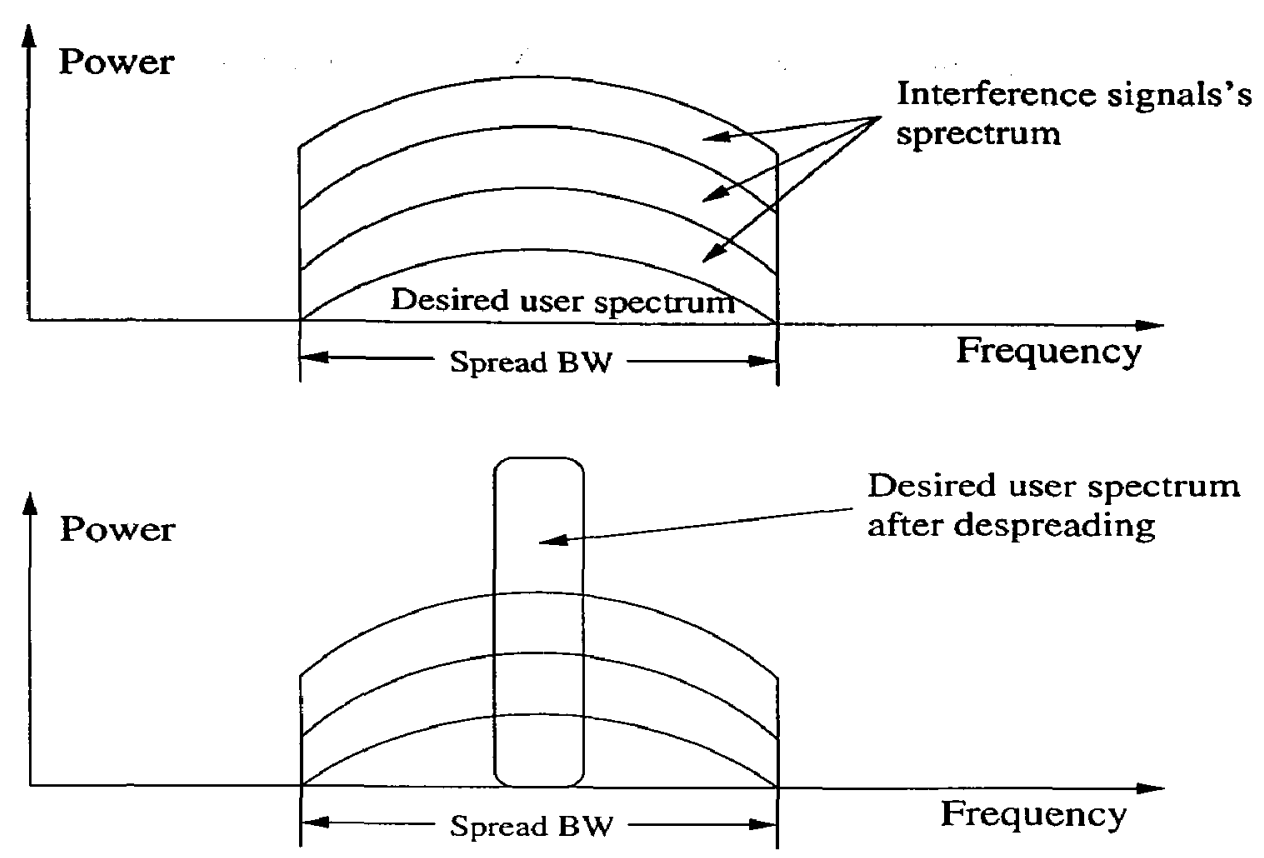

Figure 2.3: Signal and Interference Power before and after Despreading in a CDMA System.

The CDMA technology offer exclusive advantages, which made CDMA to be quite successful as a second-generation cellular system and adopted by the $3 \mathrm{G}$ standards, such as cdma2000 and WCDMA (as shown in Fig. 1.4). These advantages include:

- Many users can share the same frequency bandwidth in a CDMA system; either TDD or FDD technology can be used.

- CDMA has a soft capacity limit which means there is no absolute limit on the number of users in a CDMA system. But, the performance of the system degrades gradually as the number of users is increased.

- CDMA can reject interference. Cross-correlating the code signal with a narrowband signal will spread the power of the narrow-band signal thereby reducing the interfering power in the information bandwidth (see Fig. 2.3).

Since the transmissions in a high speed data application are asymmetric for uplink and downlink transmission. Today, in a non-real time data communication system (i.e downloading files from a wireless Internet network), the downlink transmission demands 
more throughput than the uplink transmission (i.e, uploading some information to a server). How to improve the performance of the downlink transmission is one of the hottest topics in $3 \mathrm{G}$ wireless communication research.

Since CDMA uses co-channel cells, the downlink suffers interference from a small number of other high-power base stations shown in Fig. 2.4. For the downlink transmission, the power allocation replaces of the power control (power control is used for uplink transmission) in accordance with the needs of individual subscribers in the cell.

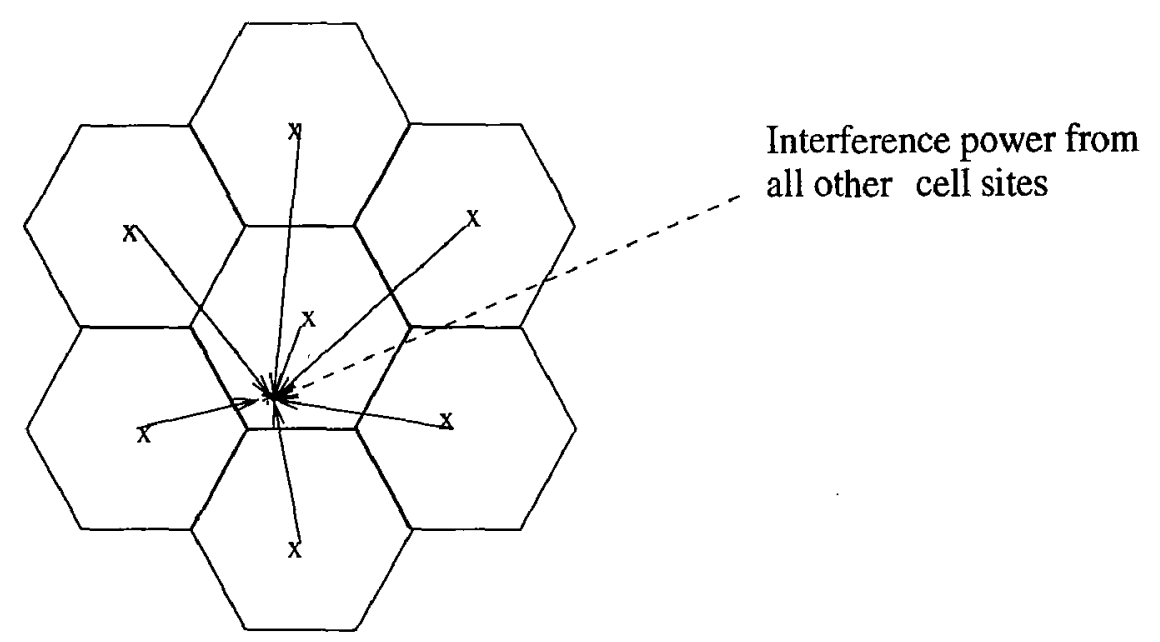

Figure 2.4: Downlink Allocation Geometry.

On the other hand, the transmission paths between the base stations and mobile users for the wireless communication can vary severely. The condition of paths can be affected by the environment, such as, buildings, mountains, and foliage. Hence, there are some statistical models to simulate the real world. The model considered in this thesis is based on the log-distance path loss and log-normal shadowing (discussed in detail in the next chapter).

\subsection{System Model of TDD-CDMA}

TDD-CDMA (also called TD-CDMA) combines TDMA and CDMA elements. In a TDDCDMA system, transmission and reception are confined and scheduled to a subset of all users in a cell at any particular time slot, which is as shown in Fig. 2.5. TDD- 
CDMA scheme inherits the advantages from both of TDMA and CDMA technology. This combined access method is shown to take advantage of the high intra-cell capacity of TDMA and the inter-cell interference suppression ability of CDMA. As well as, TDDCDMA can be used to improve the performance of high speed data communication by employing some special scheduling algorithms for data services as done in this thesis work.

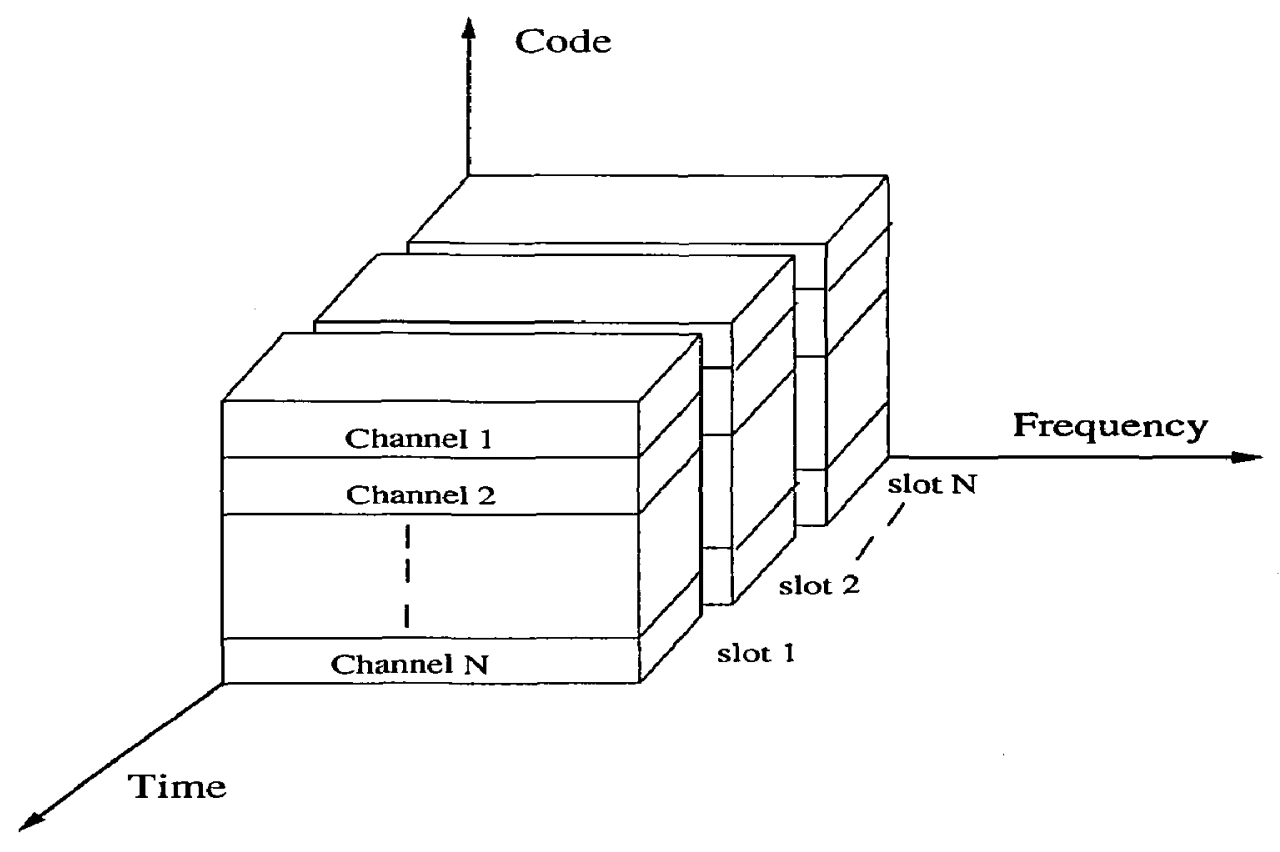

Figure 2.5: TDD-CDMA Scheme.

\subsection{Summary}

In this chapter, we introduced the basic concept of TDD-CDMA technology. TDD-CDMA technology is a CDMA technique with TDD mode, which has the advantages of both of TDMA and CDMA. For example, the TDD-CDMA technique can efficiently utilize bandwidth with accomplishing the duplex transmission of uplink and downlink in the same frequency band. TDD-CDMA can schedule the transmission and change the data rate by ways of allocating the certain time slots for the uplink and downlink transmission, as well as changing the lengths of the time slots. In the meantime, TDD-CDMA can reject interference and has a soft capacity. Because of the advantages of TDD-CDMA, 
the TDD-CDMA has already been adopted in the 3G communication standard. In the TDD-CDMA system, we can employ some scheduling algorithms in the time slots to improve the performance of high speed data communication system. 


\section{Chapter 3}

\section{Previous Scheduling Schemes in a HDR-like Wireless Communication System}

$\mathrm{P}$ RESENT generation wireless networks have been designed primarily for voice traffic, which are not suitable to support high data traffic. $3 \mathrm{G}$ standards are proposed to meet the increasing data traffic demand over mobile phone. In a data service like web browsing, web traffic is mainly on downlink and the uplink carries little traffic. For a system to support multiple access, within the limited transmission sources (i.e bandwidth, power, time slots), an efficient source allocation algorithm at the base station is required. Next, we discuss some of current HDR-like scheduling algorithms from the literature.

\subsection{Algorithm 1: Best Channel First (BCF)}

The Best Channel First (BCF) scheduling algorithm is a common approach to wireless packet scheduling [5]. Since channel conditions vary widely among users and are often uncorrelated, the limited transmission resources may be better utilized by serving users with better channel conditions who require less resource, i.e., transmit power. Instead of expanding a large amount of transmit power to serve a $M T$ with poor channel condition, the same amount of power can be used to serve more $M T s$ or transmit more data at a higher rate. A simple channel-dependent approach is to schedule and transmit packets in 
order of their channel conditions (best-channel-first). The BS selects MTs with the best channel conditions in each time slot and serves the packets destined for them first.

But, one obvious disadvantage of this scheme is that if an $M T$ is subject to long-term fading due to its location, for example, if a user is located near the border of the cells, its data may not be transmitted for an extended period of time.

\subsection{Algorithm 2: HDR-as proposed originally by Qual- comm}

The high-data-rate (HDR) [6] system is based on Internet protocols adapted to the mobile environment. The HDR scheduling algorithm was designed for the downlink transmission. Because of the vastly different requirements, high-data-rate users are separated from lowrate voice users by different RF carriers. The high-data-rate users are scheduled over time slots, where the slot lengths depend on channel conditions and transmission is executed with a constant maximum transmit power. In the HDR system, rate control replaces the power control [6] [7].

In the HDR scheme, in order to take advantage of the temporal variations of the channel, the system schedules transmission to a mobile user who has the strongest signal levels.

Before we introduce the algorithm in detail, we present some terms defined in the HDR algorithm. $D R C$ is the rate requested by the mobile user in a given time slot. $R$ is the average rate received by the mobile user over a window of appropriate size. $N$ is the number of the mobile user in the system. $R_{i}(t)$ is the estimate of the average rate for user $i$ at time slot $t, i=1, \ldots N$. Similarly, during the time slot $t, D R C_{i}(t)$ is the $D R C$ for user $i$. The aim for the algorithm is to send data to the mobile user that has the highest $D R C / R$. It works as follows:

- To estimate and transmit: The user with the highest ratio of $D R C_{i}(t) / R_{i}(t)$ out of all $N$ users will receive transmission at each decision time. 
- To update average rate regularly: The average rate for user $i$ is determined as:

$$
R_{i}(t+1)=\left(1-1 / t_{c}\right) R_{i}(t)+1 / t_{c} \times R_{c, i}
$$

where $R_{c, i}$ means current transmission rate of user $i, t_{c}$ is a time constant which is assumed to be 1000 time slots.

The HDR algorithm exploits time-varying channel conditions and improves overall throughput of a CDMA system. The HDR algorithm also satisfies fairness property known as proportional fairness [8], which is proved in [7]. That is, if another scheduling algorithm is used to increase the throughput of a specific user by $x \%$ over what that user receives under the HDR scheduling algorithm, the sum of all the percentage decreases suffered by the throughput of all the other users under the new algorithm will be more than $x \%$.

But, the HDR algorithm does not pay attention to the priority, delay bound of some certain mobile users. In the mean time, the base station always radios full signal power which increases the interference to the users in the neighboring cell.

Throughout the thesis, we refer to "HDR-like" scheduling algorithm as the one that is based on the original HDR schedme proposed by Qualcomm in [6] with the feature that, in any time slot, only a subset of users are scheduled for transmission based on some criteria, such as priority, channel condition or maximum achievable rate.

\subsection{Algorithm 3: Hybrid Automatic Repeat Request (H-ARQ) in the HDR System}

The hybrid automatic repeat request (H-ARQ) scheme combines error correction (implemented with turbo codes), error detection (implemented with CRC code), soft packet combining and the use of incremental redundancy [9]. H-ARQ scheme reduces the required downlink $S I R$ (signal-to-interference ratio) and thereby improves overall system capacity, particulary in mobile environments. 
In the original HDR system, a mobile user determines the $D R C$ from the prediction of the $S I R$ expected to be seen by the mobile user over the slot during which the transmission will occur. SIR predictions are made from current and past $S I R$ estimates. In order to achieve a small packet error rate (PER), DRC requests are conservative, especially in fast changing channel conditions. These conservative requests often result in excess $S I R$, which could have been used to achieve increased throughput. The H-ARQ scheme employs an acknowledgement bit which is transmitted on the uplink after every slot in a potentially multi-slot downlink packet transmission. The bit indicates whether or not the mobile user has successfully decoded the transmitted packet Multi-slot transmissions are therefore terminated as soon as the mobile user successfully decodes a packet. As a result, H-ARQ scheme can increase the effective bit rate, improves the overall throughput of the system and implicitly reduce the excess $S I R$.

\subsection{Algorithm 4: Power Allocation Scheme in the HDR System}

In original HDR, when the base station chooses a mobile user, it always transmits signal with full power in order to improve the throughput of the system. But, when a mobile user is located close to a base station, the user acquires an excessive signal to interference power ration $(S I R)$ for a maximum achievable data rate. This excessive power results in lower $S I R$ for users at cell boundaries. By allocating lower power for user near a base station than the maximum power, other cell interference can be alleviated to users near cell boundaries [10] [11].

For a HDR system, base stations transmit data at a maximum power so that one can achieve a high data rate, which results in rate control instead of power control. The difference between the power control and the rate control has analogy with CDMA and TDMA. Generally, many users share the radio resources for graceful degradation in CDMA system. The best result for the same rate users is obtained under perfect power control. On the contrary, only one user can be served during a time interval and ideally 
has no same-cell interference but other-cell interference in TDMA systems. Thus, if one can minimize the other-cell interference, one can get better performance.

In HDR systems, an access terminal measures a pilot $S I R$ and sends a data rate command $(D R C)$ to an access network. Practically, the relationship between SIRs of pilot channel and achievable data rates can be calculated. Assume that the same level of maximum power is used for all $B S s . R_{\max }$ denotes the maximum achievable data rate in HDR systems and $\gamma_{m}$ is corresponding pilot $S I R$ for $R_{\text {max }}$. The conventional power allocation scheme for HDR systems work as follows. If the pilot SIR in a slot is greater than $\gamma_{m}, B S$ will support the maximum data rate at the slot. Because the requirement of pilot $S I R$ for maximum data rate is just $\gamma_{m}$, excessive $B S$ power for data is allocated to the access terminal under good channel condition. This excessive power also incurs other-cell interference, which results in lower SIR for access terminals in other cells to be unable to achieve the maximum data rate. An efficient power allocation scheme is proposed in [11]. $\gamma_{p}$ denotes the signal to interference ratio $(S I R)$ of pilot channel, $\gamma_{h}$ denotes the threshold of pilot $S I R$ with which $B S$ can support the maximum data rate when the level of $B S$ power for data is reduced to half of the power in the conventional scheme. If $\gamma_{p}$ is greater than $\gamma_{h}$, lower the $B S$ transmit power appropriate to meet the $S I R$ requirement for maximum data rate, other wise, transmit at maximum power at the $B S$.

The advantages of the proposed scheme are overall throughput increase, reduced average transmit power at $B S$, and outage reduction. However, in an implementation, a few bits are needed to indicate which data power level will be used.

In this scheme, the $B S$ allocates the radio signal power based on the $S I R$ of the pilot channel. But, it is difficult to determine the relationship between the $R_{\max }, \gamma_{p}$ and the transmission power. We will introduce a new scheme for power allocation based on the location of the users in the next chapter. 


\subsection{Algorithm 5: Inter-Cell Coordination Schemes}

Motivation of the inter-cell coordination schemes is to improve the "fairness" of the service seen by the users of an "HDR-like" system. In the downlink transmission of a CDMA system, system uses variable spreading gain to achieve different data rates for users based on their received SIRs. Although such schemes work efficiently, but they are not "fair" in that there is great inequality of the data rates achieved by various users. There are two schemes proposed in [12].

- Time Coordinated Transmission

In the scheme, system adopts round robin type of scheduling. Base stations in each neighboring cells share frame duration $T_{f}$, containing a coordinated period, $T_{c}$ and an uncoordinated period, $T_{u}$ as shown in Fig. 3.1.

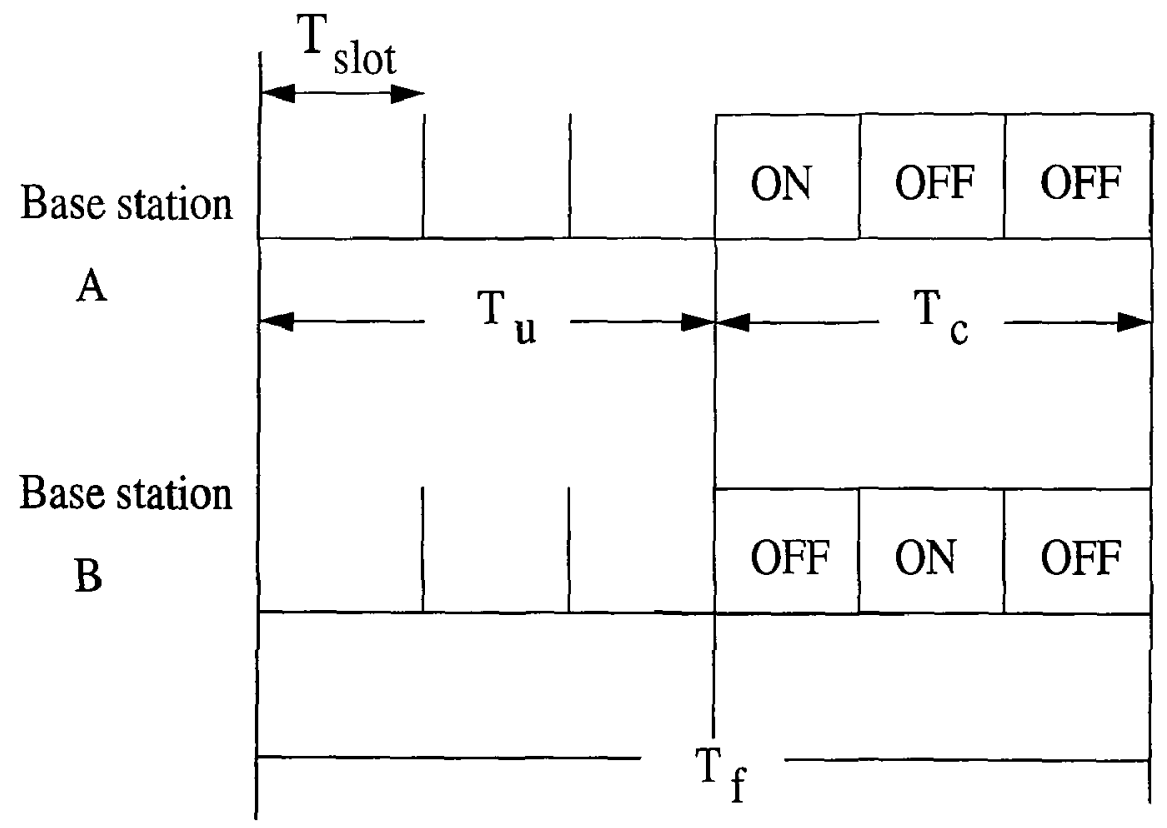

Figure 3.1: Frame Format in Time Coordinated Scheduling.

$$
T_{f}=T_{c}+T_{u}
$$

Each user is assigned a slot time during which only that user's signal is transmitted 
from a given base station. The number of user symbols per slot period depends on the particular radio propagation environment. During the coordinated period, $T_{c}$, each base station is "ON" for $N_{\text {on }}$ slots and "OFF" for $N_{\text {off }}$ slots in "round-robin" fashion. During coordinated period, only one of the bases in the neighborhood is active at any moment. The uncoordinated period contains a fixed number $\left(N_{u}\right)$ time slots, during this period, the base stations transmit signals to the users in their own cells simultaneously without considering the transmissions of the neighboring cells.

- Soft Hand-off Scheduling

Each base station has not only its own data which is transmitted to the users located in its own cell, but also it has a copy of the data of other base stations which should be transmitted to the users located in the neighboring cells. As previous time coordinated transmission scheme, each cell site has a period, $T_{u}$, during which the base station transmits only to its own users, and a period, $T_{c}$, during which it transmits the soft hand-off data to assist users belonging to other cells.

When coordination is used as the proposed scheme, the $S I R$ is improved during the coordinated period. On the other hand, this scheme works as "round-robin" fashion, some users may end up with extremely low throughput, despite receiving their fair share of the number of slots. However, because this scheme does not consider the other parameters, such as the channel conditions, the user distribution of the cell, it may degrade the performance of the system. Especially, the uncoordinated period $T_{u}$ is not suitable for reducing the interference. Otherwise, during the coordinated period $T_{c}$, each base "wastes" some of its time transmitting data to users in its own cell.

\subsection{Algorithm 6: A Coordination Downlink Schedul- ing in Linear Data-only Networks}

The quality-of-service requirements are also radically different between voice and data communications. Data traffic is usually less delay sensitive, but may have higher through- 
put requirements than voice during burst periods. These differences suggest that it may be beneficial to control the transmission rate for data users in a highly adaptive manner, rather than provide a constant guaranteed rate as for voice calls. In the coordination scheduling proposed in [14], the authors considered a linear data-only networks. The system models discussed in the scheme is as shown in Fig. 3.2, where, for base station $k$, we
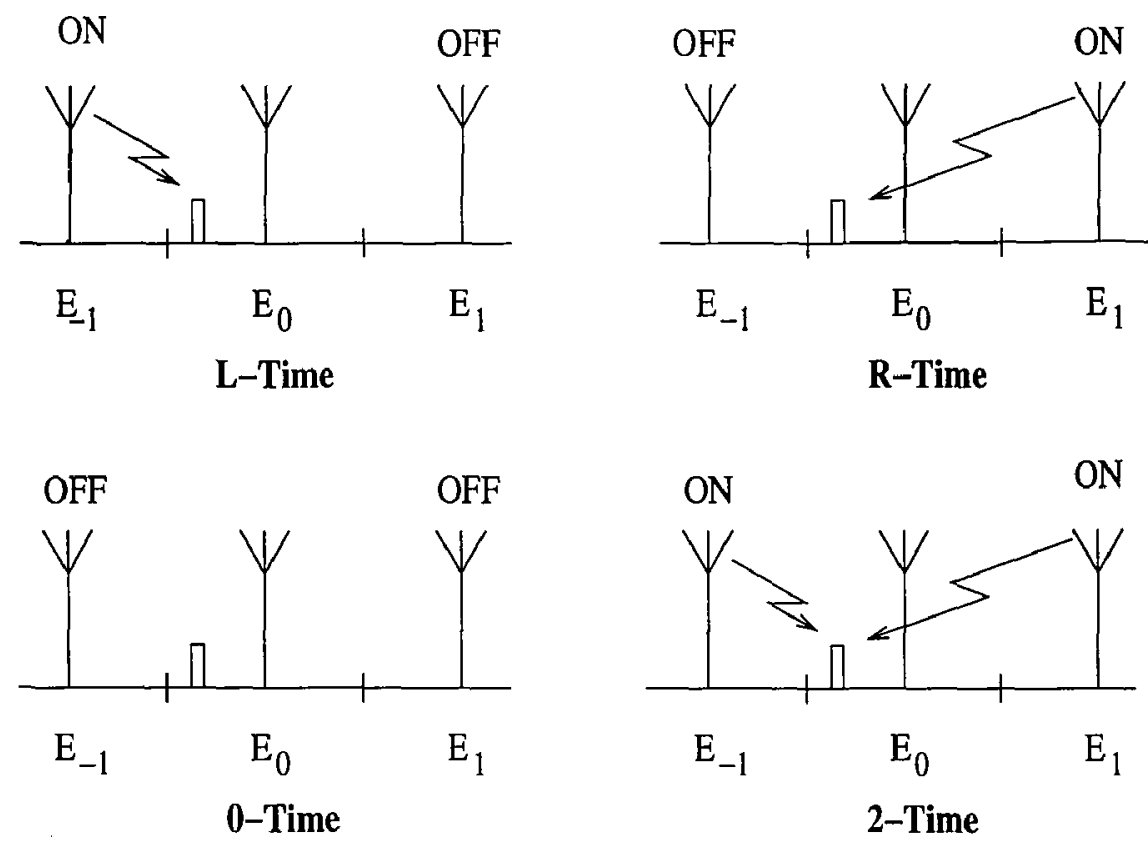

Figure 3.2: ON/OFF States of Cell $E_{0}$ 's Neighboring Base Stations during its L-time, R-time, 0 -time and 2-time.

refer to the time during which only its $k-1$ left neighbor is ON as its L-time. Similarly, the time during which only its $k+1$ right neighbor is transmitting, is called its R-time. The time during which both neighbors are $\mathrm{ON}$ is referred to as its 2-time. Finally, the time during which none of its neighbors is transmitting is termed as 0-time.

In the data-only networks, the base station, when ON, should transmit to only one data user at a time with maximum power for optimality. In this inter-cell scheduling, base station are allowed to alternate between ON-periods and OFF-periods in a a coordinated manner. There exist some optimality conditions to improve the throughput:

- Subsets of outer users on either side of the cell that should be served when only the neighboring base station in the opposite side is ON. 
- A subset of inner users in the center of the cell that should be served when both neighbors are ON.

- A subset of users in the intermediate regions should receive transmissions when both neighbors are OFF.

This scheme just considers the environment in the linear data-only networks. This model is relatively simple in comparison with a cellular system. On the other hand, when the base station is OFF, it wastes some of its time to transmitting the signals to users.

\subsection{Algorithm 7: Dynamic Channel-Sensitive Schedul- ing Algorithm}

The dynamic channel-sensitive scheduling algorithm takes advantage of the delay tolerance of data applications together with burst traffic characteristics [15]. The algorithms exploit the variations in the channel conditions and transmit to the user with the currently "best" channel in fading environment. The "best" user may be identified as the maximum-rate user when feasible rates are weighed with some appropriately determined coefficients. Interpreting the coefficients as shadow prices, or reward values, the optimal strategy may thus be viewed as a revenue-based policy, which always assigns the transmission slot to the user yielding the maximum revenue. It is a formidable task to calculate the optimal revenue vector directly, because it requires detailed information on the channel statistics. Instead, the adaptive algorithms for determining the optimal revenue vector online in an iterative fashion, without the need for explicit knowledge of the channel behavior.

The scheduling algorithm is able to maintain the target throughput ratios and can track sudden changes in the channel conditions or throughput targets well.

Depending on the specific situation, there are various performance criteria that might be adopted. One of the most common performance objectives is throughput maximization. This can be achieved by simply assigning each slot to the user with the currently 
highest feasible rate, such as the Best Channel First scheme (discussed in Algorithm 1). The disadvantage is that typically only a few strong users will ever be selected for transmission, causing starvation of all others. But, the Dynamic Channel-Sensitive Scheduling Algorithm focuses on maximum revenue. It introduces a class of revenue-based scheduling strategies. Suppose that there are rewards $w_{1}, \ldots, w_{M}$ per bit transmitted to the various users. A revenue-based strategy assigns the $n$th transmission slot to the user $m^{*}(n)$ with the current maximum rate-reward product, i.e.,

$$
m^{*}(n)=\max \left[w_{m} R_{m}(n)\right], m=1, \ldots, M
$$

where $M$ is the number of the data users served by a base station, $R_{m}(n)$ representing the feasible rate for user $m$ in the $n$th slot.

Hence, the above principle maximizes the revenue earned in each individual slot and the total cumulative revenue as well as the average revenue.

\subsection{Other Scheduling Algorithms}

There are also other HDR-like scheduling algorithms proposed for the high speed data transmission. For example:

- "Joint Power Control and Intra-cell Scheduling of DS-CDMA Non-real Time Data" [16]: The scheme uses time division so that users transmit in a one-by-one fashion within each cell. As well as, the scheme combines the intracell scheduling with a suggested distributed power control algorithm for the inter-cell interference management.

- "Opportunistic Transmission Scheduling With Resource-Sharing Constraints in Wireless Networks" [17]: the scheme exploits time-varying channel conditions and maximizes the system performance stochastically under a certain resource allocation constraint. 
- "Fair Scheduling in Wireless Packet Networks" [18]: Based on an adaptation of fluid fair queueing (FFQ) to handle location-dependent error bursts.

- "Downlink Resource Allocation and Pricing for Wireless Networks" [19]. The scheme applies to either a time-slotted system (e.g., HDR scheme) or a CDMA system whose feature is that the channel quality varies across the users. The algorithm uses a pricing scheme for the allocation of radio resources. In order to maximize revenue in such a system, the base station should allocate resources in a discriminatory manner, where different users are charged different prices based in part on their channel quality.

\subsection{Summary}

In this chapter, we discussed some of the current HDR and HDR-like scheduling schemes. Based on the characteristics of data traffic, i.e., delay tolerance, bursty traffic and high data rate requirement, base stations schedule the transmission to the mobile users in accordance with the scheduling algorithms. These algorithms exploit the time varying channel conditions and can be used to improve the performance of the wireless data networks under some certain constraint, such as throughput, priority, fairness and revenue of the CDMA systems.

Because of the characteristics of high speed data communication, designing a scheme to improve the throughput of the downlink transmission is very critical. For the downlink transmission, the major interference comes from the neighboring base stations. Especially, when users are located near the border of cells, they receive stronger interference power. However, there is no scheduling scheme which combines the coordination of transmissions and the power allocation for downlink transmission in the cellular communication system. In order to exploit the mobile channel condition and limit the neighboring cell interference, we proposed a new scheduling scheme- Coordinated Location-Based Downlink Scheduling Scheme (CLDSS) [13]. We will discuss the proposed scheme in detail in the next chapter. 


\section{Chapter 4}

\section{Coordinated Location-based Downlink Scheduling Scheme (CLDSS) with Partitioned Cells}

项 transmission rate requirements from base stations $(B S s)$ to mobile terminals (MTs) or vice versa. With the development of Internet, more and more mobile users access Internet via wireless networks. Most users require higher downlink (i.e, from BSs to $M T s$ ) rates than uplink (i.e, from $M T s$ to $B S s$ ) rates, which makes the nature of the data traffic to be asymmetric [6]. This thesis focuses on the downlink transmission and proposes a scheduling scheme which is called coordinated location-based downlink scheduling scheme (CLDSS) to support high data rate in cellular CDMA systems.

In CDMA systems as discussed in the previous chapter, all of MTs transmit in the same frequency band and access the transmission medium simultaneously. The original narrow-band signals are multiplied by a very large bandwidth signals - a set of unique spreading code sequences, that generates wide-band signals for transmission. Each $M T$ has its own pseudo-random (PN) codeword which is approximately orthogonal to all other codewords. In the downlink, each $B S$ transmits to all of its $M T s$ simultaneously and each user recovers the signal intended for it by despreading with its assigned code. Except for the desired signal that will be despreaded, all other $M T$ 's signals coming from its home base station as well as other base stations will remain spread and appear effectively as 
noise. This radio access technology for cellular communication makes the CDMA system to have many advantages, such as soft capacity limit and noise immunity. In 3G wireless networks, these advantages have been further exploited to support multimedia communication over the time-varying wireless channels. Since the capacity of CDMA systems is typically interference-limited, the transmit power must be carefully controlled. Otherwise, the signal-to-interference ratio (SIR) of on-going comnections will degrade which will result in higher bit error rates. In theory, it is possible, at least in the downlink, to use orthogonal signature sequences of codes for different $M T s$ to avoid interference among different codes. However, in practice, multi-path effects decrease the orthogonality at the receiver, and the signal intended for one user becomes interference for other users. The careful allocation of transmit power is one method of controlling the interference. On the other hand, the characteristics and requirements of high-rate data services and low-rate voice services are different; for example, two-way conversational speech requires strict adherence to symmetry. However, high-speed data that needs to be downloaded from a server can tolerate some delay, but it requires a very high throughput which makes the $B S$ to transmit signal to the intended user with large power. Based on the different requirements, it is desirable for a CDMA system to separate the low-rate services (such as voice) from the high-rate data services (such as file downloading) by means of scheduling $[6]$.

High data rate (HDR) scheduling scheme was proposed in $[6,7]$ mainly for non-real time data services. In a HDR system, $B S$ chooses one $M T$ at one time slot based on the channel conditions and the requirements of the user. The BS transmits data with maximum power in order to achieve higher data rate. However, there are some disadvantages. For example, the use of maximum power increases the interference to users in the neighboring cell (especially, the users located near the border). In the meantime, transmitting data to the users which are located closer to $B S$ with full transmission power can usually get much higher data rate than the maximum rate of the system. This thesis considers interference in HDR-like systems and proposes a scheduling scheme based on 
partitioning the cells and coordinating the transmission by the $B S s$ in different portions of the partitioned cells.

\subsection{System Model Description}

In order to take advantage of the channel conditions of the time-varying wireless environment and to deal with the problem of multi-user interference, scheduling is used in a time-slotted CDMA system. The concept of time-slotted CDMA system comes from the TDD (time-division duplex)-CDMA system, so called TD-CDMA system. In TDCDMA systems, both of the uplink and downlink transmissions use the same frequency channel. The $B S$ allocates certain time-slots for each transmission and does switching the transmission alternately between the two links. The TD-CDMA system provides the performance as well as the flexibility to fulfill the service requirements [20]. Fig. 4.1 shows the structure of the frame in the TD-CDMA system where the frame is divided by several time slots.

$10 \mathrm{~ms}$

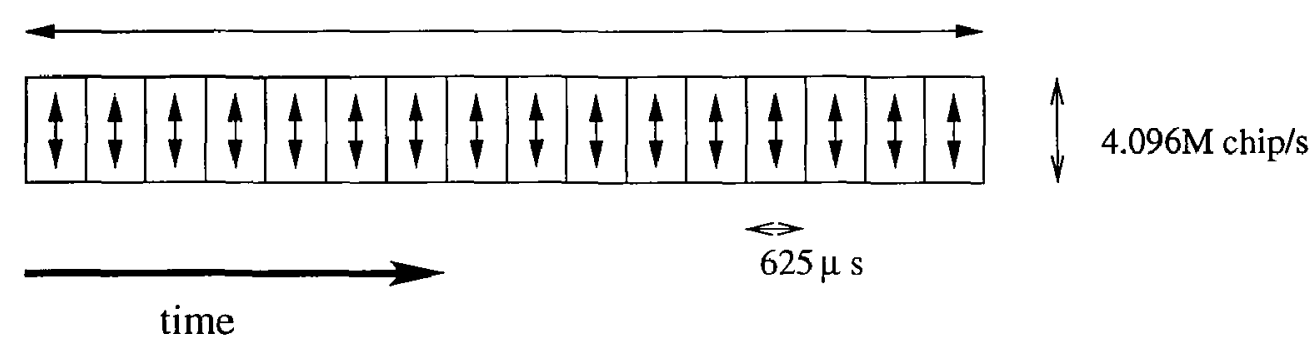

Figure 4.1: TD-CDMA Frame Structure.

In each slot, the $B S$ can schedule the transmission. For example, a $B S$ has the flexibility to choose the user, assign different data rate and decide the direction of transmission (uplink or downlink).

The proposed HDR-like scheduling scheme focuses on the downlink transmission; hence, all of the slots are allocated for downlink direction in the CDMA system in our work. This kind of TD-CDMA model is called time-slotted CDMA in the proposed scheduling scheme. The advantage of this model is that pooling of CDMA codes and 
TDMA time slots can easily provide the capacity demanded by a certain user (i.e., by the allocation of several codes and TDMA time slots, or both to a single user). The proposed scheduling scheme can adaptively allocate the time slots to different users based on the network load or data rate required by $M T s$ and also allocate the transmit power based on the user locations to control the interference.

We consider two neighboring cells in a square geometry (see Fig. 4.2). There are base stations $B S_{0}$ and $B S_{1}$ in the cell 0 and cell 1 respectively. We divide each cell into two co-centric areas as area $A$ (inner) and area $B$ (outer). The distances between the edges of outer and inner areas, and the center are $D$ and $d$ respectively as shown in Fig. 4.2.

The time axis is divided into slots and the transmission of data to users is coordinated by the $B S$ in each slot. The model can also be used in TD-CDMA or FD-CDMA, depending on the requirement for uplink and downlink transmission rate. The switching point in TD-CDMA can be adjusted or, if it is fixed as in FD-CDMA, time-slotted transmission helps implement the proposed scheduling scheme.

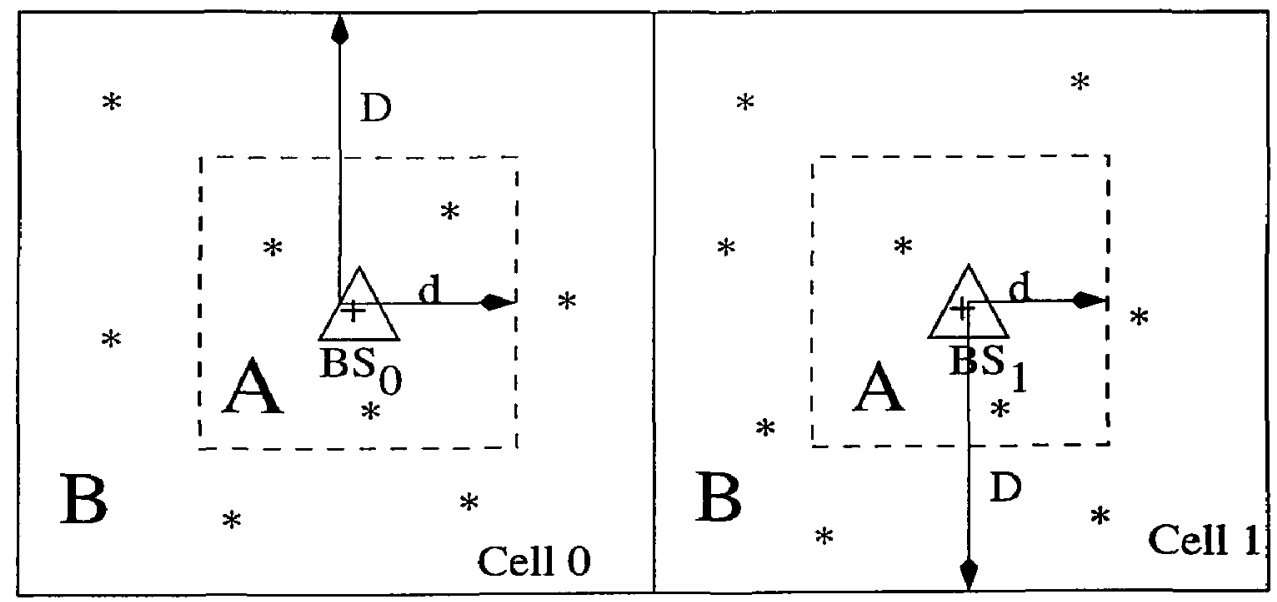

* --Mobile Terminal

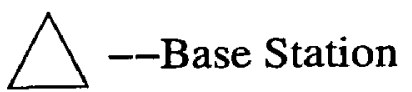

Figure 4.2: A Two-cell System Model.

We set that the $B S_{0}$ radiates power $P_{t 0}$ to $M T_{0}$ located in cell 0 and $B S_{1}$ radiates 
power $P_{t 1}$ to $M T_{1}$ located in cell 1 . We assume that the received power of each $M T$ bases on distance-dependent path loss and shadowing effect. The path gain between $B S$ and $M T$ is generally given as:

$$
g=\ell^{-\alpha} 10^{\frac{\xi}{10}}
$$

where $g$ is path gain, $\ell$ is the distance between $B S$ and $M T, \alpha$ is the propagation exponent, $\xi$ is the distortion parameter caused by shadowing between $B S$ and $M T$ which is a Gaussian random variable with standard deviation $\sigma$ and zero mean.

If we let the bandwidth of the system to be $W$ and the energy-per-bit to noise density ratio to be $E_{b} / N_{o}$, we get:

$$
\frac{E_{b}}{N_{o}}=\frac{P_{r e c} / R}{I / W}=\frac{W P_{r e c}}{R I}
$$

where $P_{\text {rec }}$ is the power received by the $M T$ from its home base station, $R$ is the transmission rate required by the user, $I$ is the total noise and interference power experienced by the user. $E_{b} / N_{o}$ is the value required for adequate performance of the modem and decoder. In order to decode a received signal with an acceptable probability of error, the $E_{b} / N_{o}$ should be more than or equal to a certain value. We use $\gamma$ to represent this threshold. So the maximum achievable rate is:

$$
R=\frac{W P_{r e c}}{\gamma\left(P_{I}+\eta\right)}
$$

where $P_{I}$ is the interference power experienced by the $M T$ under consideration $\eta$ is the noise power at the $M T$. Note that in a two-cell system model, $P_{I}$ is the received power from the neighboring $B S$.

\subsection{Intra Cell Scheduling: HDR-like Scheduling}

There can be several users in one cell. If a $B S$ transmits the signals to these users simultaneously, all of the users share the power of the $B S$ and interfere with each other 
[14].

In order to show the advantages of the intra cell scheduling scheme, we consider two cases as follows:

- Case A: Let us consider a time interval during which the total transmitted power levels are $P_{t 0}$ from $B S_{0}$ and $P_{t 1}$ from $B S_{1}$ respectively. We suppose that throughout the interval, a proportion $\phi_{i}$ of the total power $P_{t 0}$ (cell 0 ) is allocated to the $i^{\text {th }}$ data user, so that $\sum_{i} \phi_{i}=1$. From (4.3), the data rate received by user $i$ is:

$$
R_{i}^{A}=\frac{W}{\gamma}\left[\frac{\phi_{i} P_{t 0} g_{i}}{I_{i}+f_{i}\left(1-\phi_{i}\right) P_{t o} g_{i}+\eta}\right]
$$

where $f_{i}$ is orthogonality factor which represents the fraction of power transmitted by home $B S$ that appears as interference to user $i, f_{i} \in[0,1] . I_{i}$ is the interference from other base station to user $i, g_{i}$ is the path gain from home base station to user $i$.

- Case B: Here, each user $i$ is allocated the total power $P_{t 0}$ (cell 0 ), but only for a fraction $\phi_{i}$ of the interval. In other words, $\phi_{i}$ is used as a time-sharing rather than a power-sharing parameter. During the period that user $i$ receives signals from the $B S$, no power is used to transmit signals to other users in the cell, which means user $i$ does not receive interference from other user in the same cell at the same time. Hence, the rate received by user $i$ during that period is:

$$
R_{i}^{B}=\frac{W}{\gamma}\left[\frac{\phi_{i} P_{t 0} g_{i}}{I_{i}+\eta}\right]
$$

When comparing the two schemes, the throughput ratio is:

$$
\frac{R_{i}^{B}}{R_{i}^{A}}=1+\frac{\left(1-\phi_{i}\right) f_{i} P_{t 0} g_{i}}{I_{i}+\eta} \geq 1
$$

From the above inequality, the system should meet one of the below conditions at equality: 
- Only one user in the cell can be scheduled for transmission, so that $\phi_{i}=1$. In this condition, the case A and case B are same. Note that $0 \leq \phi_{i} \leq 1$.

- There are perfect orthogonality conditions between received signals and no multipath signals, so that $f_{i}=0$. However, in practice, because of the time delay and the dynamic fading, the multipath impacts the orthogonality and sometimes the influence is very high. In our analysis, we assume $f_{i}=1$, that is the worst case scenario for orthogonality between signals.

In conclusion, to improve the throughput of system, it is better to transmit to one user at a time rather than transmit to several users simultaneously. In other words, it is better to schedule one user in one cell (intra-cell scheduling) at one time as done in the proposed scheme.

\subsection{Inter Cell Scheduling: Proposed Scheduling Scheme (CLDSS)}

The purpose of the proposed scheduling scheme is to improve the downlink throughput of the CDMA system by reducing the interference from the neighboring cells. From the results in previous section, the proposed scheduling scheme chooses only one (data) user in each cell during one time slot. The proposed scheduling scheme will incur delays to many users and hence is appropriate for delay insensitive data users. Therefore, in the following we refer the user as data user.

Because the two-cells share the same frequency band and the multipath reduces the orthogonality of the pseudo-random codewords, there is co-channel interference between the two MTs located in different cells. The interference is based on the location of the user and the transmission power of the base station. When a $M T$ is located near the border of the cells, it would more likely experience interference from the other $B S$. When the distance between the user and the neighboring base station reduces, the interference power increases. Meanwhile, the distance between the user and its home base station 
increases, which makes the desired signal power to reduce. The result is the reduced SIR at the $M T^{\prime} s$ receiver. One of the methods to deal with this effect is to reduce the transmission power of the neighboring $B S$ at this time and to keep a certain level of transmission power of its home base station to the desired user.

On the other hand, when a $M T$ is located close to the $B S$, usually, the channel condition is better due to closer distance. Since the system has a preset maximum transmission rate (for example, $2 \mathrm{Mbps}$ ), it does not need full power to transmit data. Therefore, we can allocate part of the maximum power to the $M T$ located in the inner area. There are two advantages of this technique: it can reduce the interference to other $M T s$ located in the neighboring cell as well as it can save their battery power.

The proposed scheduling algorithm works as follows:

- The total maximum power available at both base stations is $P$.

- The system allocates the number of time slots to $M T_{s}$ located in the inner and outer area depending on the distribution of the users in the cells. For example, if there are $20 \%$ users located in the inner area, the system will allocate $20 \%$ time slots to the users which are located in the inner area, and other $80 \%$ time slots are assigned to the users located in the outer area. We assume that using the latest technologies in locating the mobile users, base station can estimate these numbers and hence determine the slot allocations accordingly.

- The two base stations in the cells system transmit data at the same time sharing the same frequency spectrum, but each $B S$ chooses only one $M T$ in its own cell in a time slot.

- When $B S_{0}$ transmits data to $M T_{0}$ which is located in the outer area (area $B$ ) of cell 0 with power $P, B S_{1}$ transmits data to $M T_{1}$ located in the inner area (area $A$ ) of cell 1 with power $\varphi \times P$, where $\varphi$ is a power adjustment factor and $0<\varphi<1$. Because we consider the power level based on the traffic load which relates to the 
user distribution and the area of the cell, we set $\varphi$ as a function of the ratio of inner area $A$ and outer area $B$. The area of inner and outer area are $4 \times d^{2}$ and $4 \times D^{2}$ respectively, so that $\varphi=(d / D)^{2}$.

- When $B S_{0}$ transmits data to $M T_{0}$ located in the inner area (area $A$ ) of cell $0, B S_{0}$ allocates power $\varphi \times P$. In the same time slot, $B S_{1}$ chooses $M T_{1}$ which is located in the outer area $(\operatorname{area} B)$ of cell 1 and transmits data with power $P$.

- During a time slot, the base station chooses one user in one of the areas (area $A$ or area $B$ ) based on some criteria, e.g., random- or channel condition- or prioritybased user selection algorithm.

The scheduling scheme and power allocation in the proposed scheme can be described using Fig. 4.3 for different time slots.

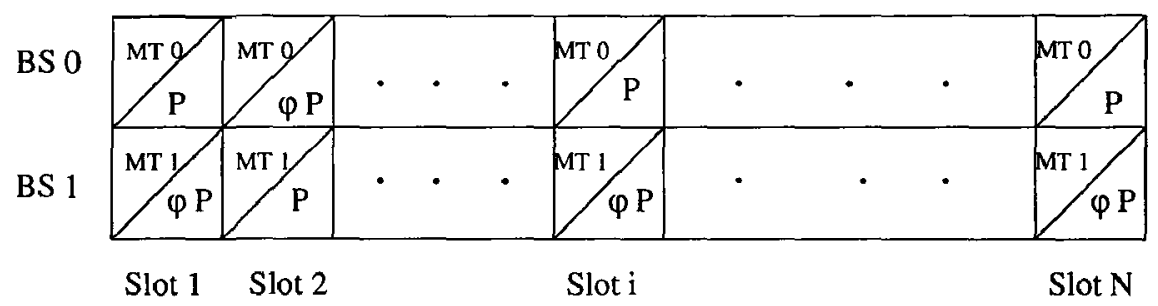

Figure 4.3: Scheduling and Power Allocation in CLDSS.

\subsection{Summary}

In order to improve the throughput of the high speed data communication service, we proposed a new scheduling scheme (CLDSS) based on the characteristics of wireless communication. In our proposed scheduling scheme, we employed time slots in CDMA systems; The transmission from a cell was coordinated with the transmission of the neighboring cell. We divided a cell as inner area (close to the base station) and as outer area (close to 
the border). We involved a power adjustment factor $(\varphi)$ to control the signal power allocated to the users in the inner or outer areas. In one time slot, the base station choosed one user from its own cell. We allocated time slots to the users depending on the user distribution in the cell. The proposed scheme can improve the perform of the CDMA system by reducing the interference from the neighboring cell. In the next chapter, the proposed CLDSS scheme is analyzed for throughput. 


\section{Chapter 5}

\section{Analysis of the CLDSS}

$\mathrm{B}$ ASED on our assumptions (see Section 4.1) and neglecting background noise, the received power and interference power for $M T_{0}$ are respectively in our system using the CLDSS scheme:

$$
\begin{gathered}
P_{r e c}=g_{00} P_{t 0}=P_{t 0}\left(\ell_{00}^{-\alpha} 10^{\frac{\xi_{00}}{10}}\right), \\
P_{I}=g_{01} P_{t 1}=P_{t 1}\left(\ell_{01}^{-\alpha} 10^{\frac{\xi_{01}}{10}}\right),
\end{gathered}
$$

where $g_{i j}$ is the path gain between $M T_{i}$ and $B S_{j}, \ell_{i j}$ is the distance between $M T_{i}$ and $B S_{j}, \xi_{i j}$ is the distortion parameter caused by shadowing between $M T_{i}$ and $B S_{j}$, which is a Gaussian random variable with standard deviation $\sigma$ and zero mean. Similarly, the received power and interference for $M T_{1}$ can be expressed.

\subsection{Analysis of Average Throughput of a System with the CLDSS}

Throughput is expressed as the number of bits passing through a data communication system per period of time. If the distance and shadowing are independent random variables, we can derive the mean rate (throughput) of a cell as follows from (4.3):

$$
E(R)=\left(\frac{W}{\gamma}\right) E\left[\frac{P_{t 0}}{P_{t 1}}\left(\frac{\ell_{00}}{\ell_{01}}\right)^{-\alpha}\right] E\left[10^{\frac{\xi_{00}-\xi_{01}}{10}}\right] .
$$


where $E$ is the expectation.

There have been many studies showing the capacity gain of CDMA, in which uniform distribution of the user population was assumed across the coverage area. Compared to the more general case of non-uniform user distribution, the use of uniform distribution model usually overestimates the capacity of the system.

In the proposed scheduling scheme, CLDSS assumes non-uniform distribution of users in each cell to analyze the capacity of the system.

The model of the non-uniform distribution of users can be described as follows:

- The total number of users in a cell is $N$.

- The cell is divided into two parts, as shown in Fig. 4.2.

- The distribution of users in area $A$ and area $B$ are uniform respectively with probability density $\rho_{a}$ and $\rho_{b}$ as shown in Fig. 5.1. Though this user distribution model does not capture the real nature of the practical system, it can be useful to understand the effect in the system capacity with non-uniform distribution of users.

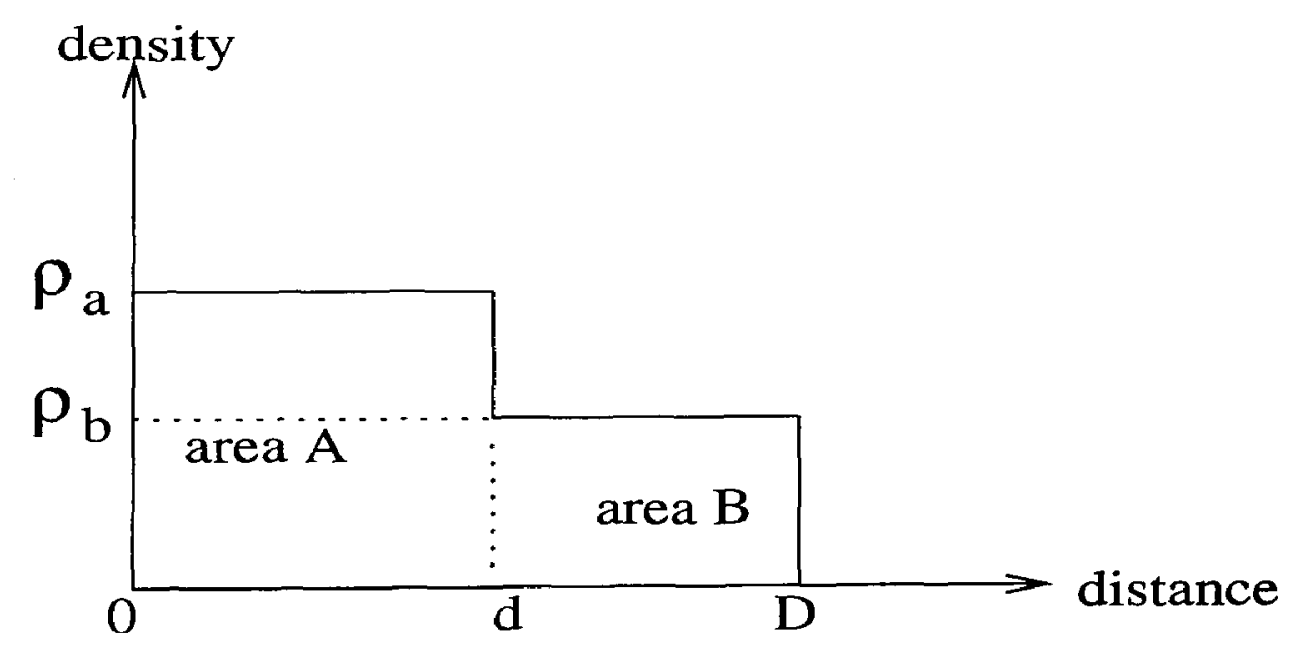

Figure 5.1: The Density Function of User Distribution in a Cell.

Since $W$ and $\gamma$ are assumed to be constant, for convenience, using the normalized throughput as $T$ (throughput $R$ is divided by $W / \gamma$ ), we analyze the average throughput of system. 
From (5.3), the normalized throughput is,

$$
T=\frac{1}{N}\left[\iint\left(\frac{\ell_{00}}{\ell_{01}}\right)^{-\alpha} \rho_{a} \varphi \times d A+\iint\left(\frac{\ell_{00}}{\ell_{01}}\right)^{-\alpha} \rho_{b} \frac{1}{\varphi} \times d B\right] E\left[10^{\frac{\xi_{00}-\xi_{01}}{10}}\right]
$$

where $\varphi$ is a power adjustment factor which is the ratio of the assigned power to a user located in the inner area (area A) and the assigned power to a user located in the outer area (area B). In the proposed scheduling scheme, let $\varphi$ depend on the ratio of the area of the inner part (area A) and the area of the outer part (area B) in one cell, so $\varphi=(d / D)^{2}$, hence, $0 \leq \varphi \leq 1$. Note that $\varphi=1$ corresponds to the ULISS scheme which will be discussed later.

Because $\xi_{00}$ and $\xi_{01}$ are assumed to be independent, letting $\xi_{00}-\xi_{01}=\chi$, the difference $\chi$ is a Gaussian random variable with zero mean and variance $2 \sigma^{2}$.

In general, in a CDMA system, when the achieved SIR is less than a certain value, the system can not effectively decode the signals. For this reason, CLDSS scheme sets up a condition for transmission, that is, when the SIR is less than a certain value, the $B S$ should stop to transmit any information to the user until the end of time slot. CLDSS scheme sets the threshold of SIR as $\tau$. The relationship between the parameters of the system is as follows:

$$
S I R=\frac{P_{r e c}}{P_{I}} \geq \tau .
$$

In accordance with the CLDSS scheme, the transmission power assigned by the base station is dependent on the location (that is affected by the distance and shadowing of the user), so that the expression of the condition to transmit is different between area $\mathrm{A}$ and area $B$.

- For the user located in the area A:

$$
\frac{P_{r e c}}{P_{I}}=\varphi \times\left(\frac{\ell_{01}}{\ell_{00}}\right)^{\alpha} \times 10^{\frac{\chi}{10}} \geq \tau
$$


From (5.6) we can get,

$$
\chi \leq 10 \times \alpha \log \left(\frac{\ell_{01}}{\ell_{00}}\right)-10 \log \left(\frac{\tau}{\varphi}\right) .
$$

The condition can also be expressed as follows:

$$
\Phi_{a}\left(\chi, \frac{\ell_{01}}{\ell_{00}}\right)= \begin{cases}1, & \text { if } \chi \leq 10 \alpha \log \left(\frac{\ell_{01}}{\ell_{00}}\right)-10 \log \left(\frac{\tau}{\varphi}\right) \\ 0, & \text { otherwise }\end{cases}
$$

where function $\Phi_{a}($.$) works as a switch of the successful transmission. In other words,$ during the period when $\Phi_{a}()=$.1 , base station transmits signals to a user, and the $S I R$ is high enough to get useful data, that has contributed to the throughput of the cell. Otherwise, the users are not able to decode the base station's signal with an acceptable probability of error, hence we do not count this part of data rate in the throughput.

Under this condition, the part (area A) of the mean of throughput of one cell can be derived from (5.6) as:

$$
T_{a}=\frac{1}{N}\left\{\iint\left(\frac{\ell_{00}}{\ell_{01}}\right)^{-\alpha} E\left[10^{\frac{\chi}{10}} \cdot \Phi_{a}\left(\chi, \frac{\ell_{01}}{\ell_{00}}\right)\right] \rho_{a} \varphi d A\right\} .
$$

For notational convenience, let $F_{a}\left(\chi, \ell_{01}, \ell_{00}\right)$ represent $E\left[10^{\frac{\chi}{10}}, \Phi_{a}\left(\chi, \frac{\ell_{01}}{\ell_{00}}\right)\right]$. Then,

$$
T_{a}=\frac{1}{N}\left[\iint\left(\frac{\ell_{00}}{\ell_{01}}\right)^{-\alpha} F_{a}\left(\chi, \ell_{01}, \ell_{00}\right) \rho_{a} \varphi d A\right]
$$

where

$$
F_{a}\left(\chi, \ell_{01}, \ell_{00}\right)=e^{(\sigma \ln 10 / 10)^{2}}\left\{1-Q\left[\frac{10 \alpha \log \left(\ell_{01} / \ell_{00}\right)-10 \log (\tau / \varphi)}{\sqrt{2} \sigma}-\frac{\sqrt{2} \sigma \ln 10}{10}\right]\right\}
$$

and 


$$
Q(x)=\frac{1}{\sqrt{2 \pi}} \int_{x}^{\infty} e^{-y^{2} / 2} d y
$$

The function $F_{a}\left(\chi, \ell_{01}, \ell_{00}\right)$ shows that it is a function of $\ell_{01}$ and $\ell_{00}$.

- Similarly, for user located in the area B of a cell:

The condition function of transmission is,

$$
\Phi_{b}\left(\chi, \frac{\ell_{01}}{\ell_{00}}\right)= \begin{cases}1, & \text { if } \chi \leq 10 \alpha \log \left(\frac{\ell_{01}}{\ell_{00}}\right)-10 \log (\tau \varphi) \\ 0, & \text { otherwise }\end{cases}
$$

The part (area B) of the mean of throughput of one cell can be derived from (5.13) as:

$$
T_{b}=\frac{1}{N}\left[\iint\left(\frac{\ell_{00}}{\ell_{01}}\right)^{-\alpha} F_{b}\left(\chi, \ell_{01}, \ell_{00}\right) \frac{\rho_{b}}{\varphi} d B\right]
$$

where

$$
F_{b}\left(\chi, \ell_{01}, \ell_{00}\right)=e^{(\sigma \ln 10 / 10)^{2}}\left\{1-Q\left[\frac{10 \alpha \log \left(\ell_{01} / \ell_{00}\right)-10 \log (\tau \varphi)}{\sqrt{2} \sigma}-\frac{\sqrt{2} \sigma \ln 10}{10}\right]\right\}
$$

Because there is no $Q$ function as built-in function in Maple, we use the error function $\operatorname{erf}(\boldsymbol{x})$ to calculate the integral. The relationship between $Q$ function and the error function is as follows:

$$
Q(z)=\frac{1}{2}\left[1-\operatorname{erf}\left(\frac{z}{\sqrt{2}}\right)\right]
$$

- The mean of throughput of one cell is the sum of the two parts as follow:

$$
T=T_{a}+T_{b}
$$

where $T_{a}$ and $T_{b}$ are derived in (5.9) and (5.14), respectively. 


\subsection{Performance Evaluation - Numerical Results}

Because the proposed scheme is based on the location of the users, how to partition the cell is very important. In order to analyze the proposed scheduling scheme, first of all, we have to determine the distance between the center of the cell and the border of the inner area. One of the reasons is that the power adjustment factor $\varphi$ is a function of the normalized distance $d^{*}$ of the inner area. We define the normalized distance of the inner area as $d^{*}=\left(\frac{d}{D}\right)$. The other reason is how to allocate the time slots to the users in the inner and outer areas also depends on the user distribution of the cell. In other words, it is relative to the ratio of the users located in the inner and outer areas. The relationship between the average throughput $(T)$ of a cell and the normalized distance $d^{*}$ can be evaluated numerically. For example, when $\alpha=4, \sigma=8 \mathrm{~dB}, \tau=5, \rho_{a}=0.00005$ (users $/ \mathrm{m}^{2}$ ), $N=100$ and $D=1000 \mathrm{~m}$, the throughput using the CLDSS scheme can be approximately curve-fitted as $T=1.6 \times 10^{6} \times e^{-16 d^{*}}$, as shown in Fig. 5.2. This graph was obtained by evaluating (5.17), (5.14) and (5.9) numerically.

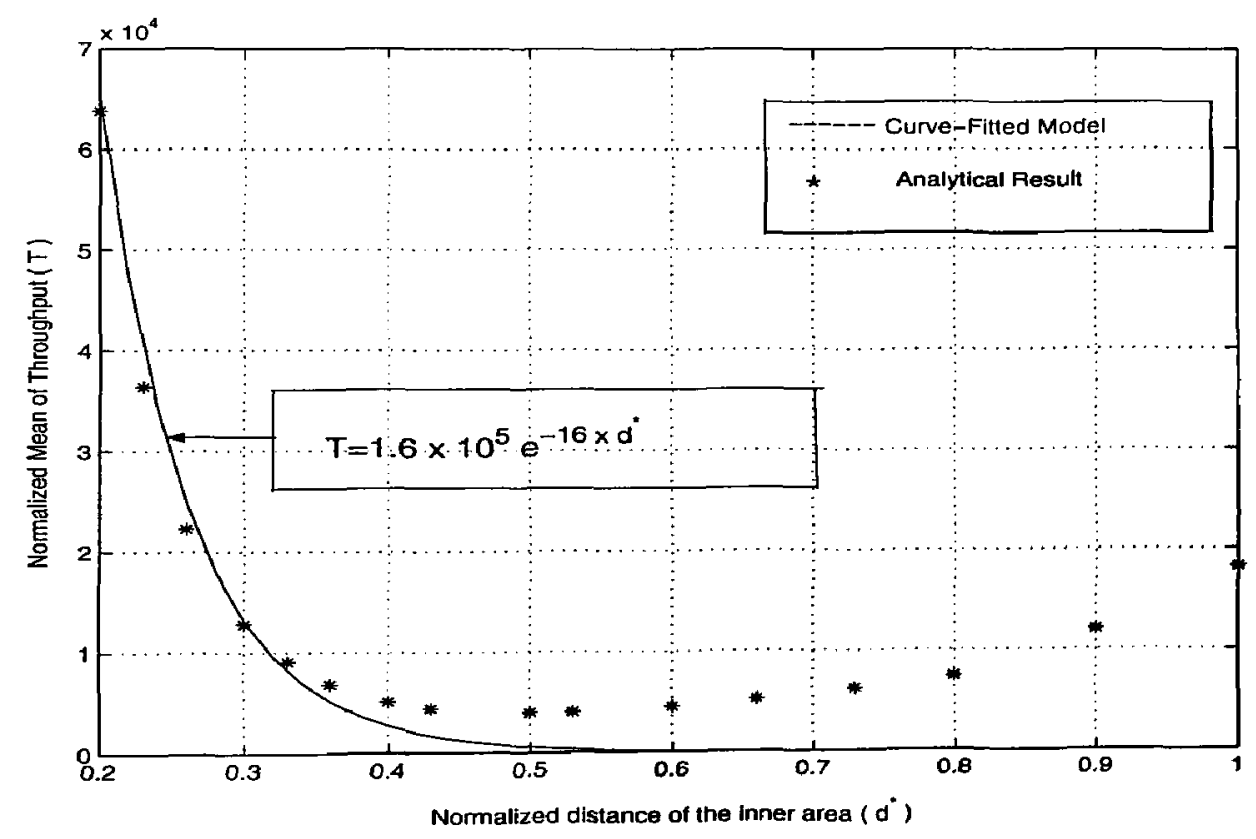

Figure 5.2: Curve-fitting of Average Throughput of CLDSS in a 2-cell System with Normalized Distance $d^{*}$.

The result represents the effect of the two parts: the average throughput is the sum 
of the contribution from the inner area and another from the outer area (which will be discussed later). There are some trends that can be observed from Fig. 5.2:

1. The normalized throughput increases as long as the normalized distance reduces. Hence, if one wants to get higher throughput, one should keep the normalized distance $d^{*}$ as small as possible.

2. In the limit when $d^{*}=1$, (or $d=D$ ), we turn the CLDSS scheme into the traditional uncoordinated location independent scheduling scheme (ULISS) where each base station schedules a user in its own cell without considering the location of the user.

3. When $d^{*} \leq 0.4$, the throughput of the CLDSS scheme can be analyzed using the curve-fitted formula $T=1.6 \times 10^{6} \times e^{-16 d^{*}}$ approximately.

The normalized throughput of the system is influenced by parameters, such as the density of the user distribution in the cell, the total users of the cell and environment of the wireless communication. When we optimistically set $d^{*}$, we can realize the influence on the throughput contributed by these parameters.

We compare the performance of the CLDSS scheme with two other scheduling schemes, which are Uncoordinated Location-Independent Scheduling Scheme (ULISS) and Coordinated Location-Independent Scheduling Scheme (CLISS).

- Uncoordinated Location-Independent Scheduling Scheme (ULISS):

In this scheme, we set $\varphi=1$. This means no power adjustment is done and each $B S$ transmits with full power $(P)$ to any $M T$ in any time slot without considering the location of the user. The transmissions in the two cells are independent, which means the two cells do not coordinate with each other. Additionally the base station randomly chooses the users in the inner or outer areas. To be consistent with the CLDSS scheme, the ULISS scheme allocates the time slots to the two areas (area A and area $B$ ) based on the user distribution of the two areas. For an instance, when $20 \%$ users are located in the inner area, the base station allocate $20 \%$ time slots 
to the users in the inner area. Within these allocated time slots, each base station selects a random user in either inner or outer area.

- Coordinated Location - Independent Scheduling Scheme (CLISS):

In this scheme, we set $\varphi=0.5$. The $B S$ transmits full power $(P)$ to the $M T s$ located in the outer area (area $B)$ and half power $(0.5 P)$ to the MTs located in the inner area (area A). In addition, while a base station transmits signal to the user located in the inner, the neighboring base station chooses the user located in the outer area to transmit signal (coordinated scheduling). Similarly, the percentage of the time slots allocated to the users in the inner (or outer) area depends on the percentage of the users located in the inner (or outer area). The main difference between CLISS and CLDSS is the power adjustment factor. In CLDSS, the power is allocated in proportion to the areas (or number of users) and in CLISS, equally in areas disregarding the number of users in these areas.

In summary, the parameters for the above three schemes can be seen in Fig. 5.3.

\begin{tabular}{|c|c|c|c|}
\hline & $\begin{array}{c}\text { ULISS } \\
\text { (traditional) }\end{array}$ & CLISS & $\begin{array}{c}\text { CLDSS } \\
\text { (proposed) }\end{array}$ \\
\hline $\begin{array}{c}\text { Power adjustment factor } \\
\varphi\end{array}$ & 1 & 0.5 & $(\mathrm{~d} / \mathrm{D})^{2}$ \\
\hline Power & $\mathrm{P}$ & $\begin{array}{c}\text { area A: 0.5P } \\
\text { area B: } \mathrm{P}\end{array}$ & area A: $\varphi \mathrm{P}$ \\
\hline Slots allocation & $\begin{array}{c}\text { based on user } \\
\text { distribution }\end{array}$ & $\begin{array}{c}\text { based on user } \\
\text { distribution }\end{array}$ & $\begin{array}{c}\text { based on user } \\
\text { distribution }\end{array}$ \\
\hline User selection & random & $\begin{array}{c}\text { area A: random } \\
\text { area B: random }\end{array}$ & area A: random \\
\hline Transmission B: random \\
\hline
\end{tabular}

Figure 5.3: Comparison of ULISS, CLISS and CLDSS Schemes. 
In the following numerical results, the total number $(N)$ of users in a cell is 100 . We take into account the far-field or Fraunhofer region [1] and hence do not count the area too close to the base station (the area is a square with side distance of $100 \mathrm{~m}$ ). Therefore, based on the shape of the cells shown in Fig. 4.2 and the user distribution model as in Fig. 5.1, the relationship between the parameters is:

$$
N=4\left[\rho_{a} \times\left(d^{2}-10000\right)+\rho_{b} \times\left(D^{2}-d^{2}\right)\right] .
$$

\subsubsection{Results with Uniform User Distribution}

In the case of uniform distribution of users, the density of the user distribution in the inner area $\left(\rho_{a}\right)$ is same as the density in the outer area $\left(\rho_{b}\right)$. For example, if set the $D$ is $1000 \mathrm{~m}$, then $\rho_{a}=\rho_{b}=2.525 \times 10^{-5}$ (users $/ \mathrm{m}^{2}$ ). We make the following observations:

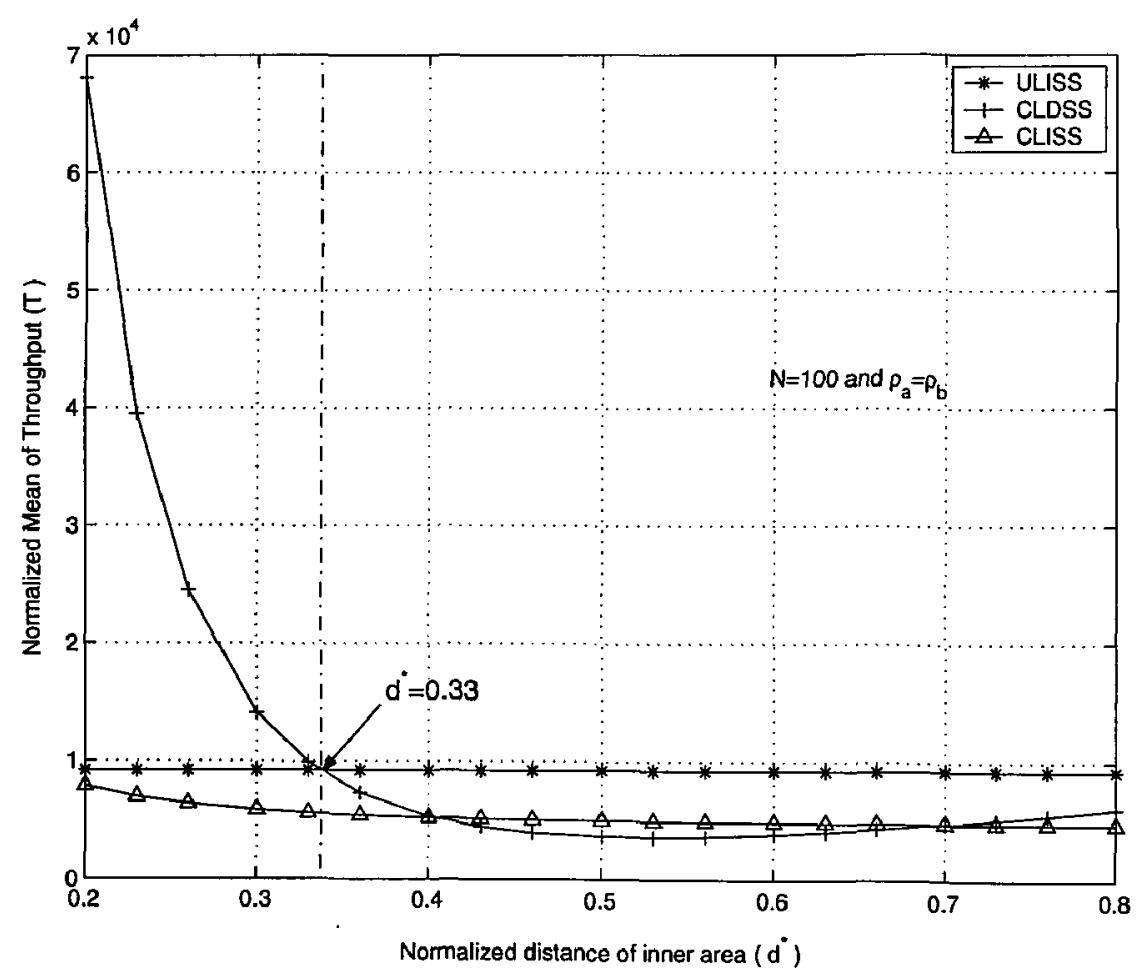

Figure 5.4: Throughput with Uniform User Distribution. 
- When the normalized distance $\left(d^{*}\right)$ of the inner area is less 46 performance of the proposed scheduling is superior. For example, if the $d^{*}$ is equal to 0.2 , the normalized mean of throughput using the CLDSS scheme is $6.81 \times 10^{4}$ and that using the ULISS scheme is $9.21 \times 10^{3}$, so the average throughput is improved $640 \%$ by the proposed scheduling scheme. However, the users should not be too close to the base station, in words, $d^{*}>>0$. Firstly, it is clear that (4.1) does not hold for $\ell=0$, and the system model evaluates the throughput for values of distance (between base station and user location) which are in the far-field of the transmitting antenna. Secondly, because we employ a power adjustment factor $\varphi$, if $d^{*}$ is very small, then the radio signal power assigned to the users located in the inner area would not be large enough to transmit data that can be decoded usefully in the presence of noise.

- If $d^{*}>0.33$, the performance of the ULISS scheme is better than the CLDSS scheme. The reason is that the average throughput of a cell is comprised of two parts: the throughput contribution from the inner area and outer areas. Comparing with ULISS scheme, because of the effect of factor $\varphi$, the radio signal power to the users located in the inner area is reduced by $\varphi P$ when we cmploy the CLDSS scheme. At the same time, the base station of neighboring cell transmits signal with full power $P$ which is same as the transmission power used in the ULISS scheme. Under this condition, the desired signal power is reduced and the interference power is not changed. When we compare the CLDSS scheme with the ULISS scheme, the $S I R$ in using the CLDSS scheme is reduced. Therefore, the throughput of the inner area reduces, which is a sacrifice. Oppositely, we use the full powcr $P$ to transmit signals to the users located in the outer area, which makes the desired signal power to be improved. In the meantime, the neighboring base station assigns part of the power as $\varphi P$ to transmit signal to the user located in its own cell, which makes the interference to be reduced. In comparing with the ULISS scheme which always radios full power $(P)$, the $S I R$ in using the CLDSS scheme for the user located in 
the outer area is improved. So that the throughput of the outer area increases, which is a compensation. Fig. 5.4 shows us beyond this point $\left(d^{*}=0.33\right)$, the reduction of throughput provided by users in the inner area can not be compensated by the improvement of throughput provided by users in the outer area.

- There is a key point for the normalized distance, which is $d^{*}=0.33$. Therefore, we conclude that the normalized distance $\left(d^{*}\right)$ should be less than 0.33 for the CLDSS scheme under the condition with uniform distribution of users in a cell in order to improve the throughput comparing with the ULISS scheme.

\subsubsection{Results for Non-Uniform User Distribution}

We now analyze the performance of non-uniform user distribution in the cell. We set the density of the user distribution in the inner area $\left(\rho_{a}\right)$ to be either $1.7 \times 10^{-5}$ or $5 \times 10^{-5}$ (users $/ m^{2}$ ). From Fig. 5.5, we can analyze the average throughput by employing the proposed scheduling scheme (CLDSS) with that by employing no power adjustable scheme (ULISS).

From Fig. 5.5, it can be seen that:

- In the ULISS system, as the density $\rho_{a}$ decreases, which means more users are located far away from the base station and near the border of the cell area (if we fix the normalized distance of the inner area $d^{*}$ ), the throughput of the system reduces distinctly. But the change of the average throughput using CLDSS scheme is small.

- The change of the normalized mean of throughput with different density of the user distribution in the inner area using the ULISS scheme is much larger, when we compared it with the CLDSS scheme. For example, if we set the density $\left(\rho_{a}\right)$ to be $5 \times 10^{-5}$ (users $/ \mathrm{m}^{2}$ ) and $1.7 \times 10^{-5}$ (users $/ \mathrm{m}^{2}$ ) respectively and the normalized distance $\left(d^{*}\right)$ to be 0.33 , the change in throughput by using the ULISS scheme is $1.13 \times 10^{4}$, where the throughput with $\rho_{a}=5 \times 10^{-5}$ (users $/ \mathrm{m}^{2}$ ) is $1.77 \times 10^{4}$ and the throughput with $\rho_{b}=1.7 \times 10^{-5}$ (users $/ m^{2}$ ) is $6.37 \times 10^{3}$. But, the change 


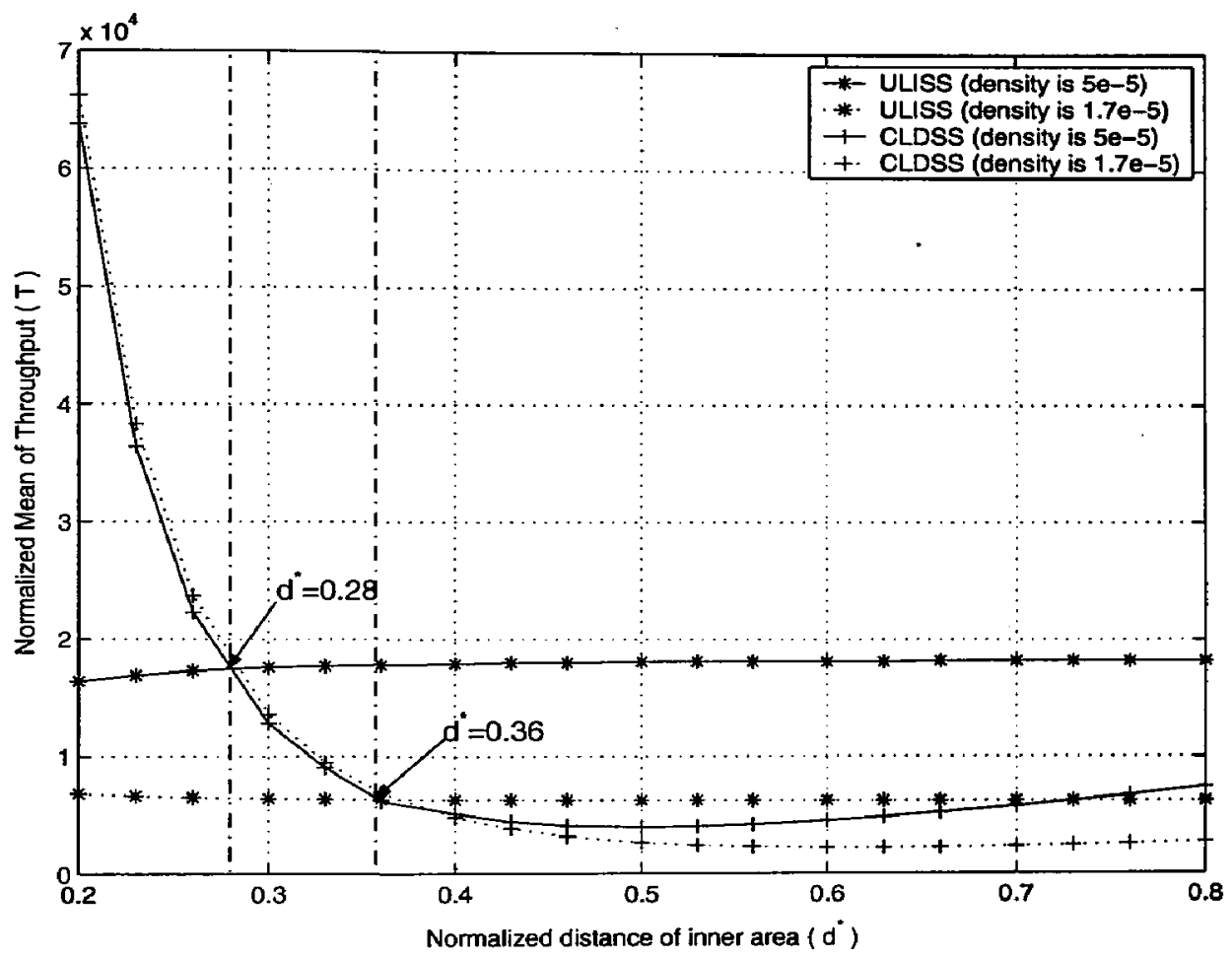

Figure 5.5: Throughput with the Non-uniform Distribution of Users. 
in throughput by using the CLDSS scheme is just 440 , where the throughput with $\rho_{a}=5 \times 10^{-5}$ (users $/ \mathrm{m}^{2}$ ) is $9.05 \times 10^{3}$ and the throughput with $\rho_{b}=1.7 \times 10^{-5}$ (users $/ m^{2}$ ) is $9.49 \times 10^{3}$.

- The effect of user density on the throughput using the proposed scheduling scheme is very small., When the normalized distance $d^{*}$ is less than 0.4 , the difference between the two throughput with the different density is minimal. Hence, the proposed scheduling scheme can improve the fairness of the system in terms of providing throughput.

- Similarly, because of the effect of the power adjustment factor $\varphi$ discussed before, there is also a key point $\left(d^{*}=0.28\right)$ for the non-uniform user distribution system. When $d^{*}<0.28$, the throughput using the CLDSS scheme is much better than that of the ULISS system. For example, when $d^{*}=0.2$ and $\rho_{a}=1.7 \times 10^{-5}$ (users $/ \mathrm{m}^{2}$ ), the throughput using the CLDSS scheme is $6.62 \times 10^{4}$ and that of the ULISS scheme is $6.83 \times 10^{3}$. But, beyond this point, when more users are located near the base station, the throughput of the ULISS scheme is better, e.g., when $d^{*}=0.3$ and $\rho_{a}=5 \times 10^{-5}$ (users $/ m^{2}$ ), the throughput of the ULISS scheme is $1.76 \times 10^{4}$ and that of the CLDSS scheme is $1.28 \times 10^{4}$.

Based on the results discussed before, there are some key points of the normalized distance of the inner area. That is, when we set the normalized distance $d^{*}$ to be less than these key points, the throughput of the system using the CLDSS scheme is better than the ULISS scheme. The key points are affected by the user density of the inner area. The relationship between the key points and the user density is shown in Fig. 5.6.

\subsubsection{Effect of User Density}

We let the normalized distance of inner area $\left(d^{*}\right)$ to be 0.2 to analyze the influence of the density of the user distribution on the average throughput of the system. From Fig. 5.7, we can make the following observations: 


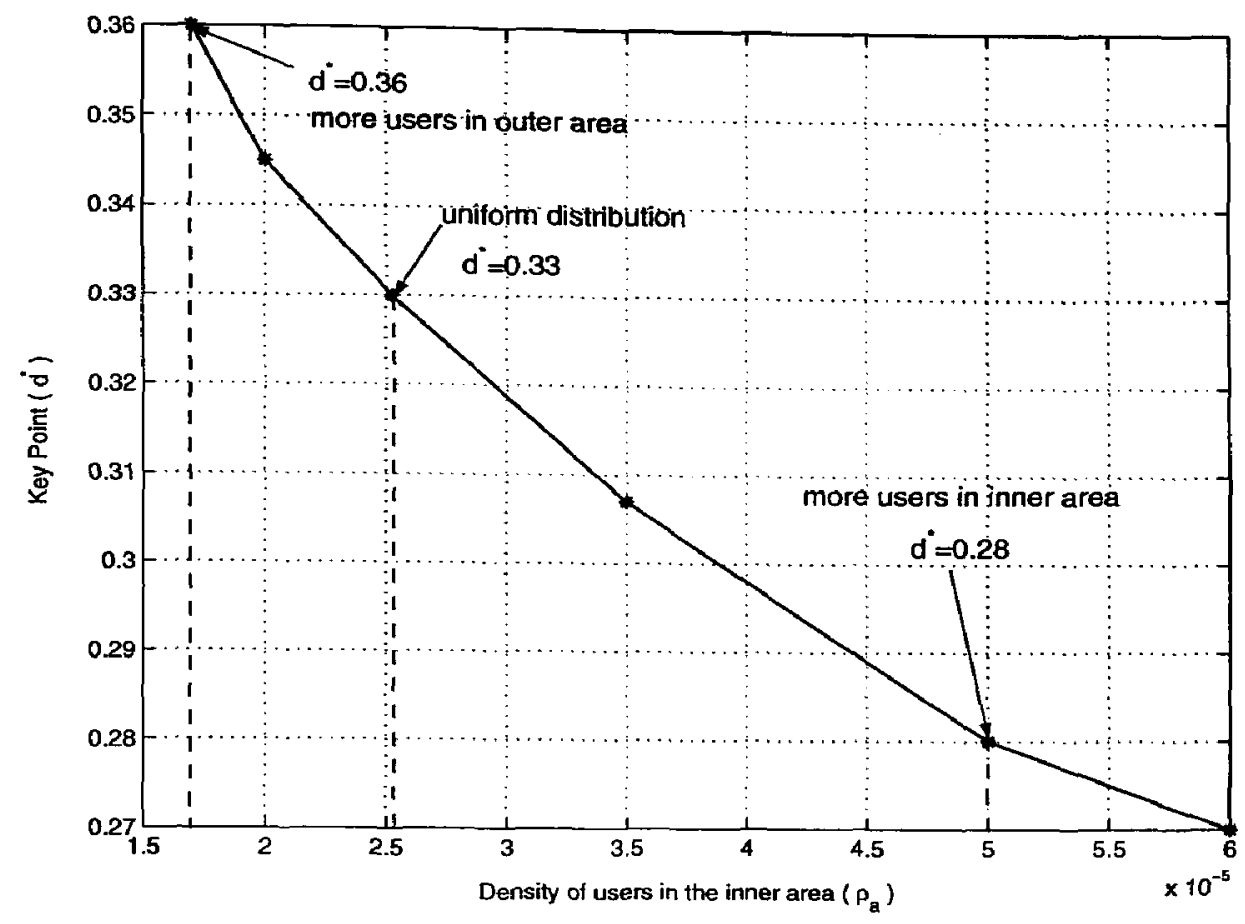

Figure 5.6: Relationship between Key Point and the User Density of the Inner Area

- The average throughput of the proposed scheduling scheme (CLDSS) is much better than that of the scheme (ULISS) when $\rho_{a}$ is less than $1.8 \times 10^{-4}$ (users $/ \mathrm{m}^{2}$ ). However, when $\rho_{a}$ is above this point, the average throughput of the ULISS scheme is better. One of the reasons is the effect of the power adjustment factor $(\varphi)$ and the other reason is that we allocate the time slots based on the user distribution in the two cells system. Therefore, when $\rho_{a}>1.8 \times 10^{-4}$ (users $/ m^{2}$ ), the throughput improved by the users located in the outer area can not be compensated for the throughput decreased by the users in the inner area. This is also shown in Fig. 5.8, which shows how the percentage of the users located in the inner arca affects the average throughput of the system. Similarly, when less than $21 \%$ users are located in the inner area with the normalized distance $d^{*}=0.2$, which means the area of the inner area is $3 \%$ of the the entire cell based on Fig. 4.2 and (5.18), the throughput of the CLDSS scheme is higher than that of the ULISS scheme. But, as more users are 


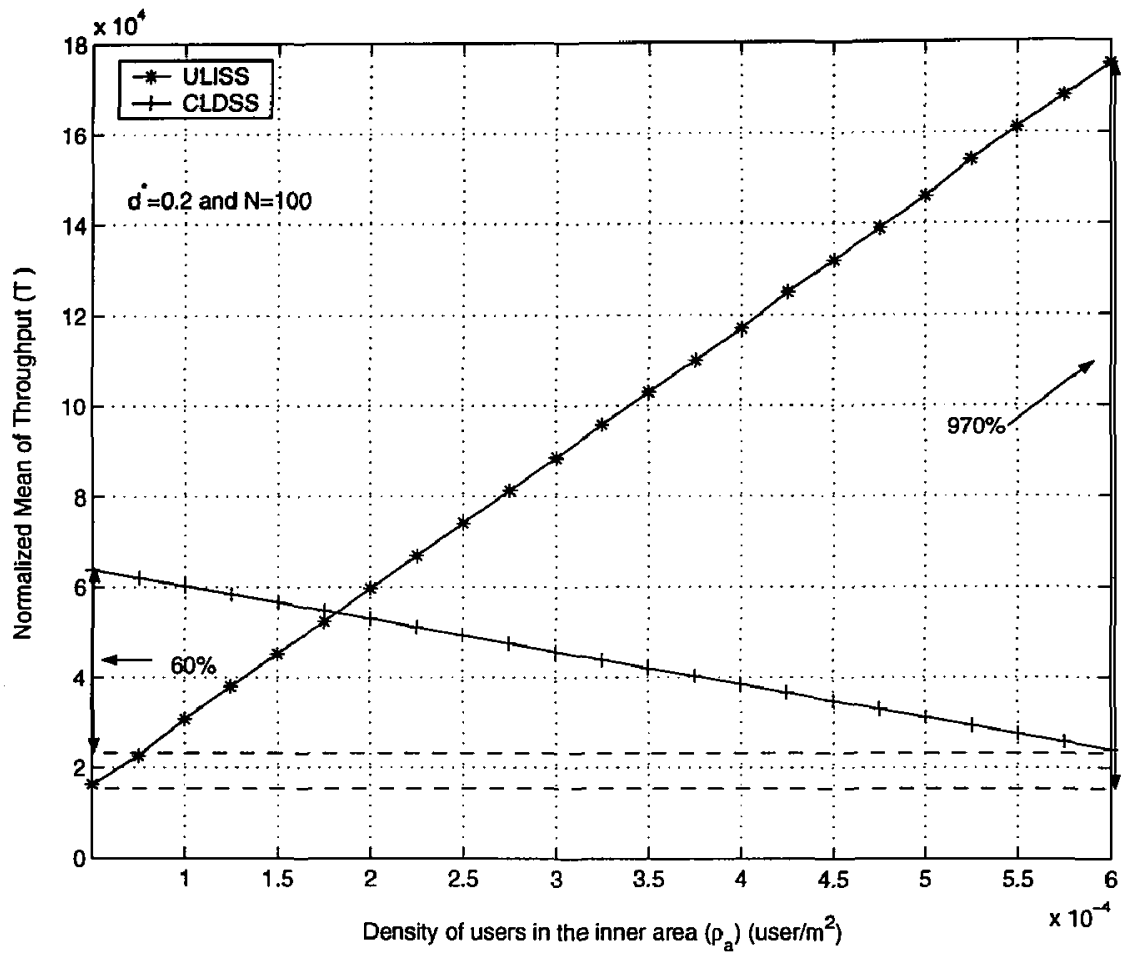

Figure 5.7: Throughput Affected by User Density. 
located in the inner area (when the percentage of users in the inner area is more than $21 \%$ ), the throughput of the ULISS scheme is better.

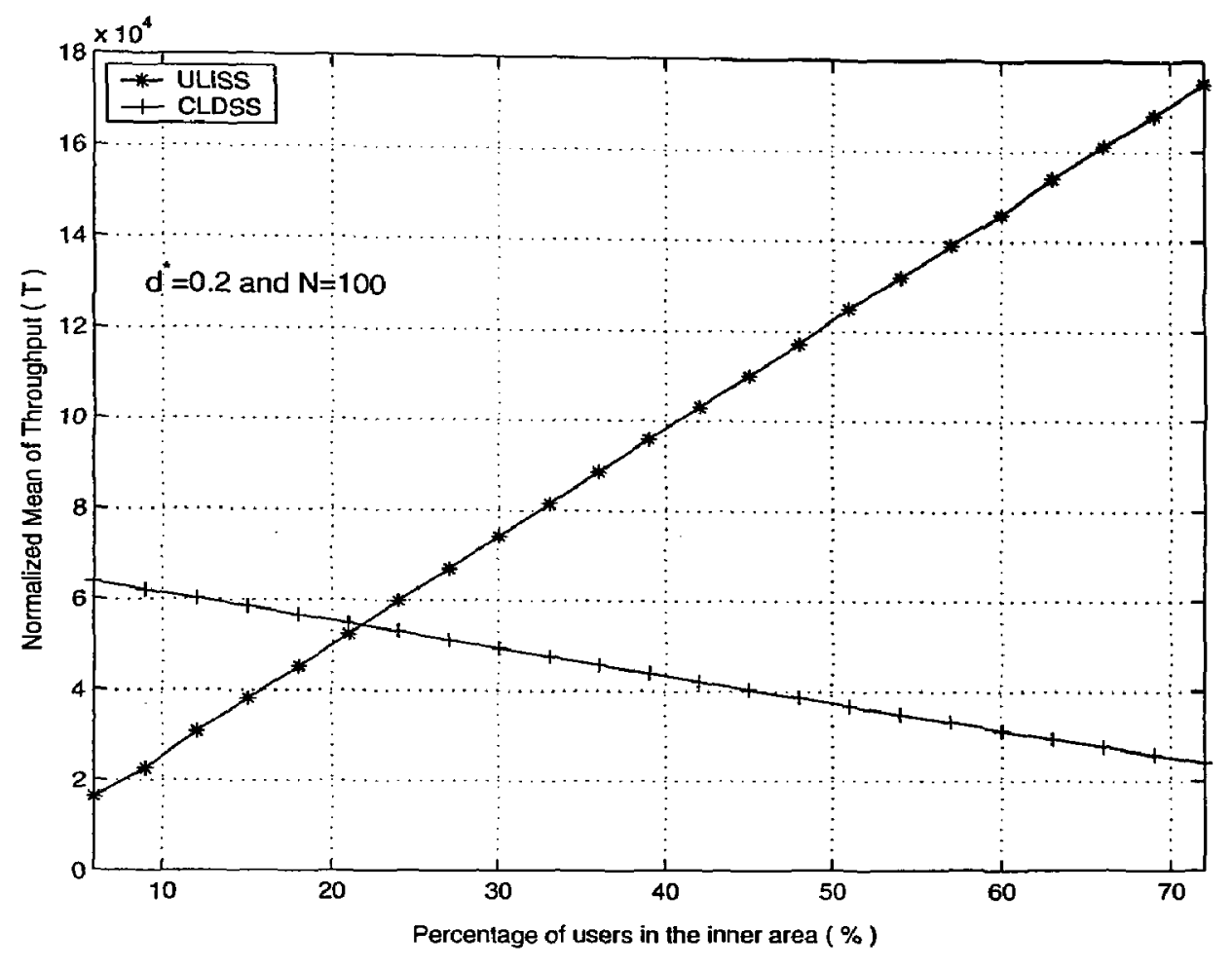

Figure 5.8: Throughput Affected by User Percentage.

- There is also a key point for the density of the user distribution in the inner area, it is $\rho_{a}=1.8 \times 10^{-4}$ (users $/ m^{2}$ ) corresponding to $d^{*}=0.2$.

- When $\rho_{a}$ reduces, the more users are located in the outer area (which is $97 \%$ of the entire cell) and the average throughput of the CLDSS scheme increases. As we assumed the normalized distance $\left(d^{*}\right)$ is 0.2 , the power adjustment factor $(\varphi)$ should be 0.04. In accordance with the CLDSS scheme, the base station assigns the full signal power $(P)$ to the users located in the outer area and $0.04 P$ to the users located in the inner area. As we discussed before, by comparing with the ULISS scheme, the power adjustment factor can improve the throughput of the outer area, but it also sacrifices some of the throughput of the inner area. When more users are 
located far away from the base station (i.e., the percentage of the users located in the outer area increases), the time slots allocated to the outer area are increased. Hence, the contribution of the throughput coming from the outer area increases. Contrary, the average distance between the base station and the users increase which make the throughput of the ULISS scheme to be reduced.

- The effect of user density on the CLDSS scheme is not significant comparing with the ULISS scheme. For example, using the CLDSS scheme, the throughput is $6.38 \times 10^{4}$ when $\rho_{a}=5 \times 10^{-5}$ (users $/ m^{2}$ ) and the throughput is $2.4 \times 10^{4}$ when $\rho_{a}=6 \times 10^{-4}$ (users $/ m^{2}$ ). The difference of throughput between the largest and smallest user density is $60 \%$. On the contrary, using the ULISS scheme, the throughput is $1.63 \times$ $10^{4}$ when $\rho_{a}=5 \times 10^{-5}$ (users $/ \mathrm{m}^{2}$ ) and the throughput is $1.75 \times 10^{5}$. The difference is $970 \%$ as shown in Fig. 5.7. Because of the characteristic of the CLDSS scheme, the CLDSS scheme can improve the fairness for the non-uniform distribution of users in the CDMA system. Typically, when more users are located near the border of the cell comparing with the ULISS scheme, and the CLDSS scheme can improve the average throughput of the system.

\subsubsection{Modification of the Proposed Scheme}

Because of the existence of the "key point", we combine the CLDSS scheme and ULISS scheme in order to achieve high throughput of the system.

- When the normalized distance of the inner area $\left(d^{*}\right)$ is less than 0.26 and the density of the user distribution in the inner area $\left(\rho_{a}\right)$ is less than $1.7 \times 10^{-4}$ (users $/ \mathrm{m}^{2}$ ) (or the percentage of users in the inner area is less than $21 \%$ ), we employ the CLDSS scheme; when $d^{*}$ and or $\rho_{a}$ are set beyond the "key point", we employ the ULISS scheme. The result of the optimization is shown in Fig. 5.9.

- In order to improve the fairness of the system with non-uniform user distribution in the cell, we involve fairness adjustment parameter $K$ into the power adjustment 


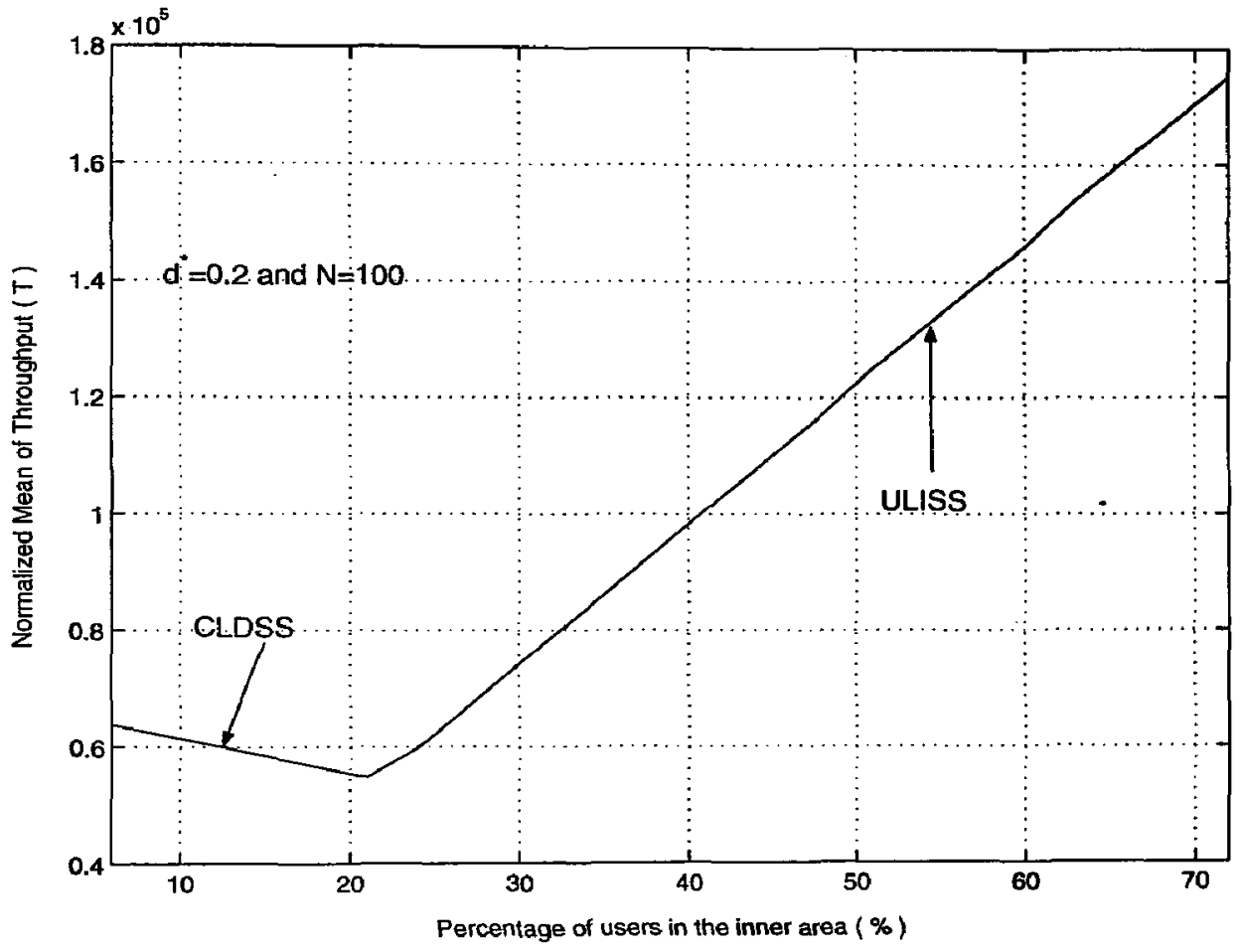

Figure 5.9: Throughput of Modified CLDSS. 
factor $\varphi$, as $\varphi=K \times\left(d^{*}\right)^{2}$. We can adjust the fairness of the system by changing the value of parameter $K$ (as shown in Fig. 5.10) for $d^{*}=0.2$ or $\left(d^{*}\right)^{2}=\frac{1}{25}$ :

Case 1: When we set $K=25$, the system employs the ULISS scheme. The fairness of the system is the worst.

Case 2: When we set $K=1$, the system employs the CLDSS scheme. The fairness of the system is better.

Case 3: When we set $K=2.75$, the average throughput of the system is minimally influenced by the distribution of users in the CDMA system. So that the fairness of the system is the best, but the average throughput is relatively low.

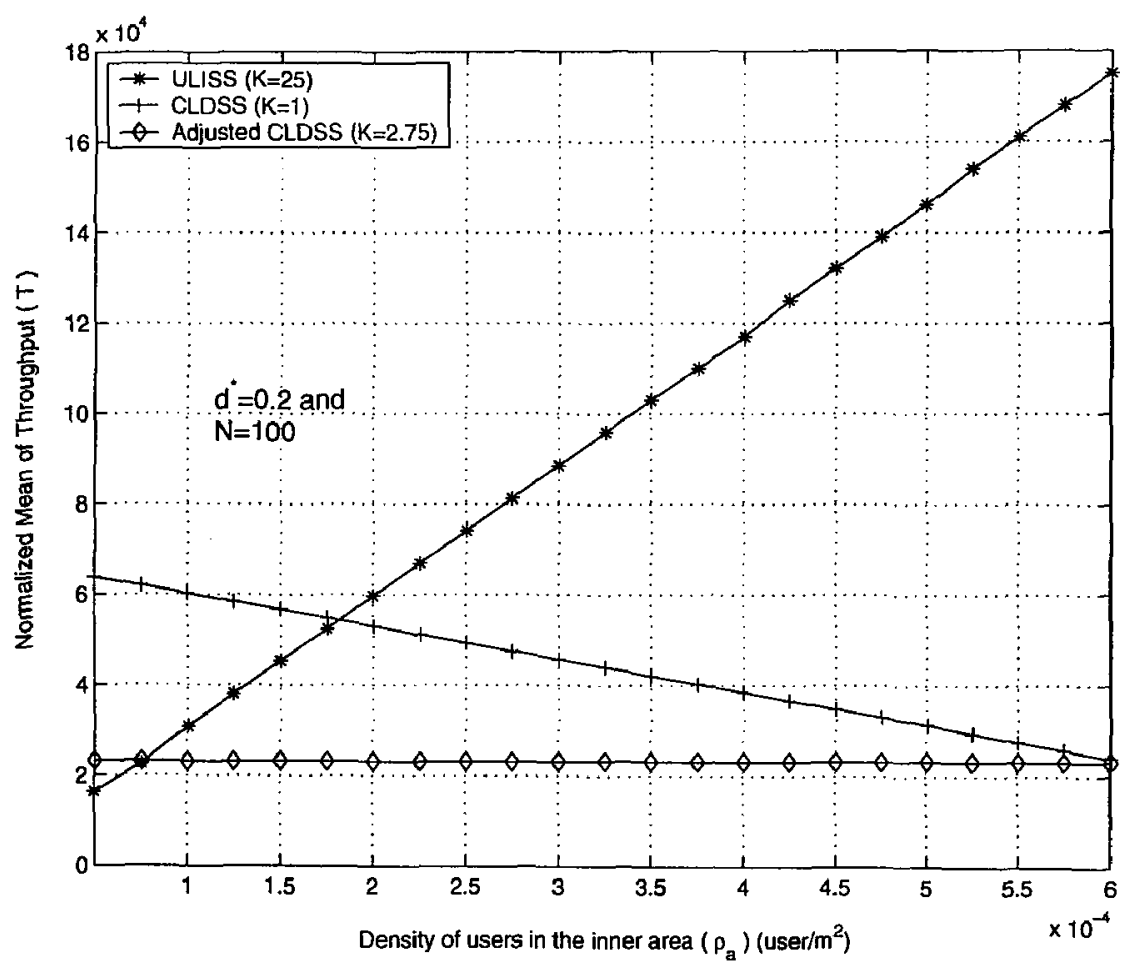

Figure 5.10: Throughput Impacted by Fairness Adjustment Parameter $(K)$. 


\subsubsection{Effect of the Number of Users in the System}

We let the normalized distance of inner area $\left(d^{*}\right)$ to be 0.2 , and vary the number of users in the cell. As the number of users increases, the results are shown in Fig. 5.11 and we can note the following:

- With the same number of users, using the CLDSS scheme, when the user density changes, the average throughput does not change very much.

- With the same number of users, using the ULISS scheme, when the user density changes, the average throughput changes significantly. When the density $\rho_{a}$ increases, more users are located near the base station and the channel conditions improve; as a result, the average throughput of system increases and vice verse.

- When we use the CLDSS scheme, the average throughput does not change very much, especially more users are located in the outer area. For example, when the number of users is 40 , if we employ the CLDSS scheme, the throughput is $5.84 \times 10^{4}$ with $\rho_{a}=5 \times 10^{-5}$ (users $/ \mathrm{m}^{2}$ ) and the throughput is $6.44 \times 10^{4}$ (users $/ \mathrm{m}^{2}$ ) with $\rho_{a}=1.7 \times 10^{-5}$ (users $/ \mathrm{m}^{2}$ ); If we employ the ULISS scheme, the throughput is $3.81 \times 10^{4}$ with $\rho_{a}=5 \times 10^{-5}$ (users $/ \mathrm{m}^{2}$ ) and the throughput is $1.42 \times 10^{4}$ with $\rho_{a}=1.7 \times 10^{-5}$ (users $/ m^{2}$ ). We can find that the difference of the throughput using the ULISS scheme is 5.5 times the difference of the throughput using the CLDSS scheme.

- When the number of users increases, the throughput of the CLDSS scheme increases. Oppositely, the throughput of the ULISS scheme reduces significantly. If the number of the users is more than 36 (see Fig. 5.11), the throughput of the CLDSS scheme is higher than that of the ULISS scheme.

- In the ULISS system, when the number of users changes, the average throughput of system changes more significantly than that of CLDSS, especially, when $\rho_{a}$ is high. For example, by using the ULISS scheme, when $\rho_{a}=5 \times 10^{-5}$ (user $/ m^{2}$ ), 
if the number of the users is 20 , the throughput is $7.42 \times 10^{4}$. If the number of the users is 200 , the throughput is $9.14 \times 10^{3}$, the difference of the throughput is $6.5 \times 10^{4}$. However, by using the CLDSS scheme, if the number of the users is 20 , the throughput is $4.9 \times 10^{4}$. If the number of the users is 200 , the throughput is $6.56 \times 10^{4}$. The difference of the throughput is $1.63 \times 10^{4}$. The reason is that the throughput of the ULISS system mainly bases on the channel condition and it can not be compensated.

- Because the throughput of the CLDSS scheme is almost independent of the change of the number of the users in the system, which makes the CDMA system to have "soft throughput".

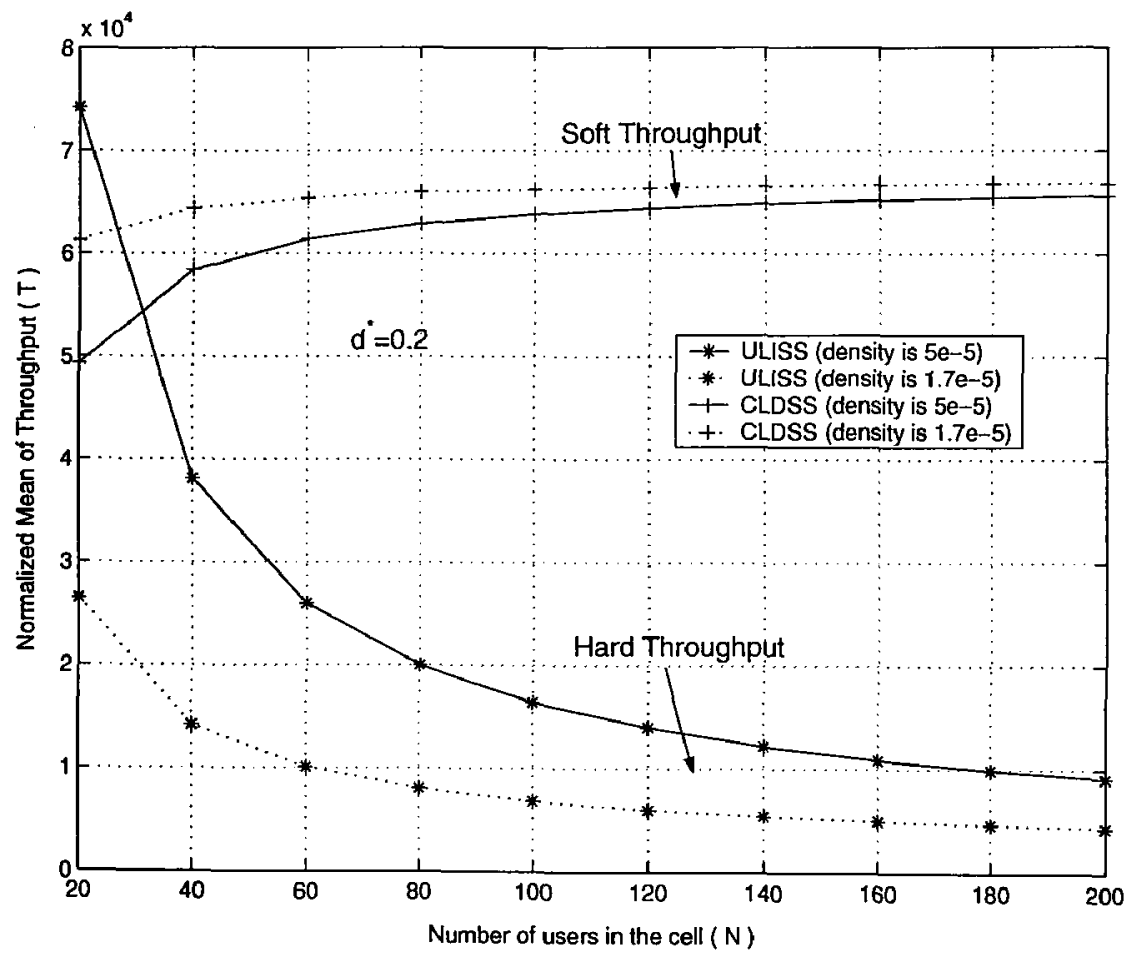

Figure 5.11: Throughput Affected by the Number of Users $(N)$. 


\subsection{Summary}

In accordance with the CLDSS scheme proposed in previous chapter, we coordinated the transmissions between the neighboring cells. Since we employed a power adjustment factor to control the radio signal power to users based on the locations and allocated the certain time slots to users based on the load of users, the CLDSS was useful to limit the neighboring cell interference and to improved the performance of the non-uniform user distribution system. In this chapter, we presented some numerical results from the analysis of the CLDSS. The proposed scheduling scheme has some advantages which were discussed using the numerical results. They are, it can improve the average throughput of the system, the fairness in the non-uniform user distribution and it has the feature of "soft throughput", especially, when the users ware located near the border of the cell. In the next chapter, we evaluate the performance of the proposed CLDSS scheme by simulation studies. 


\section{Chapter 6}

\section{Simulation and Results}

YYSTEM simulation is implemented to evaluate the performance of the proposed
CLDSS scheduling scheme. As discussed in the previous chapter, the CLDSS scheme coordinates the transmission between the inter-cell mobile terminals (MTs) in a downlink CDMA system. In the CLDSS scheme, a cell is divided into two flexible co-centric areas based on the traffic load in the cell. The base stations $(B S s)$ allocate the transmission power based on the distance between $M T s$ and $B S s$ with a power adjustment factor. When one $B S$ transmits data to a $M T$ which is located in the inner area with lower power, the neighboring $B S$ transmits data to a $M T$ located in the outer area with more power during the same time slot and vice versa. The number of the time slots assigned to the inner or outer area is based on the user distribution of the two-cell system. How to choose the users to be scheduled in the inner and outer area can be determined by various performance criteria, such as channel condition, priority, revenue and throughput.

Within the simulation, we assume a two-cell system model as shown in Fig. 4.2. The distance between the base station and the edge of the outer area $(D)$ is $1000 \mathrm{~m}$. We do not count the users located in the area too close to the base station (the area is a square with side distance of $100 \mathrm{~m}$ ). We assume the mobile channels are affected by the log-distance path loss and log-normal shadowing where the corresponding parameters are $\alpha=4$ and the $\sigma=8 \mathrm{~dB}$. This model gives an attenuation which is the product of the fourth power of the distance and a log-normal random variable whose standard 
deviation is $8 \mathrm{~dB}$. That is, the path loss between the $M T$ and the $B S$ is proportional to $10^{(\xi / 10)} \ell^{-4}$, where $\ell$ is distance from $M T$ to the $B S$ and $\xi$ is a Gaussian random variable with standard deviation $\sigma=8 \mathrm{~dB}$ and zero mean. In the simulation, we assume that the channel conditions do not change within a time slot. The bandwidth $(W)$ of the TDDCDMA system is $1.25 \mathrm{MHz}$. We employ Binary Phase Shift Keying (BPSK) modulation technique in the transmissions. The relationship between the probability of bit error $\left(P_{e}\right)$ and the energy-per-bit to interference power density ration $\left(E_{b} / I\right)$ is obtained as [1]:

$$
P_{e}=\mathrm{Q}\left(\sqrt{\frac{2 E_{b}}{I}}\right) .
$$

That is, we assume the interference power is Gaussian-distributed in the above computation. In order to achieve the desired performance, that is $P_{e} \leq 10^{-3}$, we set the threshold $(\gamma)$ for $E_{b} / I$ as $5 \mathrm{~dB}$. The relationship between the number of the users $(N)$ and the density of the user distribution $\left(\rho_{a}, \rho_{b}\right)$ and the area of the cell is given in (5.18). The parameters in the simulation are summarized in Fig. 6.1.

\begin{tabular}{|c|c|}
\hline Parameter & Value \\
\hline System Bandwidth (W) & $1.25 \mathrm{MHz}$ \\
\hline $\begin{array}{l}\text { Threshold for } E_{b} / N_{o} \\
(\gamma)\end{array}$ & $5 \mathrm{~dB}$ \\
\hline$\alpha$ of path loss & 4 \\
\hline$\sigma$ of shadow fading & $8 \mathrm{~dB}$ \\
\hline Number of users ( N) & $20-200$ \\
\hline $\begin{array}{l}\text { Distance between the edge } \\
\text { of the outer area and BS ( D ) }\end{array}$ & $1000 \mathrm{~m}$ \\
\hline $\begin{array}{l}\text { Distance between the edge } \\
\text { of the inner area and } B S(d)\end{array}$ & $200-1000 \mathrm{~m}$ \\
\hline $\begin{array}{c}\text { Density of the inner area } \\
\left(\mathrm{P}_{\mathbf{a}}\right)\end{array}$ & $1.7 \times 10^{-5} \sim 6 \times 10^{-4}\left(\right.$ users $\left./ \mathrm{m}^{2}\right)$ \\
\hline $\begin{array}{l}\text { Relationship between } N \text { and } \\
\rho_{a} \text { and } P_{b}\end{array}$ & $N=4\left[P_{a} \times\left(d^{2}=10000\right)+P_{b} \times\left(D^{2}-d^{2}\right)\right]$ \\
\hline SIR & $8-90 \mathrm{~dB}$ \\
\hline \multirow{2}{*}{$\begin{array}{c}\text { Power adjustment factor } \\
(\varphi)\end{array}$} & for ULISS \\
\hline & $\varphi=(d / D)^{2}$ for CLDSS \\
\hline
\end{tabular}

Figure 6.1: Parameters Used in the Simulation.

In order to compare the average throughput of the CLDSS system with the ULISS 
scheme, we use the random selection of users in our simulation. Employing this selection criterion, during the period of transmissions to users in the inner area, we randomly choose a user from the inner area. We call this scheme as "CLDSS I". Similarly, we randomly choose a user in the outer area during the time slots in which $B S$ transmits signals to the users located in the outer area. On the other hand, we use a "Best Channel First" selection criterion in order to realize the full capacity of the CLDSS scheme for the system. In this scheme, we choose a user who has the best channel. We call this scheme as "CLDSS II".

In the simulation, the procedure to calculate the average throughput of the system is outlined in the flowchart in Fig. 6.2. During the transmissions,

1. the base station determines the number of time slots allocated to the users located in the inner and outer areas, which is based on the percentage of the users in the two areas. For example, if there are $20 \%$ users located in the inner area, the base station allocates $20 \%$ time slots to the inner area.

2. during this period, the base stations randomly choose the users in accordance with CLDSS I scheme or choose the users who have the best channel in accordance with CLDSS II scheme.

3. the base station sets the power adjustment factor $(\varphi)$ based on the division of the inner and outer areas. Then, the base station allocates the signal power level to the users. That is, the lower signal power is transmitted to the users located in the inner area and the higher signal power is transmitted to the users located in the outer area which are affected by the factor $\varphi$.

4. the base stations transmit signal to the certain users with allocated power level and scheduled time slots. 


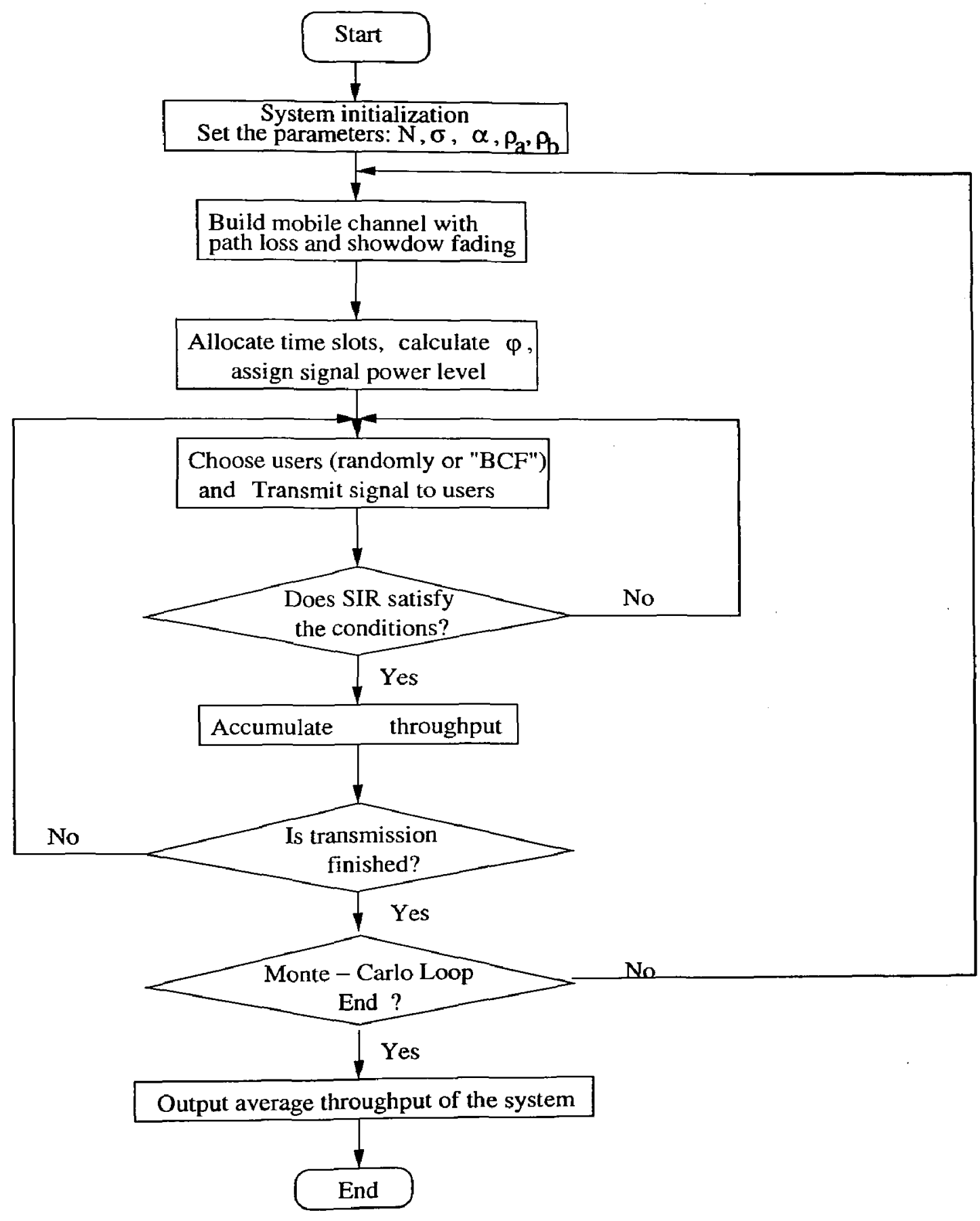

Figure 6.2: Flowchart of the Procedure in the Simulation. 


\subsection{Comparison of the CLDSS I Scheme with the ULISS scheme}

We analyzed the performance of the CLDSS I scheme in the previous chapter. By comparing the numerical results with the ULISS and CLISS schemes, we found that there are several advantages in the CLDSS schemes. In this section, we show some results from the simulation study comparing these scheduling algorithms. During the transmission, we randomly choose users in all three schemes.

\subsubsection{Effect of the Normalized Distance on the Throughput of System with Uniform User Distribution}

Fig. 6.3 shows the results of simulation using the CLDSS scheme, the ULISS scheme and the CLISS scheme, with uniform user distribution where the user density $\rho_{a}=\rho_{b}=$ $2.525 \times 10^{-5}$ (users $/ m^{2}$ ) and the number of users $N=100$. It shows us how the normalized distance of the inner area affects the throughput of the system. For clarity, Fig. 6.3 is shown in the range of $0.3<d^{*}<0.8$ in Fig. 6.4.

From these Figures, we can make the following observations:

- when the normalized distance $\left(d^{*}\right)$ of the inner area is less than about 0.33 , the performance of the CLDSS I scheme is the best.

- if $d^{*}>0.33$, the performance of the ULISS scheme is the best.

- There is a key point for the normalized distance, which is $d^{*}=0.33$ with uniform user distribution of users in the cell. The reasons were discussed in the chapter 5 .

From the figure, we can conclude that the simulated and analyzed results are in close agreement. 


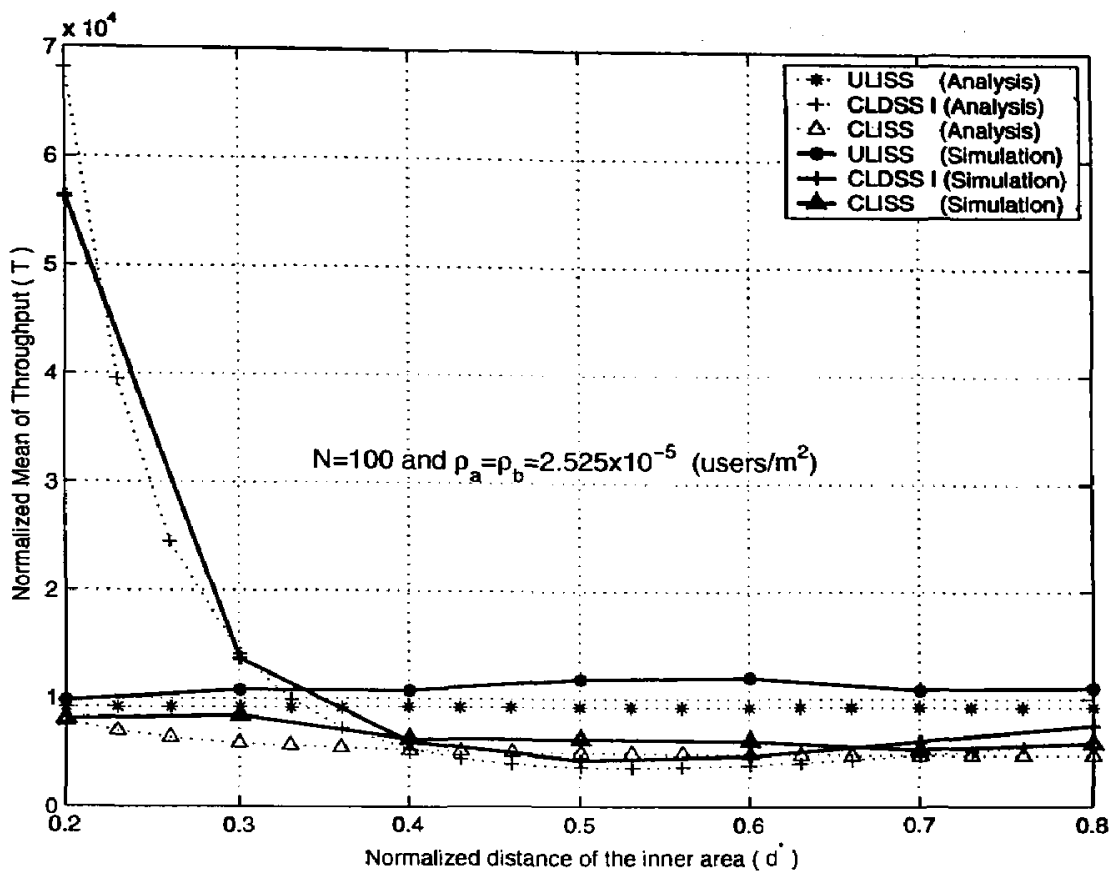

Figure 6.3: Throughput Affected by the Normalized Distance $\left(d^{*}\right)$ with Uniform User Distribution.

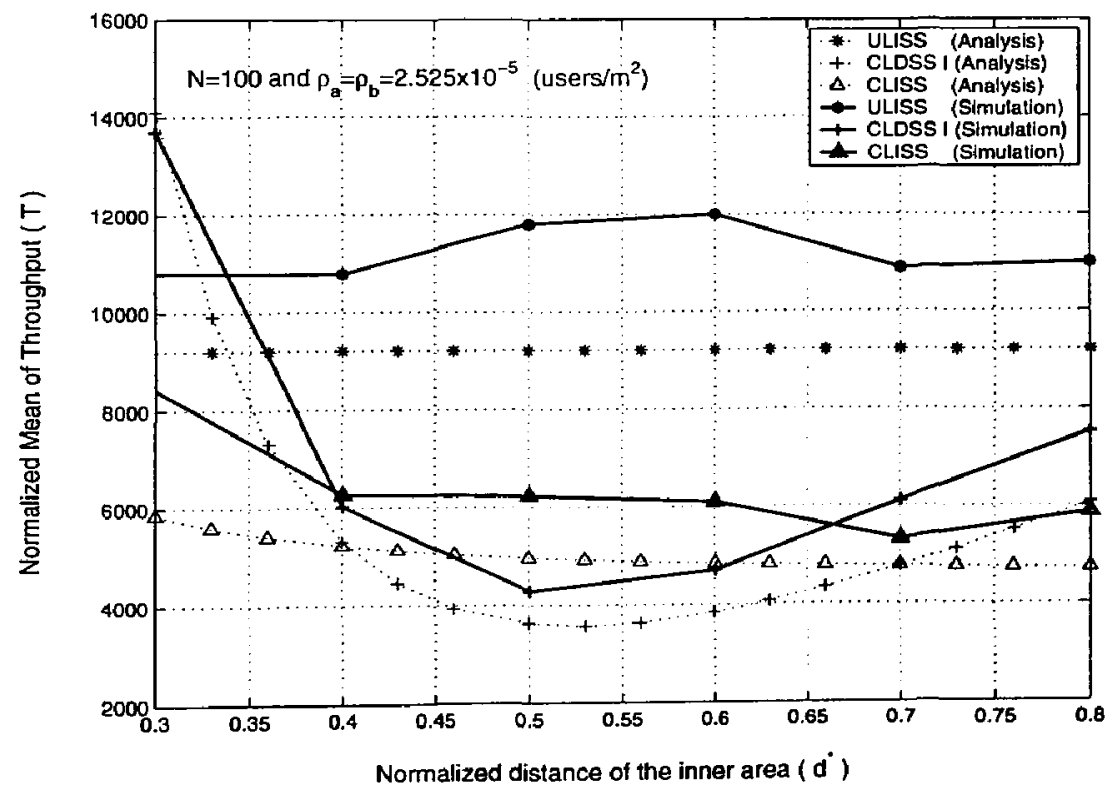

Figure 6.4: Throughput Affected by the Normalized Distance with Uniform User Distribution. $0.3<d^{*}<0.8$ 


\subsubsection{Effect of the Normalized Distance on the Throughput of System with Non-Uniform User Distribution}

Fig. 6.5 shows us the results of the throughput affected by the normalized distance $\left(d^{*}\right)$ with non-uniform user distribution.

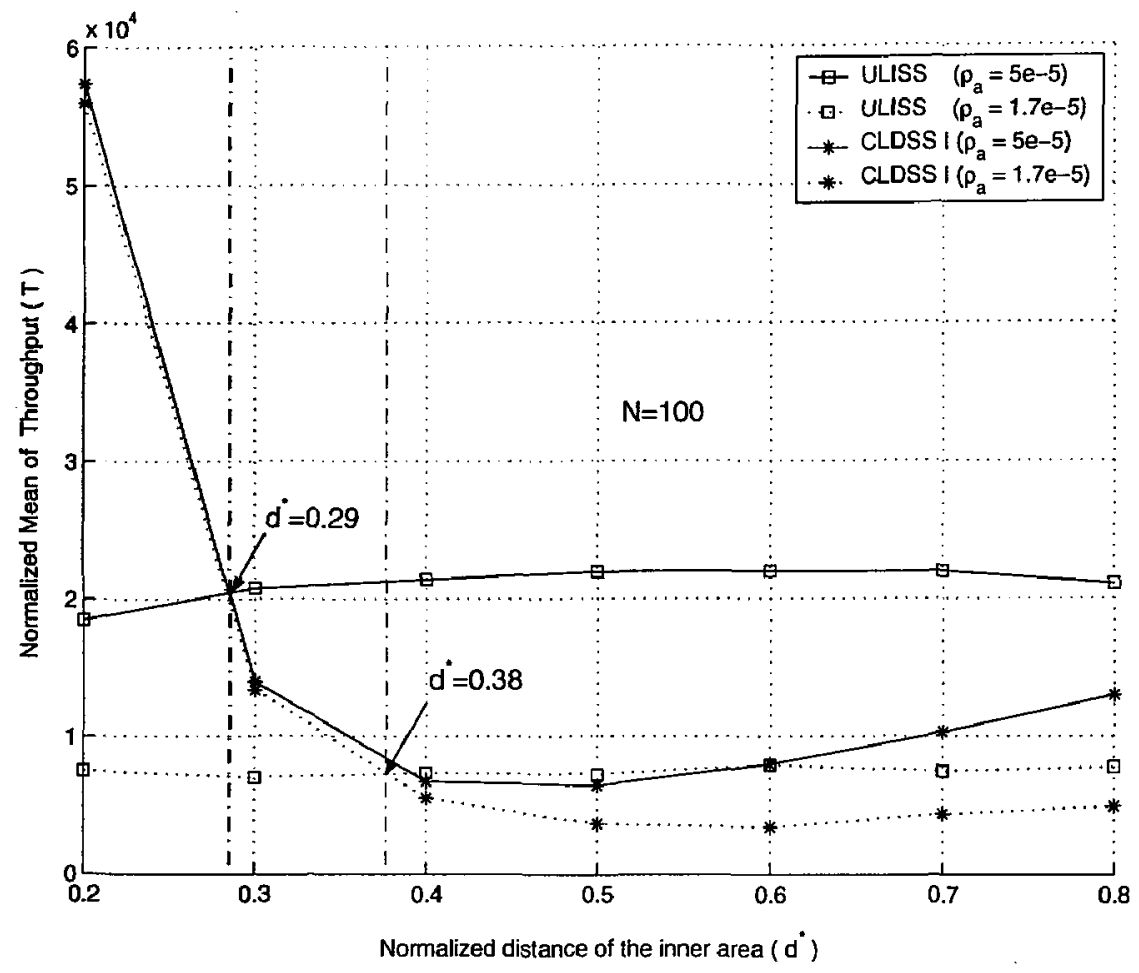

Figure 6.5: Throughput with Non-uniform User Distribution.

From Fig. 6.5, we can get:

- In the ULISS scheme, when the density $\rho_{a}$ decreases, the throughput of system reduces distinctly. However, the change of the average throughput with increasing inner user density in the CLISS I system is small.

- There is also a key point $\left(d^{*}=0.29\right)$; that is, when $d^{*}<0.29$, the performance of CLDSS I is better than ULISS for inner user density of $5 \times 10^{-5}$ (users $/ \mathrm{m}^{2}$ ); When the $d^{*}>0.29$, the performance of ULISS is better. Similarly, the key point for 
inner user density of $1.7 \times 10^{-5}$ (users $/ \mathrm{m}^{2}$ ) is 0.38 . In comparing with uniform user distribution system, the key point of $d^{*}$ is less when the users are non-uniformly distributed. The reasons are: (1) While $\rho_{a}$ increase, such as $\rho_{a}=5 \times 10^{-5}$ (users $/ \mathrm{m}^{2}$ ), more users are located in the inner area. Hence, the inner area can be allocated more time slots. (2) Because the base station allocates lower power to the users located in the inner area in the CLDSS system, the throughput from the inner area using CLDSS I is smaller than that using ULISS.

It can be noted that the key point obtained using simulated and analyzed results are also very close $\left(0.28\right.$ and 0.36 respectively for $\rho_{a}=5 \times 10^{-5}$ and $\rho_{a}=1.7 \times 10^{-5}$ (users $/ \mathrm{m}^{2}$ ) in the analysis).

\subsubsection{Effect of the User Density on the Throughput}

Fig. 6.6 shows us the effect of the user density on the throughput with non-uniform user distribution. It can be seen that the results from analysis and simulation are very close validating the theoretical study done in chapter 5 . When we set the number of the users to be 100 and the inner area normalized distance to be 0.2 , the performance of the CLDSS I and the ULISS can be shown in Fig. 6.6. It is clear that when $\rho_{a}<1.5 \times 10^{-4}$ (users $/ \mathrm{m}^{2}$ ) (or the percentage of users in the inner area is less than 18\%), the performance of the CLDSS I is better. Otherwise, when $\rho_{a}>1.5 \times 10^{-4}$ (users $/ \mathrm{m}^{2}$ ), the performance of the ULISS is better. The reasons are discussed before.

\subsubsection{Modification of the CLDSS}

Since there is a key point for the user density, for example, the percentage of users in the inner area is $18 \%$ when the number of users is 100 and the normalized distance is 0.2 . In order to increase the throughput, we can combine the CLDSS I with the ULISS. That is, if the percentage of the users in the inner area is less than $18 \%$, we employ the CLDSS I scheme and otherwise the ULISS scheme. Fig. 6.7 shows us the results of the modified CLDSS scheme with non-uniform user distribution based on the effect of the inner area 


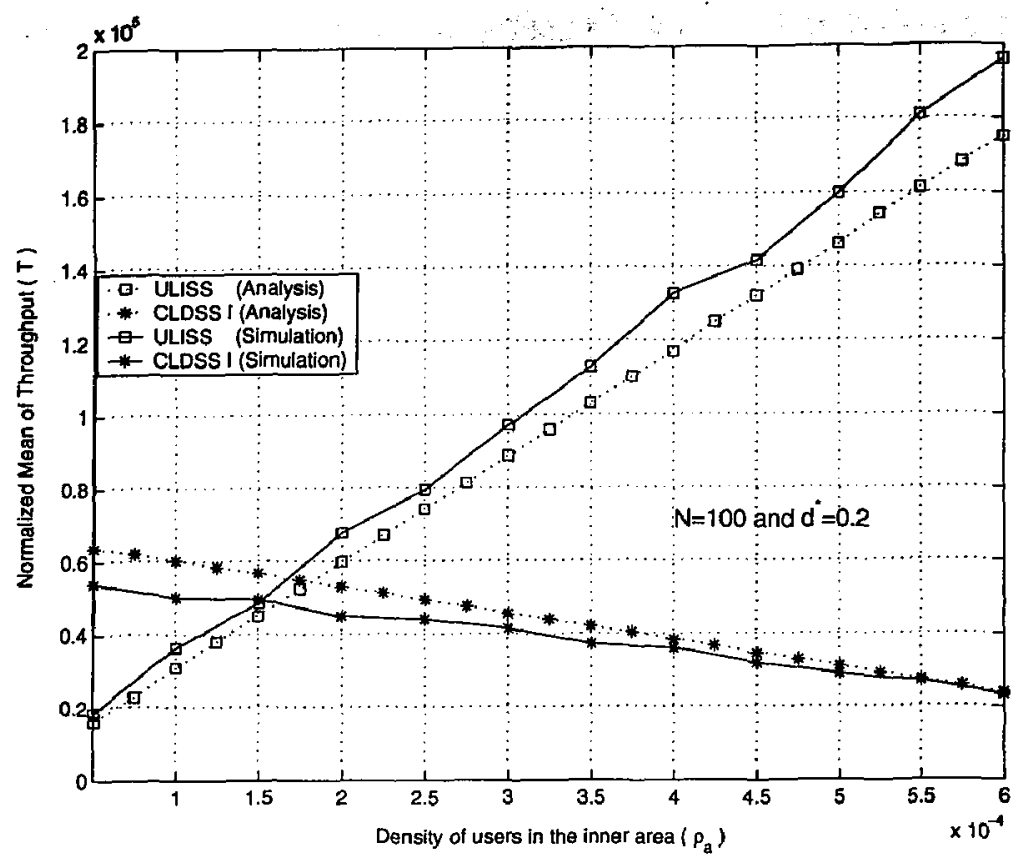

Figure 6.6: Throughput Affected by User Density.

user percentage on the throughput.

\subsubsection{Effect of the Number of Users on the Throughput}

Fig. 6.8 shows the results of the effect of the number of users on the throughput with nonuniform user distribution. From the result, one can see that the performance of proposed scheme is robust with the number of users in the cell. However, as expected, the ULISS scheme is lower not only in terms of throughput but also in terms of sensitivity to traffic in the cell.

\subsection{Comparison of CLDSS I Scheme with CLDSS II Scheme}

The motivation of the proposed scheduling scheme is to reduce the interference from the neighboring base stations in order to improve the downlink performance. By the method of allocating the radio signal power with different levels and coordinating transmissions 


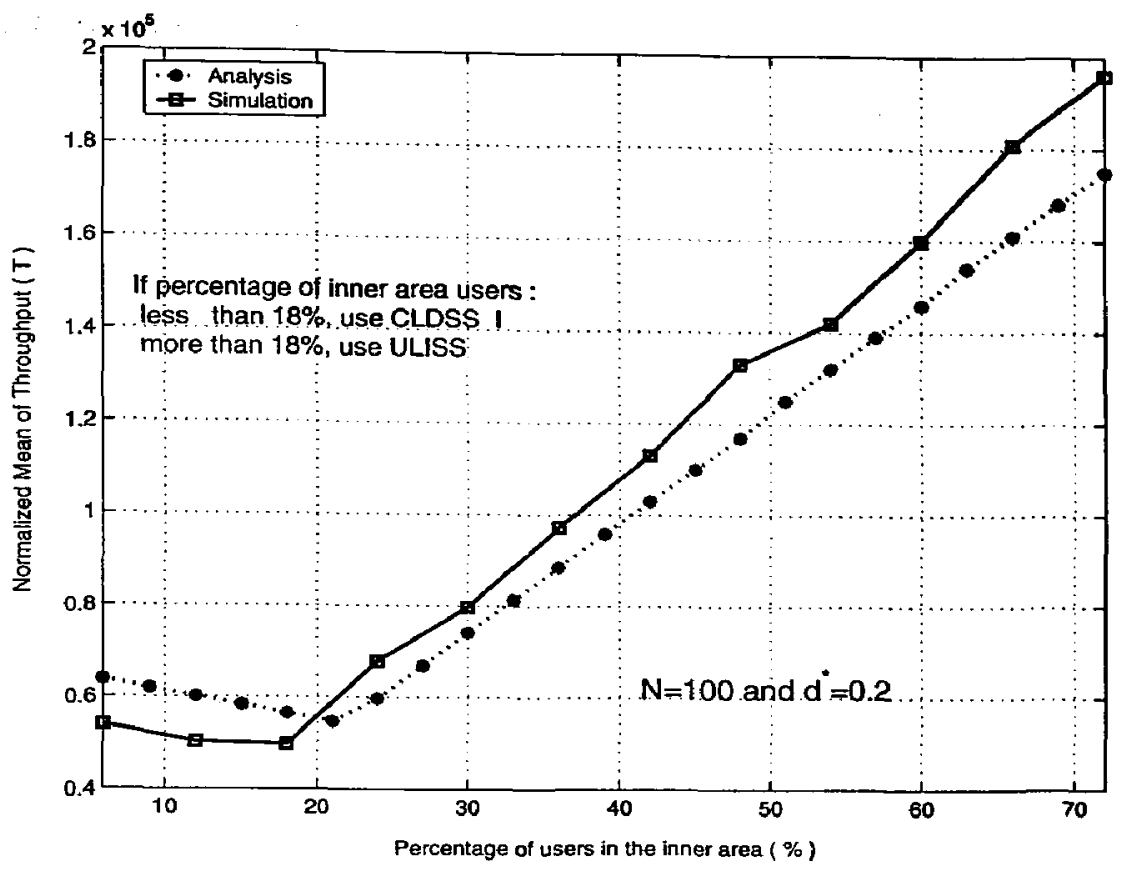

Figure 6.7: Throughput Affected by User Percentage.

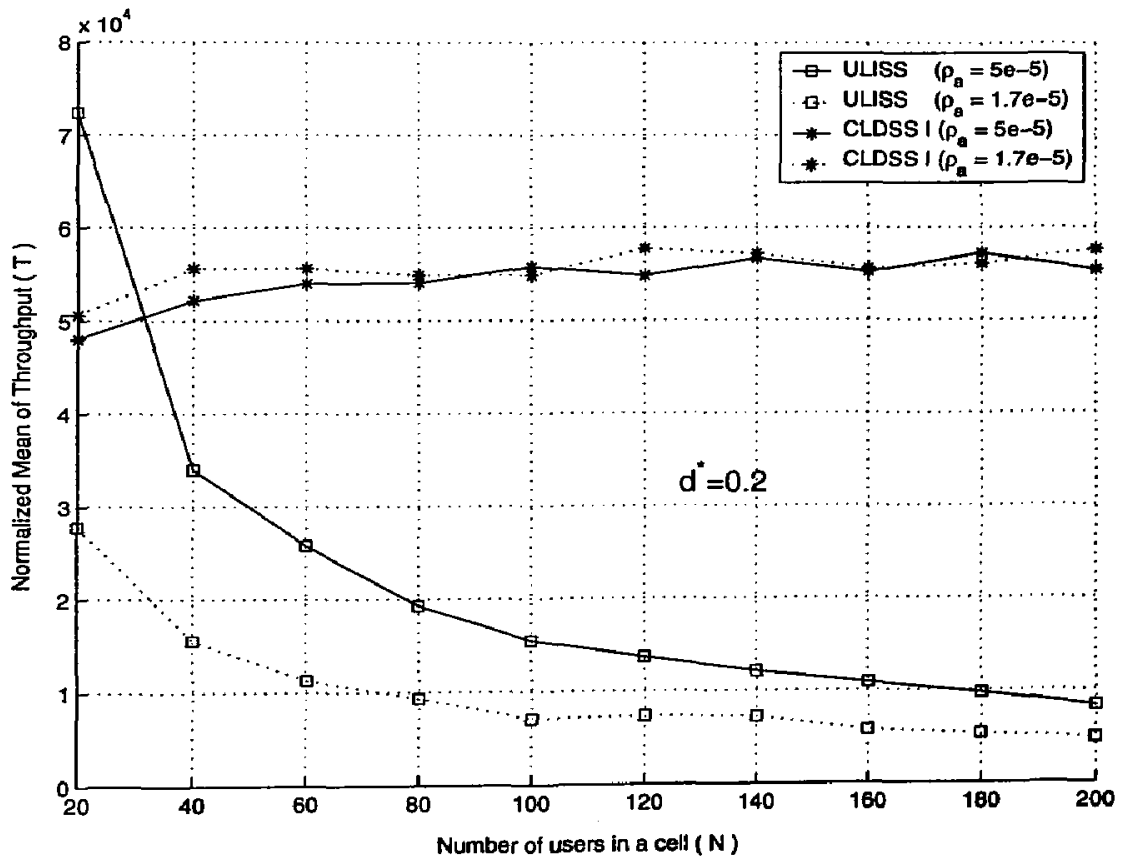

Figure 6.8: Throughput Affected by the Number of the Users in a Cell. 
with other cells, we can increase the throughput as well as the fairness. However, there is also some room to improve the performance by considering different ways to choose the users depending on different criteria. For example, we can choose the best channel in any time slot (CLDSS II) instead of randomly choosing any user in each of the inner and outer area (CLDSS I). Using CLDSS II, it is possible to achieve higher throughput in the system. In order to compare the performances of the two versions of the CLDSS scheme, the results of the simulation are shown next:

\subsubsection{Results with Uniform User Distribution}

Fig. 6.9 shows the results of the CLDSS schemes with uniform user distribution, where the number of users is 100 and density $\rho_{a}=\rho_{b}=2.525 \times 10^{-5}$ (users $/ m^{2}$ ). There are some features of the CLDSS II comparing with the CLDSS I:
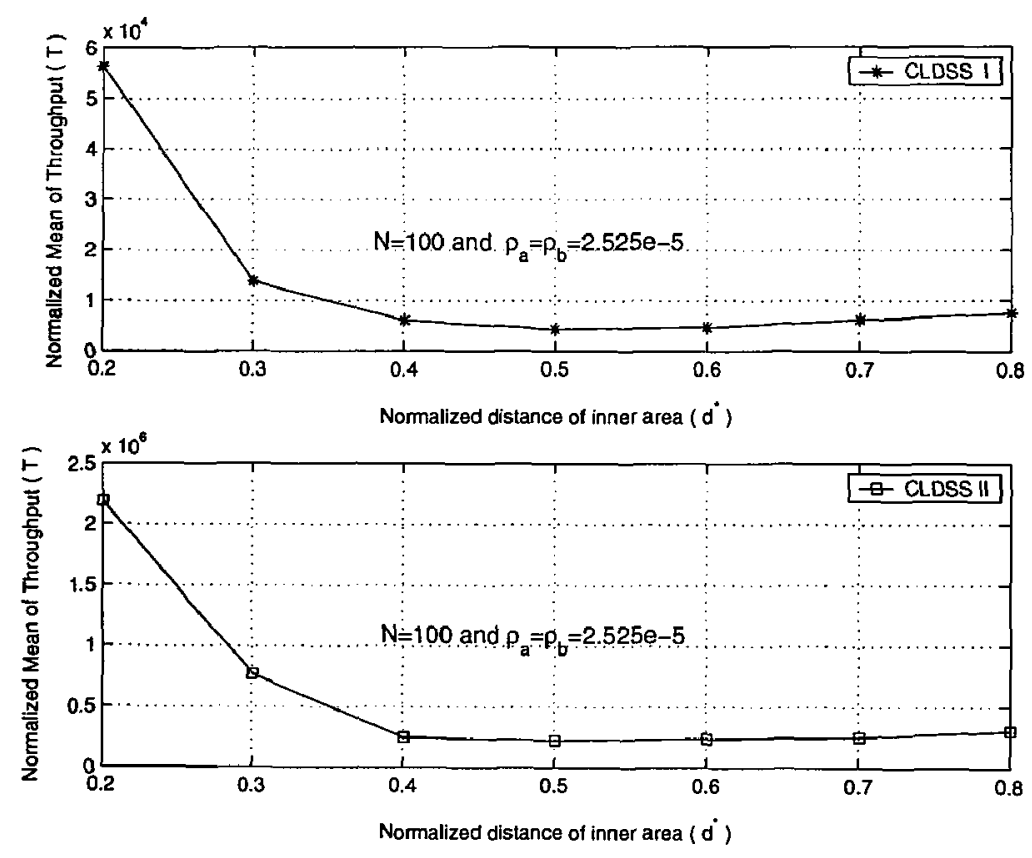

Figure 6.9: Throughput with Uniform User Distribution.

- The throughput of the CLDSS II is obviously higher than that of the CLDSS I. 
Because the CLDSS II scheme picks the best channel user to transmit data, it can achieve higher data rate.

- With the normalized distance $\left(d^{*}\right)$ reducing, the throughput of the system increases. The power adjustment factor $\varphi=(d / D)^{2}=\left(d^{*}\right)^{2}$, when $d^{*}$ reduces, $\varphi$ also reduces. That is, when base station transmits data to a user located near the border with full power $P$, the neighboring base station chooses a user located in its inner area with lower power $\varphi P$. Hence, the neighboring interference should be reduced, which is useful to improve the throughput. At this point, the two versions of the CLDSS scheme are similarly behaving in providing the throughput.

\subsubsection{Results for Non-Uniform User Distribution}

In the simulation, we set the number of users to be 100 . We can obtain the effect of the normalized distance $\left(d^{*}\right)$ with $\rho_{a}=5 \times 10^{-5}$ or $\rho_{a}=1.7 \times 10^{-5}$ (users $\left./ m^{2}\right)$ on the throughput, which are shown in Fig. 6.10.

- Similar to the uniform user distribution case discussed above, the CLDSS II can achieve higher throughput.

- When the normalized distance $\left(d^{*}\right)$ is larger than 0.5 , the throughput of the CLDSS II with $\rho_{a}=5 \times 10^{-5}$ (users $/ m^{2}$ ) is higher than that of the CLDSS II with $\rho_{a}=$ $1.7 \times 10^{-5}$ (users $/ \mathrm{m}^{2}$ ). This can be explained with the following reasons: (1) when $d^{*} \geq 0.5$, the power adjustment factor $\left(\varphi=\left(d^{*}\right)^{2}=(d / D)^{2}\right)$ is less than 0.025 , so the compensation from the users located in the outer area is reduced; (2) when $\rho_{a}=5 \times 10^{-5}$ (users $/ \mathrm{m}^{2}$ ), more users are located in the inner area compared with when $\rho_{a}=1.7 \times 10^{-5}$ (users $\left./ m^{2}\right)$. As a result, the inner area users can get more time slots. 

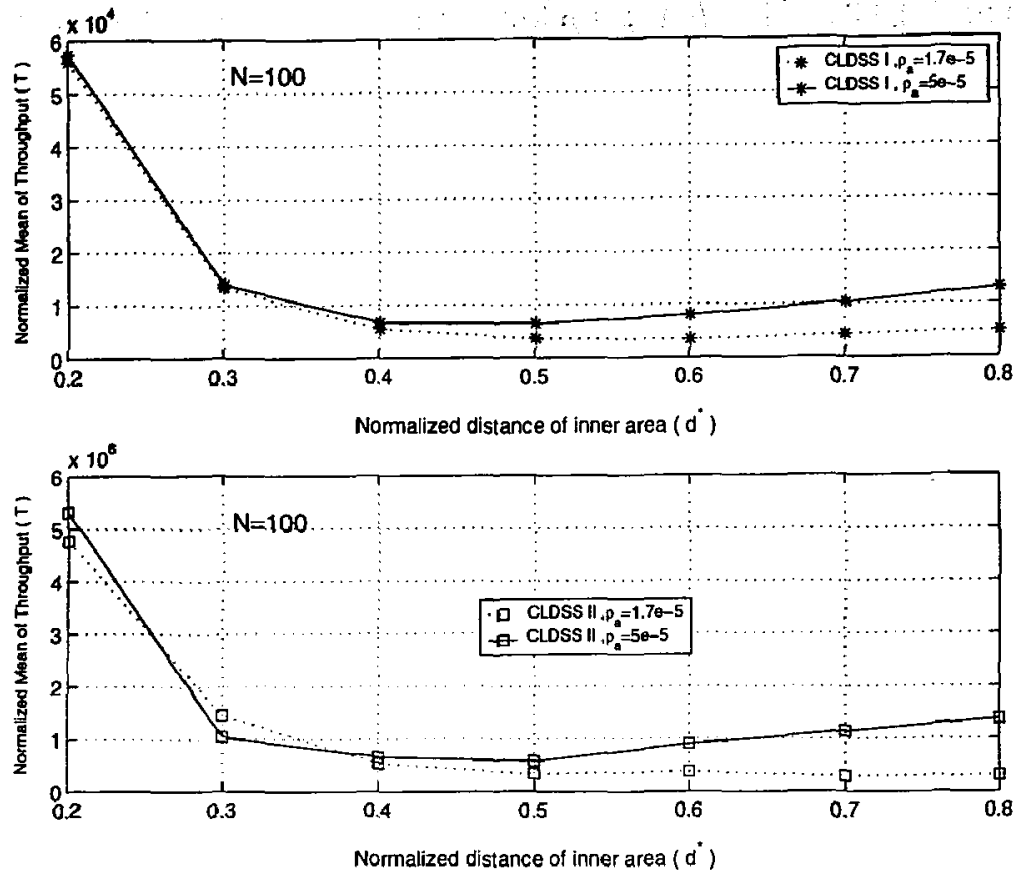

Figure 6.10: Throughput with Non-uniform User Distribution.

\subsubsection{Effect of User Density on the Throughput}

The effect of user density on both of the two versions of the CLDSS scheme is similar as shown in Fig. 6.11, where the number of users is 100 and the normalized distance is 0.2. When $\rho_{a}$ increases, there are less users located in the outer area. On one hand, in general, because the distance between the base station and the users located in the outer area is larger, the channel condition is weaker. On the other hand, the base station allocates lower signal power to users located in the inner area, in other words, the neighboring interference is reduced. If the factor $\varphi$ is small enough, such as $\varphi=0.04$, the signalto-noise ratio for users located in the outer area is increased. The throughput can be compensated by the users located in the outer area. But, with the number of users in the outer area reducing, the time slots allocated to the outer area is reduced. As a result, the compensation of throughput from the outer area is reduced, so that the throughput of the system is reduced as shown in Fig. 6.11. 

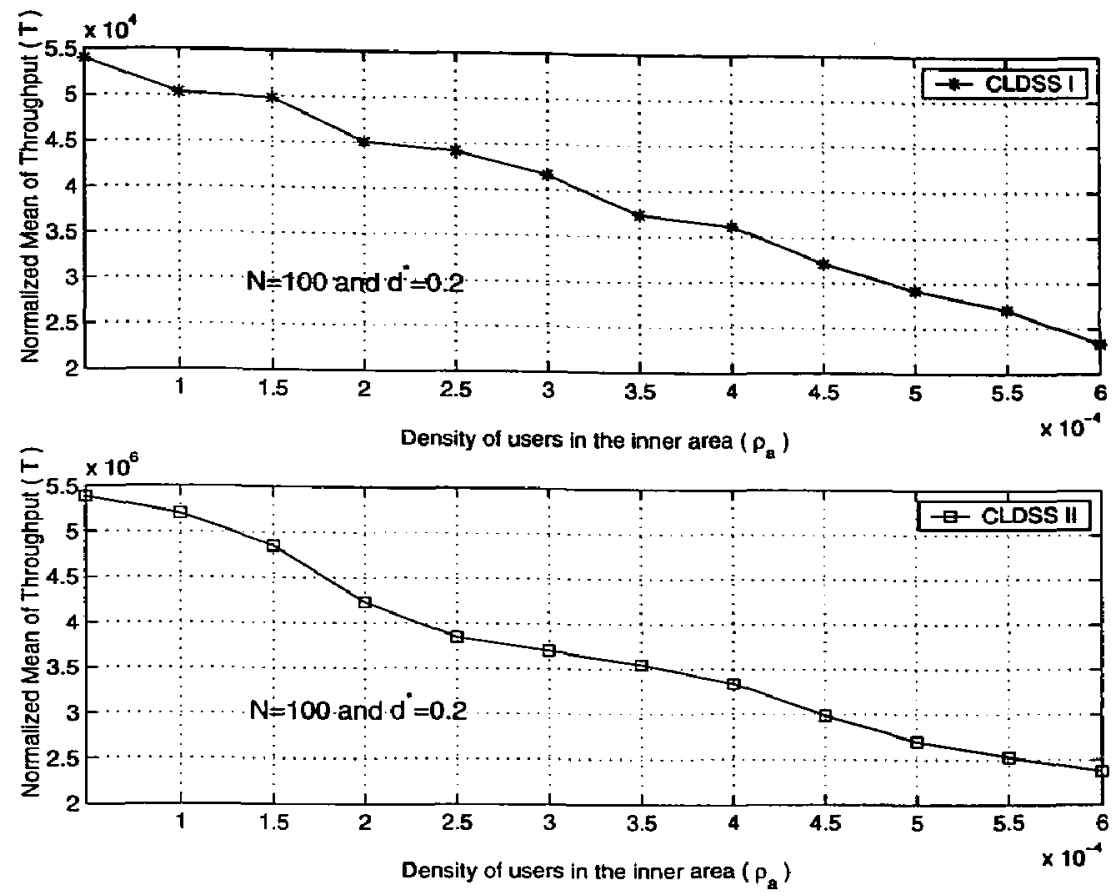

Figure 6.11: The Effect of User Density on the Throughput.
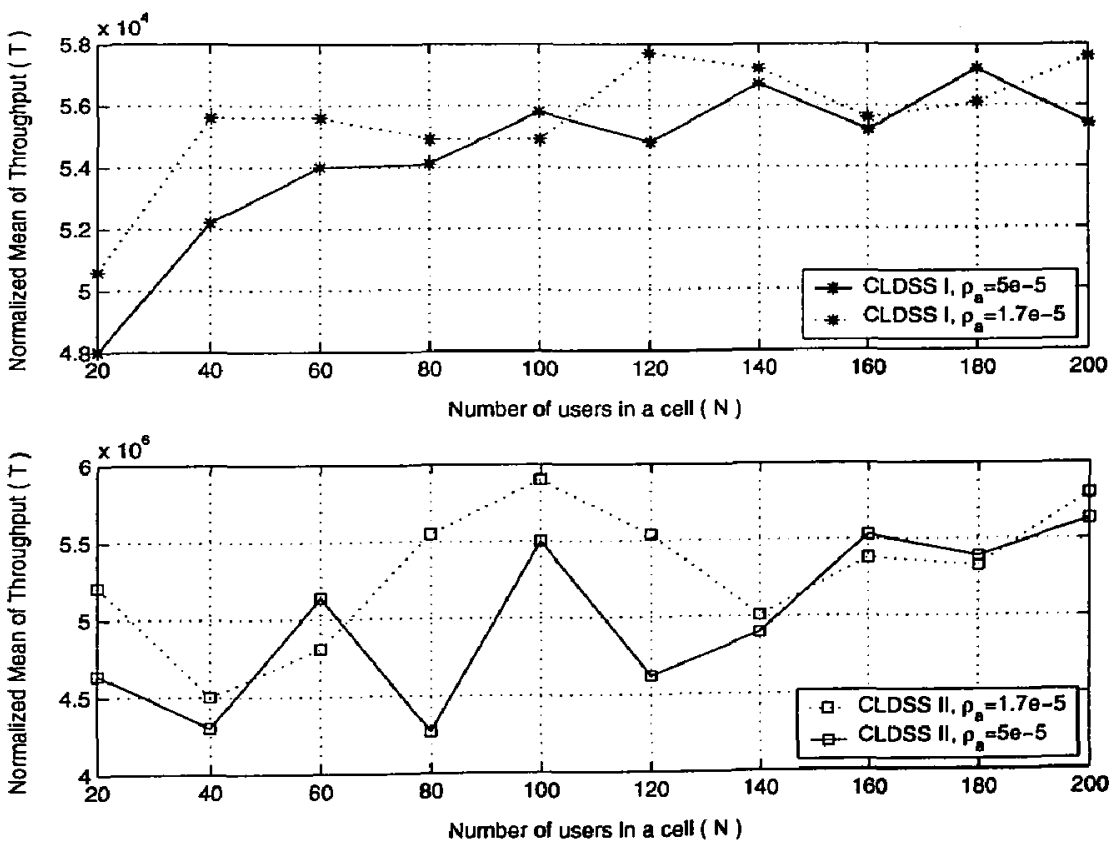

Figure 6.12: The Effect of User Number on the Throughput with the Non-uniform User Distribution. 


\subsubsection{Effect of User Number on the Throughput}

The effect of the number of the users on the throughput with non-uniform user distribution is not significant, because the base stations just chooses one user, either using random selection in CLDSS I or choose a user who has the best channel in CLDSS II during any time slot. The feature of the CLDSS scheme makes the system to have "soft throughput", that is, the CLDSS scheme is not very sensitive to traffic in the cell as shown in Fig. 6.12.

\subsection{Summary}

In this chapter, we presented the results from the simulation studies. Simulation results showed the consistency with the theoretical analysis. In the meantime, simulation study also demonstrated that the proposed scheme can limit the interference from the neighboring base stations and improve the performance of the CDMA system. When we set the normalized distance $d^{*}$ and the user density of inner area to be less than the key points, the throughput of the system using the CLDSS scheme was better the the ULISS scheme. Otherwise, the ULISS scheme can achieve higher throughput. Therefore, we modified the CLDSS scheme as: if $d^{*}$ and $\rho_{a}$ ware less than the key point, we used the original CLDSS scheme; if $d^{*}$ and $\rho_{a}$ was larger than the key point, we used the ULISS scheme in order to achieve higher throughput. In the meantime, the CLDSS scheme can improve the fairness for non-uniform user distribution system and make the CDMA to have "soft throughput". The relationship between the key points, the normalized distance $\left(d^{*}\right)$ and the density of users in the inner area $\left(\rho_{a}\right)$ can be shown in Fig. 6.13, where the number of users is 100 .

On the other hand, when we employed Best Channel First (BCF) based user selection in our CLDSS scheme, the throughput of the system increased significantly. However, using the BCF selection, some users with weak channel conditions can not get the data rate from the base station which reduces the fairness, although we alternatively choosed the users from the inner and outer area based on the percentage of time slots. 


\begin{tabular}{|c|c|c|}
\hline$\rho_{\mathrm{a}}\left(\right.$ users $\left./ \mathrm{m}^{2}\right)$ & $\begin{array}{c}\text { Percentage of users } \\
\text { in the inner area } \%)\end{array}$ & Key Point $\left(\mathrm{d}^{*}\right)$ \\
\hline $1.7 \times 10^{-5}$ & $8 \%$ & 0.36 \\
\hline $2.525 \times 10^{-5}$ & $10 \%$ & 0.33 \\
\hline $3.5 \times 10^{-5}$ & $12 \%$ & 0.31 \\
\hline $5 \times 10^{-5}$ & $14 \%$ & 0.28 \\
\hline $6 \times 10^{-5}$ & $15 \%$ & 0.27 \\
\hline
\end{tabular}

\begin{tabular}{|c|c|c|c|}
\hline \multirow{2}{*}{$\mathrm{d}^{*}$} & \multicolumn{2}{|c|}{ Percentage of users } & Key Point \\
\hline & in the inner area & $(\%)$ & $\rho_{\mathrm{a}} \quad\left(\right.$ users $\left./ \mathrm{m}^{2}\right)$ \\
\hline 0.2 & $18 \%$ & & $1.5 \times 10^{-4}$ \\
\hline
\end{tabular}

Figure 6.13: The Relationship between the Key Points, Normalized Distance $\left(d^{*}\right)$ and the Density of Users in the Inner Area $\left(\rho_{a}\right)$. 


\section{Chapter 7}

\section{Summary}

\subsection{Conclusion}

With the development of the digital communication techniques, especially, popularization of the Internet service, the requirements for high speed data services are rising rapidly. Currently, the wireline Internet services can provide the data rate more than 2Mbps. More and more wireless subscribers demand the same quality-of-service (QoS) of high speed data service as the wireline communication system. Hence, 3G communication standards have been proposed and 3G system are in deployment in order to meet the requirements of the high data rates. $3 \mathrm{G}$ systems promise multi-maga bit Internet access, communications using Voice over Internet Protocol, increased network capacity.

Because the characteristics of the high speed data communication are fundamentally different from voice communication, notably, (i) throughput required by the downlink is much higher than that of uplink transmission, (ii) most of the high speed data services can tolerate some delay and data traffic is bursty, and (iii) data rate requirements are very high. On the other hand, the mobile channel conditions vary with the mobile environment timely. Therefore, the relative delay tolerance of data applications, together with bursty traffic characteristics, open up the possibility for scheduling transmissions so as to optimize throughput by exploiting the variation in the channel conditions. TDD-CDMA scheme is one of the schemes adopted in the 3G communication system. TDD-CDMA combines the TDMA and CDMA technologies which inherit their own advantages, such as, the high 
intra-cell capacity of TDMA and the inter-cell interference suppression ability of CDMA. Moreover, we can employ some scheduling algorithms in the TDD-CDMA system to improve the performance of the high speed data services based on several performance criteria.

The HDR scheduling algorithm was designed for the downlink transmission within which the system schedules transmission to a mobile user that has the strongest signal levels. The HDR algorithm satisfies fairness property known as proportional fairness and was adopted in the $3 \mathrm{G}$ variation by some standards. However, there are some disadvantages. For example, the use of maximum power increases the interference to users in the neighboring cell (especially, the users located near the border). Meanwhile, transmitting data to the users which are located closer to base station with full transmission power can usually get much higher data rate than the maximum rate of the system. Hence, there are some HDR-like algorithms proposed in order to achieve different kind of performance in accordance with the different criteria, such as priority, channel condition or maximum achievable rate. The "HDR-like" scheduling algorithms are based on the original HDR scheme proposed by Qualcomm with the feature that, in any time slot, only a subset of users are scheduled for transmission based on some criteria.

For the downlink transmission, the major interference comes from the neighboring base stations. Especially, when users are located near the border of the cells, they receive stronger interference power. Currently, to our knowledge, there is not a HDR-like scheduling algorithm which combines the coordination of transmissions and the power allocation algorithms for downlink transmission in the cellular communication system. Hence, we proposed a new scheduling scheme-Coordinated Location-based Downlink Scheduling Scheme (CLDSS). The motivation of the proposed scheme is to exploit the time varying channel and to limit the interference from the neighboring base stations. In the CLDSS scheme, the system chooses one user to be transmitted at one time slot in one cell. A cell is divided into two flexible co-centric areas based on the traffic load in the cell. We involve a power adjustment factor which is a function of the locations of the two areas, 
as $\varphi=(d / D)^{2}$. The base station allocates full power $(P)$ to the users located in the outer area, as well, allocates lower power $(\varphi P)$ to the users located in the inner area. The transmissions are coordinated with the transmissions in the neighborhood. That is, when a base station transmits data to a user that is located in the inner area, the neighboring base station has to choose a user who is located in the outer area of its home cell and vice versa. The system assigns the number of time slots to the users in the inner and outer areas based on the user distribution. In other words, if there are more users located in the inner area, then the system allocates more time slots to the inner area. Since the CLDSS scheme can control the allocation of the radio signal power and coordinate the transmissions between cells, the scheme is useful to restrain the interference. When we transmit data to the user in the inner area, generally, with better channel condition, we use lower signal power to reduce the co-channel interference to the user of the neighboring cells. On the other hand, when we transmit data to the user located in the outer area with full power, the neighboring base station chooses the user located in its inner area and transmits data with lower signal power in accordance with the coordinated scheme. Therefore, the neighboring interference is lower, which is useful to improve the signalto-noise ratio. As a result. the data rate for the user located in the outer area can be compensated.

From the results of the numerical analysis and simulation studies, we showed that the CLDSS scheme can increase the throughput of the system when we set the normalized distance of the inner area $\left(d^{*}\right)$ to be less than some "key point". And the CLDSS scheme can improve the fairness and make the system to have "soft throughput", especially, when more users are located near the border of the cells. In addition, when we choose the users in the two areas with different methods based on different performance criteria, the CLDSS scheme can meet the different requirements, such as, (1) Random selection-which is useful to improve the fairness for the users in the cell and the control is simple; (2) Best-Channel-First selection- which is useful to improve the throughput of the system. 


\subsection{Future Work}

Since the CLDSS scheme can coordinate the transmissions in the cellular system, it can improve the performance of the system. However, there is room to modify the proposed CLDSS scheme, because the original CLDSS scheme just considered two-cell system both in analysis and simulation which is relatively simple. In the future, we plan to consummate the CLDSS scheme from two facets: one is to increase the levels of co-centric areas; the other one is to increase the number of cells to mate it a real cellular system.

Similarly, the cell is divided by several co-concentric areas based on the traffic load of the system. The future model of the modified CLDSS scheme is shown as Fig. 7.1.

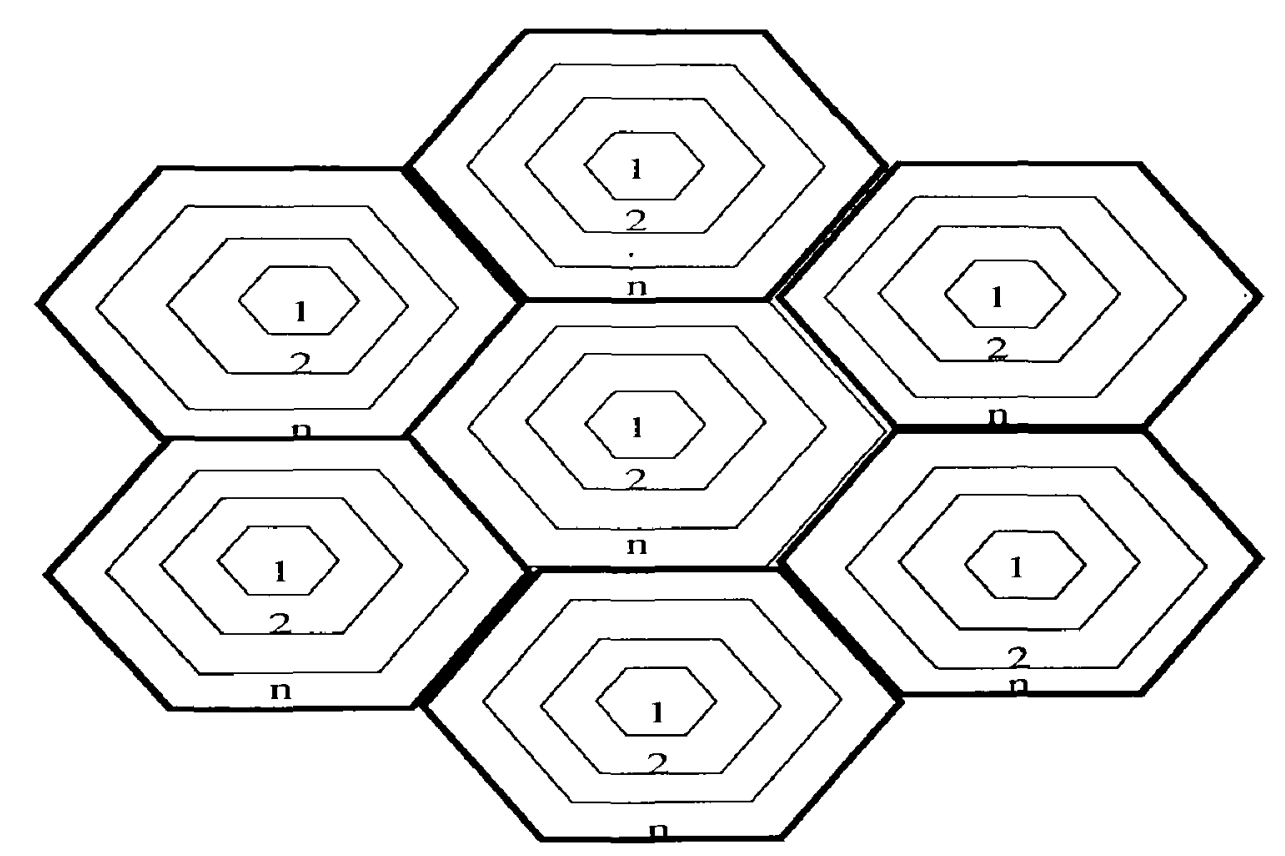

Figure 7.1: The Model for the Future Work.

We create a power adjustment factor to build up a pool of radio signal powers. The power adjustment $\varphi$ can be set as:

$$
\varphi=\left(\frac{d_{i}}{D}\right)^{m}
$$

where $i$ is the level of co-centric area, i.e., $i=1,2, \ldots, n . d_{i}$ is the distance between the edge of the $i$ level of co-centric area and the base station. $D$ is the distance between the 
edge of the cell and the base station. Because $\varphi$ can not be too small, otherwise, the radio signal power with $\varphi P$ is too small to transmit to data. Hence, we set $1 \leq m \leq 2$.

After a base station chooses a user, it will choose a radio signal power from the power pool to transmit data based on the location of the user. In the meantime, the cells of the system will coordinate their transmissions. In general, when a base station transmits data to a user located near the border of cells, the neighboring base station should avoid to transmit any data to any user who is located near the other scheduled user. 


\section{Bibliography}

[1] T. S. Rappaort, Wireless communications: principles and practice, Prentice-Hall, 1996.

[2] Giridhar Mandyam and Jersey Lai, Third-Generation CDMA Systems for Enhanced Data Services, Academic Press, 2002.

[3] Riaz Esmailzadeh and Masap Nakagawa, TDD-CDMA for wireless communications, Artech House, 2003.

[4] John G. Proakis, Digital Communications, McGraw Hill, 2001.

[5] Alagan Anpalagan and E. Sousa, "A distributed packet scheduling scheme with interference avoidance for non-real time applications in CDMA networks," (preprint), International Journal of Wireless Information Networks, Kluwer Academic Publishers, vol. 11, no. 2, pp. 79-92, April 2004.

[6] Paul Bender, Peter Black, Mattew Grob, Roberto Padovani, Nagabhushana Sindhuushayana, and Andrew Vlterbi, "CDMA/HDR: A bandwidth-efficient high-speed wireless data service for nomadic users," IEEE Commun. Mag., pp. 70-77, July 2000.

[7] A. Jalali, R. Padovani, and R. Pankaj, "Data throughput of CDMA-HDR a high efficiency-high data rate personal communication wireless system," IEEE Trans. Veh. Technol., vol. 3, pp. 1854-1858, 2000.

[8] F. Kelly, "Charging and rate control for elastic traffic," European Transactions on Telecommunications, vol. 8, pp. 33-37, 1997. 
[9] Mehmet Yavuz, David Paranchych, and Geng Wu, "Performance improvement of the HDR system due to hybird ARQ," IEEE Trans. Veh. Technol., vol. 3, pp. 1937-1941, 2001.

[10] Sarath Kumar and Sanjiv Nanda, "High data packets communications for cellular networks using CDMA: algorithms and performance," IEEE Journal on Selected Areas in Communications, vol.17, pp.472-492, March 1999.

[11] Hyun Chekal and Youngnam Han, "An efficient power allocation scheme in HDR systems," IEEE Trans. Veh. Technol., vol. 4, pp. 2197-2201, Oct. 2001.

[12] Laurence Mailaender, Howard Huang, and Harish Viswanathan, "Simple inter-cell coordination schemes for a high speed CDMA packet downlink," IEEE Trans. Veh. Technol., vol. 3, pp. 1845-1848, 2000.

[13] Gang Wu, Alagan Anpalagan, "A coordinated location-based downlink scheduling scheme (CLDSS) in a cellular CDMA network with partitioned cells," Electrical and Computer Engineering, IEEE CCECE 2004, Canadian Conference, pp. 0415-0419, May 2004.

[14] A. Bedekar, S.C. Borst, K. Ramanan, P.A. Whiting, and E.M. Yeh, "Downlink scheduling in CDMA data networks," Lucent Technologies, Technical Memorandum, 1999.

[15] Sem Borst and Phi Whiting, "Dynamic channel-sensitive scheduling algorithms for wireless data throughput optimization," IEEE Trans. Veh. Technol., vol. 52, no. 3, pp. 569-586, May 2003.

[16] Fredrik Berggren, Seong-Lyun Kim, Riku Jantti, and Jens Zander, "Joint power control and intracell scheduling of DS-CDMA nonreal time data," IEEE Journal on Selected Areas in Communications, vol. 19, no. 10, pp. 1860-1870, Oct. 2001. 
[17] Xin Liu, Edwin K. P. Chong, and Ness B. Shroff, "Opportunistic transmission scheuling with resource-sharing constraints in wireless networks," IEEE Journal on Selected Areas in Communications, vol. 19, no. 10, pp. 2053-2064, Oct. 2001.

[18] Songwu Lu, Vaduvur Bharghavan, and R. Srikant, "Fair scheduling in wireless packet networks," IEEE/ACM Transactions on Networking, vol. 7, no. 4, pp. 473-489, Aug. 1999.

[19] Peter Marbach and Randall Berry, "Downlink resource allocation and pricing for wireless networks," INFOCOM 2002. Twenty-First Annual Joint Conference of the IEEE Computer and Communications Societies, vol. 3, pp. 1470-1479, June 2002.

[20] Ramjee Prasad, Werner Mohr, and Walter Konh Auser, Third Generation Mobile Communication Systems, Artech House, 2000. 
$76: 7126$ 